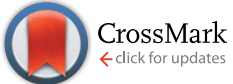

Cite this: RSC Adv., 2015, 5, 89698

Received 28th August 2015

Accepted 13th October 2015

DOI: $10.1039 / c 5 r a 17468 f$

www.rsc.org/advances

\section{Challenges and developments of self-assembled monolayers and polymer brushes as a green lubrication solution for tribological applications}

\begin{abstract}
Simon Watson, ${ }^{* a}$ Mengyan Nie, ${ }^{a}$ Ling Wang $^{a}$ and Keith Stokes ${ }^{\text {ab }}$
Self-assembled monolayers (SAMS), after originally being investigated due to their functions in changing surface wettability, have been significantly developed over the years. Many types of SAMs have been developed on a variety of substrates. However their formation mechanism, rate and quality are found to be influenced by many factors. A range of SAMs including single- and multi-component are included in this review with focus on the nano and macro tribological properties. More recently, surface initiated polymer brushes, i.e. macromolecular assemblies attached to a substrate, have emerged to be an alternative and promising method for surface modification. The ability to tether these macromolecules to tribological contacts is key to their resistance to shear under loaded contacts. This review also covers atom transfer radical polymerisation (ATRP) and the role of this technique in developing new lubrication solutions. Particular care has been taken to include the development of lubrication solutions for silicon nitride due to the importance of this material as an engineering ceramic. This paper reviews the stateof-the-art development of SAMs and polymer brushes especially the potential opportunities and challenges in applying them in tribological contacts as a lubrication solution.
\end{abstract}

\section{Introduction}

Whenever two surfaces come into contact there will be energy wasted due to friction which will result in wear or degradation of the surfaces. In the privately owned passenger vehicle market

${ }^{a}$ National Centre for Advanced Tribology at Southampton (nCATS), University of Southampton, Southampton SO17 1BJ, UK. E-mail: s.watson@soton.ac.uk

${ }^{b}$ Platform Systems Division, Dstl, Porton Down, Salisbury, Wiltshire SP4 OJQ, UK as well as heavy goods vehicles and busses, Holmberg et al. state that a third of the energy from fuel is used to overcome friction. ${ }^{1,2}$ Significant areas of friction are from such components as the drive chain, engine and transmission, tires and brakes. One way to reduce fuel expenditure on friction is to utilise correct and efficient lubrication solutions. Current liquid lubrication solutions are generally in two classes; organomolybdenum compounds and organic friction modifiers. ${ }^{3,4}$ Organic friction modifiers include carboxylic acids/free fatty

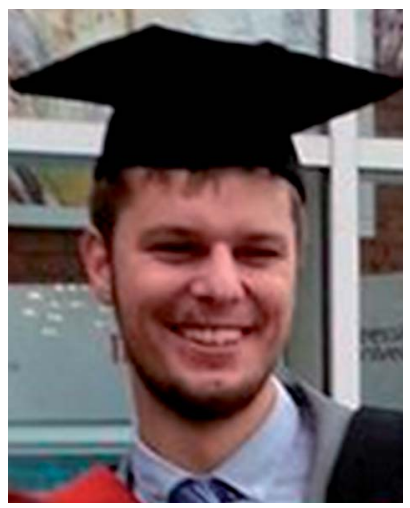

Simon Watson graduated with a degree in Chemistry from the University of Teesside. He also completed a placement at the Centre for Process Innovation with focus on printable electronics. He joined Southampton University as a postgraduate researcher in 2014 under the supervision of Ling Wang, investigating green lubrication solutions for silicon nitride hybrid contacts.

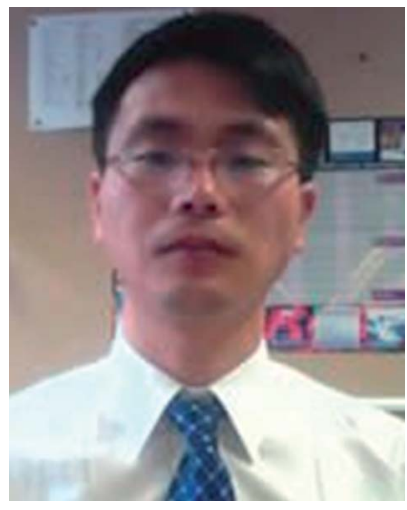

Mengyan Nie is currently working as a Research Fellow at the National Centre for Advanced Tribology at Southampton (nCATS), University of Southampton, UK. He received his BSc and MSc degrees in Chemistry from Lanzhou University, and PhD in Chromatography from Dalian Institute of Chemical Physics, Chinese Academy of Sciences, China. His research interests include microfluidics and microsystems for condition monitoring, electrochemical sensing techniques, corrosion management, and surface engineering. 
acids, alcohols, esters and amines. ${ }^{4}$ There are two accepted mechanisms that explain the mechanism of lubrication for organic friction modifiers. One mechanism is that the polar functional groups of the friction modifier adsorb onto the surface, much like self-assembled monolayers (SAMs), and the carbon chains form a barrier to prevent substrate-substrate adhesion thus lowering friction. ${ }^{3,5,6}$ The other model is semiordered viscous multilayers prevent contact. ${ }^{3,5}$ Free fatty acids are still used today to reduce friction, this is due to their ability to create closely packed monolayers. ${ }^{5,8}$ Long chain amines have also been reported in clutch systems, lubricated sliding contacts and MEMS devices., ${ }^{3,9,10}$ Molybdenum dithiocarbamates (MoDTC) have been introduced into engine oils since the 1950s as an antiwear additive, however, it was not until the 1970s where its application as a friction modifier was realised. ${ }^{3,11}$ MoDTC has been reported to form $\mathrm{MoO}_{3}$ resulting in high wear rates as $\mathrm{MoO}_{3}$ is abrasive. ${ }^{\mathbf{4 , 1 2}}$ The decomposition of MoDTC produces $\mathrm{MoS}_{2}$ sheets that bond onto surface asperities therefore reducing friction., ${ }^{\mathbf{4 1 3 - 1 6}}$ The role of zinc dialkyldithiophosphate (ZDDP) and MoDTC has been reported as the additives are shown to synergize well., ${ }^{3,416}$

However, in the current economic climate with the ever increasing costs of purchasing new equipment there is a greater need for more effective lubricants. When combined with the fact that in more recent years there are new limits on the amount of sulphur and phosphorus that can be used in lubricating oils. ${ }^{17,18}$ The removal of sulphur and phosphorus is required due to the effect they have on catalytic converters and increasingly rigorous emissions regulations. ${ }^{19}$ The metal oxides of these elements are believed to come from the additives of lubrication and will block filters and reduce the effectiveness of catalysts in converters. ${ }^{19}$

SAMs are thought to be a new lubrication solution and the relatively simple procedure for SAMs has definitely inspired researchers to develop new lubrication solutions in order to meet new regulations. Lubrication systems for tribological contacts such as engines have had SAMs applied with good effect. The ability of SAMs to form on a variety of surfaces has

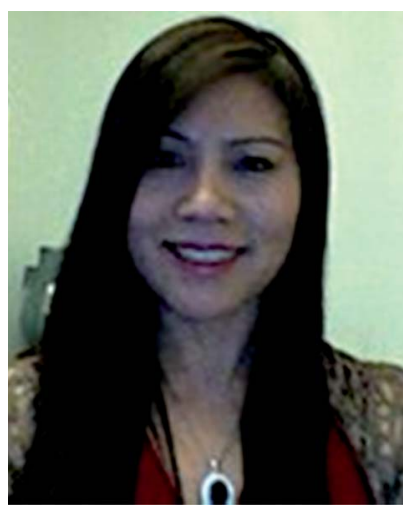

Ling Wang is an Associate Professor in condition monitoring of tribological systems at the national Centre for Advanced Tribology at Southampton (nCATS), Engineering Sciences Academic Unit, Faculty of Engineering and the Environment. She received her BSc and MSc in Chemistry from Nankai University China and her PhD in system engineering from Nottingham Trent University $U K$ before she joined University of Southampton in 2001. She has published over 80 peer reviewed papers in chemistry, tribology, sensing and condition monitoring. attracted tremendous efforts to investigate applications of SAMs in surface engineering and tribological systems, such as artificial joints, orthopaedic implants, corrosion protection and friction reduction. ${ }^{\mathbf{2 0 , 2 1}}$ Lubrication solutions based on SAMs have proved to be successful, especially for nanoelectromechanical systems and microelectromechanical systems (NEMS/MEMS), where normal lubrication methods are not suitable. ${ }^{22-24}$

Since SAM is typically only a few nanometres thick, its mechanical properties such as shear resistance may not be sufficient for frictional contacts. ${ }^{25,26}$ Polymer brushes could be considered to be a new lubrication solution. Polymer brushes can reduce friction to very low levels with increased resistance to shearing in addition to higher resistance to compressive forces. Controlled radical polymerisation techniques such as atom transfer radical polymerisations, have proven to produce high density, thick polymeric films capable of successfully reducing friction. ${ }^{27,28}$ Developments such as activators regenerated by electron transfer and surface attached initiators further strengthen the possibilities of polymer brushes. However these processes may be expansive to be easily adopted as an alternative to existing lubricants.

This paper reviews the development of SAMs and polymer brushes and their potential and challenges as a lubrication solution for tribological applications.

\section{SAMs}

Self-assembled monolayers (SAMs) are molecular assemblies formed spontaneously on surfaces by adsorption that are organised into ordered domains. SAMs were first reported by Zisman in 1946, where a monolayer was formed on a clean metal substrate to change the wettability of the surface. ${ }^{29}$ Unfortunately little recognition of SAMs was gained till 1983, when Nuzzo and Allara discovered that SAMs can be prepared on gold by adsorption of di- $n$-alkyl disulfides from a diluted solution avoiding the use of moisture-sensitive chemicals and crystalline metal surfaces. ${ }^{30}$ Over the past 30 years,

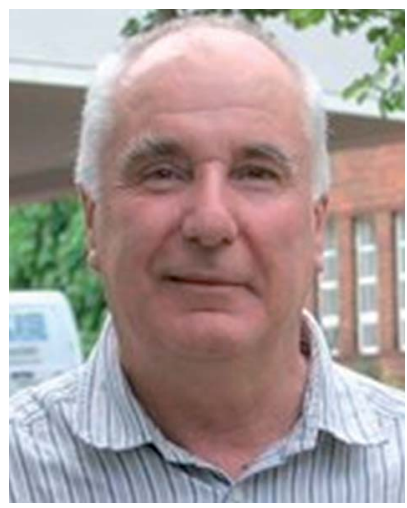

Keith Stokes is a Visiting Professor and long term collaborator with the University of Southampton, latterly with the national Centre for Advanced Tribology. He works at the Defence Science and Technology Laboratory and his main research interests include corrosion issues associated with marine, land and air environments, surface technologies and cathodic protection. He has instigated several high profile projects at nCATS including European Defence Agency projects, Microfluidics, High Strain Body Armour and others. 
a tremendous amount of research has been carried out to develop various SAMs and to investigate their functions and applications.

This section describes the formation mechanisms of SAMs on solid surfaces, the factors that are found to influence the quality of SAMs, the techniques that have been used to characterise SAMs and the challenges and the state-of-the-art development of SAMs as a lubrication solution.

\subsection{SAM formation mechanisms}

SAMs can be formed on surfaces either from a solution or though vapour deposition, while the former is more popularly used. Having selected a particular chemical for a SAM, it is usually dissolved in an appropriate solvent before a clean substrate is immersed or dipped into the solution for the monolayer to 'grow'. The growth rate and the structure of the SAM not only depend on the type of the molecule and the surface chemistry of the substrate, but also the concentration of the molecule and immersion time. ${ }^{17,31-34}$ Details of these factors will be discussed in the following sections.

A number of typical SAM structures are shown in Fig. 1, illustrating some choices of the chemicals (e.g. a variety of head groups, chain/spacer types and functional/end groups) and the types of substrates being investigated. ${ }^{17,31-34}$

All SAMs are formed in a similar way, i.e. usually through various types of adsorption through head group-substrate interactions. The reactions between the molecule and the substrate can however be complicated, depending on the chemistry of the two. For example, the reactions of a trichlorosilane and trimethoxysilane with a hydroxyl group on a surface and their by-products are shown in eqn (1) and (2). It can be seen that the trichlorosilane SAM has a hydrochloric acid by-product while the trimethoxysilane produces a methanol. This has to be carefully considered when choosing SAMs for engineering applications especially when corrosion is a problem. In another example for thiols on gold, a two-step reaction takes place, shown in eqn (3) and (4). ${ }^{21}$ The thiol is firstly physisorbed onto the Au surface followed by S-H cleavage and chemisorption.

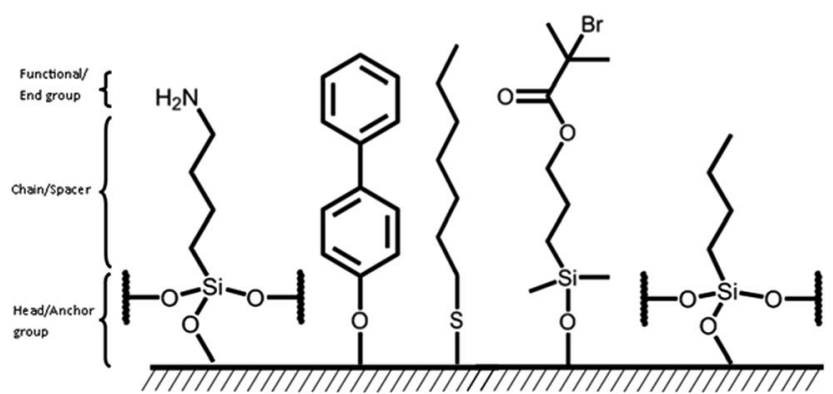

Substrates: $\mathrm{Au}, \mathrm{Si}, \mathrm{SiO} 2, \mathrm{Fe}, \mathrm{Ti}$

Head/Anchor groups: $-\mathrm{SH},-\mathrm{SiCl} 3,-\mathrm{OH},-\mathrm{Si}(\mathrm{CH} 3) 2 \mathrm{Cl},-\mathrm{Si}(\mathrm{OCH} 3) 3$

Chain/Spacer: Alkyl, Aryl, Ethyleneglycol

Functional/End group: $\mathrm{NH} 2, \mathrm{CH} 3, \mathrm{COOH}, \mathrm{NO} 2, \mathrm{OH}$

Fig. 1 A schematic illustration of SAMs formation.

$$
\begin{aligned}
\mathrm{R}-\mathrm{SiCl}_{3}+3(-\mathrm{OH}) & \rightarrow \mathrm{R}-\mathrm{Si}-\mathrm{O}_{3}-+3 \mathrm{HCl} \\
\mathrm{R}-\mathrm{Si}\left(\mathrm{OCH}_{3}\right)_{3}+3(-\mathrm{OH}) & \rightarrow \mathrm{R}-\mathrm{Si}-\mathrm{O}_{3}-+3 \mathrm{CH}_{3} \mathrm{OH} \\
\mathrm{R}-\mathrm{SH}+\mathrm{Au} & \rightarrow \mathrm{R}-\mathrm{SH}_{\mathrm{Phys}} \mathrm{Au} \\
\mathrm{R}-\mathrm{SH}_{\mathrm{Phys}} \mathrm{Au} & \rightarrow \mathrm{R}-\mathrm{S}-\mathrm{Au}+\frac{1}{2} \mathrm{H}_{2}
\end{aligned}
$$

2.1.1 SAM formation kinetics. Apart from surface preparation conditions, solvent and concentration of SAM solutions, the kinetics of SAM formation are also influenced by the types of SAM molecules and substrates. ${ }^{35}$

Growth rate. As concluded by many researchers, the first minute of immersion has been found to be the most important time where SAMs grow at the highest rates. As Aswal et al. showed in their studies, an 85\% coverage of an octadecyltrichlorosilane (OTS) monolayer on silicon was achieved within 50 s of immersion. ${ }^{36}$ In a similar study of OTS on silicon, Balgar et al. also achieved approximately $80 \%$ coverage within 1 minute of immersion. ${ }^{37}$ The reactivity of head groups has been found to affect their adsorption rate. For example, when OTS and octadecyltrimethoxysilane SAMs were formed on silicon nitride under the same conditions, a complete monolayer of OTS was formed after 5 minutes of immersion while the octadecyltrimethoxysilane took 120 minutes due to the more reactive head group of OTS. ${ }^{17}$ This is because the bond dissociation energies required for OTS to replace the groups attached to the silicon atom with $\mathrm{OH}$ groups prior to adsorption are much lower than that for octadecyltrimethoxysilane. ${ }^{38}$

The formation of SAMs for both thiols on gold and silanes on silicon is found to follow a same two-step process (illustrated in Fig. 2, using information from ref. 29 and 39).

(1) Step 1 is a fast linear growth step following the Langmuir adsorption model. The growth in this step is limited by headsubstrate interaction but can achieve up to $85 \%$ of the maximum coverage and near the maximum achievable contact angle. The duration of this step can range from a few seconds to a few minutes depending on the concentration of the precursor molecules.

(2) Step 2 is a slow growing process and can take hours to complete. During this step, the SAM coverage plateaus and reaches to its maximum eventually. The maximum coverage is limited by adsorption from solution such as chain disorder interference.

During the self-organizing process, step 2, the molecules in SAMs rely on weaker and less directional bonds, such as ionic

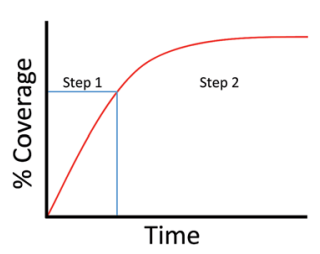

Fig. 2 An illustration showing the two-step formation process of SAMs. 
bonds, hydrogen bonds, and van der Waals interactions, to organize atoms, ions or molecules into ordered structure, with the molecules or ions adjusting their own positions to minimize the thermodynamic energy. That's to say, the kinetics and equilibrium of SAM formation involve a delicate interplay between molecule-solvent interactions, substrate-adsorbate interactions, non-bonded interactions between adsorbates, and intra-molecular interactions such as bond stretches, angle bends, and torsion. Both chemisorption and intra- and interchain non-bonded interactions (e.g., van de Waals, steric, repulsive, and electrostatic forces) contribute to the packing and ordering of molecules in SAMs. The conformation of the individual chains within the assembly, and their packing and ordering with respect to each other depend on the balance between inter-chain forces, the interactions with the surface, and the entropic effects as well.

Growth characteristics. Bierbaum et al. found that OTS monolayers appeared to grow from island like concentrations of SAM. ${ }^{35}$ Similarly, Balgar et al. and Aswal et al. found that OTS as well as other long chain SAMs grew in an island-like model but not short chain SAMs. ${ }^{36,37}$ This is known as island nucleation growth.

In another experiment, Bierbaum et al. found that propyltrichlorosilane reacted with a clean silicon wafer extremely quickly and therefore it was not possible to determine whether island growth had occurred, possibly due to the shorter chain length not obscuring other sites for SAM adsorption. ${ }^{35}$ They also found that propyltrichlorosilane did not achieve expected levels of contact angle for a $\mathrm{CH}_{3}$ terminated SAM and suggested that the disordered monolayer was formed by short chain SAMs, which have small van der Waals forces that were not sufficient to force chains into order. ${ }^{40}$
As shown in Fig. 3, the OTS SAMs were grown initially from nucleation points on the silicon wafer where single molecules attached to the substrate, subsequent growth from these molecules is visible in $20 \mathrm{~s}^{37}$ The growth of a single island was indicative of diffusion limited aggregation (DLA). DLA is a process of aggregation formed by diffusion, where a mobile molecule will contact an already adsorbed molecule and form a cluster. ${ }^{41}$ As the cluster grows an irregular shape can be formed. Branched structure can also be formed due to the low probability of a molecule contacting the middle of the cluster. The irregular shape is known as a fractal shape and is related to DLA. ${ }^{37,42,43}$ However, adsorption in a solution can be considered to be a $3 \mathrm{D}$ adsorption model and the fractal shape may not be as pronounced. The cluster has been found to be the same height as the final monolayer, indicating that the molecules are "standing up" and held in ordered domains by van der Waals forces. ${ }^{37}$ After reaching to a certain island size, new islands will be formed rather than adding to the existing ones. This continues until the monolayer is complete. Fig. 3 shows the formation of an OTS monolayer from agglomerations to fractal shape to near full monolayer. Between 20 and 50 seconds it is clear that islands are growing, it is also notable that there are smaller islands appearing that are represented by small white dots. From 75 seconds the islands are seen to grow until they are indistinguishable from others. In excess of 90 seconds it would be expected that the monolayer would form in full.

Apart from OTS, ${ }^{31,35-37,44-46}$ the island nucleation growth has also been seen in the SAM formation of C12 alkylsilane, ${ }^{47}$ 3-aminopropyltrimethoxysilane, ${ }^{\mathbf{4 8}}$ octadecylphosphonic acid, ${ }^{\mathbf{4 3 , 4 9}}$ octadecyltrimethylammonium bromide, ${ }^{\mathbf{4 3}}$ (3-glycidoxypropyl)trimethoxysilane, ${ }^{50}$ octadecylamine, ${ }^{51}$ and 6-(3-triethoxysilylpropylamino)-1,3,5-triazine-2,4-dithiol monosodium. ${ }^{52}$
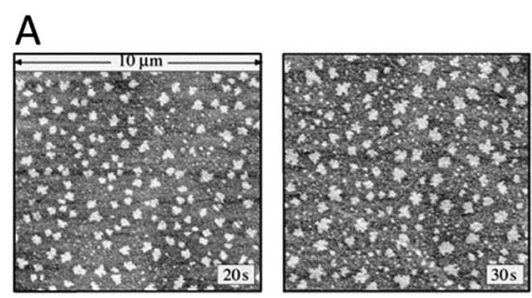

B
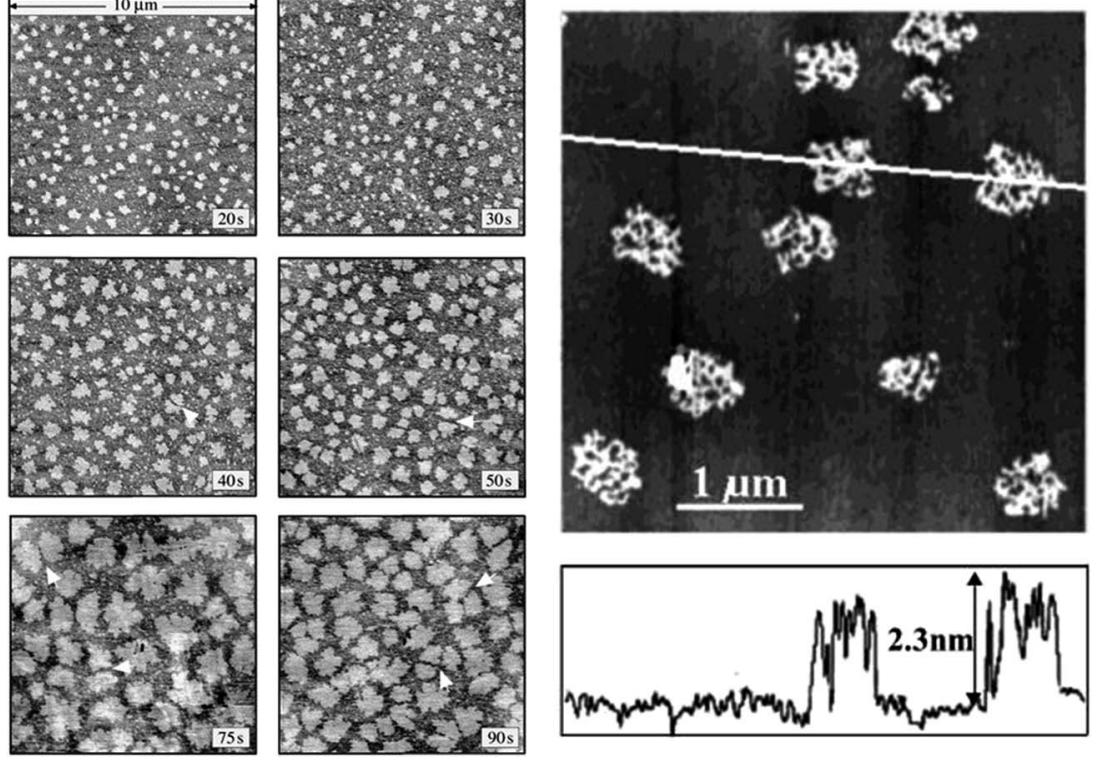

Fig. 3 (A) OTS monolayer growth over time, from partial island growth to full monolayer. $10 \mu \mathrm{m} \times 10 \mu \mathrm{m}$ sample on Si wafer. Reprinted with permission from ref. 37. (B) Is a line profile of the OTS monolayer clusters showing heights which coincide with the theoretical SAM height. Reprinted with permission from ref. 53. 
Although thiols follow the same adsorption steps as silanes described in Fig. 2, they appeared to have different growth characteristics. Thiols are thought to go through a number of steps as illustrated in Fig. $4:^{21,54}$

(A) Thiol molecules are physically adsorbed onto the surface, see eqn (3).

(B) Thiol molecules are covalently attached to the substrate and are in the "lying down" phase, see eqn (4).

(C) As more molecules adsorb onto the surface, thiol molecules become denser and start the "standing up" phase.

(D) The complete ordered monolayer is formed as more molecules from solution adsorb.

\subsection{Factors influencing SAM formation}

The growth rate and structure of SAMs are found to be affected by a large number of factors such as substrate surface preparation and cleaning process, concentration of the chemical, type of solvent, immersion time, humidity, and substrate orientation etc. $^{\mathbf{1 7 3 1 - 3 4}}$ This section reviews influence of key factors on SAM formation.

2.2.1 Surface preparation. As mentioned above, the kinetics and equilibrium of SAM formation heavily rely on the surface chemistry and the type of molecule. Therefore, the surface chemistry and cleanness of the substrate would play a determinant role in the formation process and the quality of the formed SAMs. It has been found that dust and chemical residues on the substrate during surface cleaning can have a detrimental effect on the quality and coverage of SAM. A range of techniques have been adopted in surface preparation prior to creating SAMs.

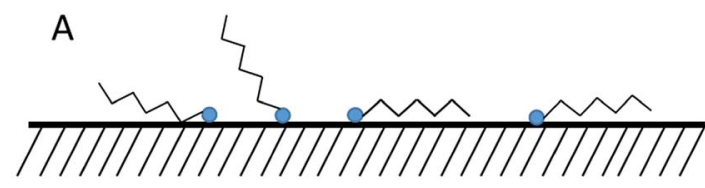

B

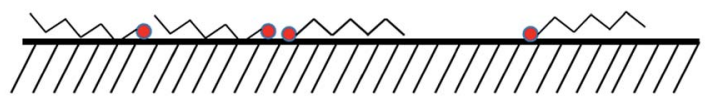

C

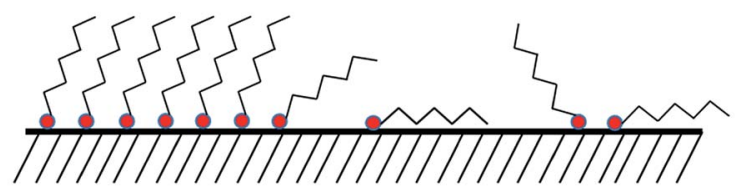

D

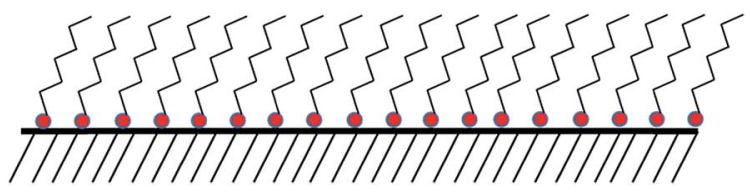

Fig. 4 An illustration of thiol based SAM growth on gold from physisorption, covalent bonding, "standing up" to a complete ordered monolayer.
A standard surface cleaning procedure involves ultrasonic cleaning in different solvents to remove surface containments and blow drying to get rid of solvents residues and dusts, but an additional step of hydroxylation is usually needed for siliconbased or metal oxides substrates. During the ultrasonic cleaning, the commonly used solvents are toluene, isopropanol, chloroform, acetone, deionised water, and ethanol. A normal procedure would involve cleaning the surface in a polar then nonpolar solvent to remove as many residues as possible. After the surface is cleaned, it is rinsed in deionised water then dried in air, argon or nitrogen. ${ }^{17,55-62}$ For systems that require a highly hydroxylated surface to form high quality SAM a surface oxidation treatment is also employed using either piranha or plasma.

Piranha hydroxylation treatment uses a solution of concentrated sulphuric acid (98\%) and hydrogen peroxide $(30 \%)$ at a ratio of $7: 3,4: 1$ or $3: 1 .^{31,32,44,46,52,55,57,58,62-76}$ Samples are typically immersed in the solution at a temperature between 60 and $90{ }^{\circ} \mathrm{C}$ for duration of 30 to 60 minutes, although majority of the studies chose to immerse their samples at $90{ }^{\circ} \mathrm{C}$ for 30 minutes. The piranha treatment has been found to be an effective method to create a good $\mathrm{OH}$-terminated surface where very low water contact angles of less than $10^{\circ}$ can be achieved.

The plasma process is conducted through an oxygen plasma treatment, where suitable hydroxylation can be achieved within a few minutes. Plasma treatment will leave silicon-based substrates hydroxyl terminated in significantly less time than piranha. ${ }^{66,77-79} \mathrm{Wu}$ et al. concluded that 10 minutes plasma treatment on silicon wafers resulted in a water contact angle of less than $5^{\circ}$ indicating a very good $\mathrm{OH}$-termination coverage. ${ }^{\mathbf{8 0}}$ Wiegand et al. also achieved similar contact angles on silica (100) surface using plasma treatment in less than 5 minutes. ${ }^{\mathbf{8 1}}$

As an extra surface preparation, hydrofluoric acid (HF) is sometimes used as an etchant to remove native oxide layer for one of two reasons. For example, hydrofluoric acid can be used to etch silicon dioxide leaving the substrate hydrogen terminated. Some authors used this treatment to enable the attachment of monolayers directly to the silicon atoms. ${ }^{22,82,83}$ However, the monolayers formed are not strictly self-assembled as they require $\mathrm{UV}$ induced coupling or elevated temperatures. ${ }^{\mathbf{8 4 - 8 6}}$ Another reason surface etching using HF is to reform an oxide layer with a consistent depth by controlling the immersion time in piranha. Wang et al. achieved roughness of less than $0.5 \AA$ that allowed them to grow ultra-smooth monolayers. ${ }^{\mathbf{4 6}}$

Although similar results are achievable from the piranha and plasma treatments, the former is more popular mainly due to the implementation of standard laboratory chemicals with a facile method. However, piranha is known to be a dangerous solution which requires careful handling and disposal. Any contact with organics can result in explosions and improper storage of waste in sealed containers have both resulted in injuries. Conversely, plasma treatment instruments add additional costs to processing substrates and for ultra-clean wafers it is known that clean room conditions are favourable. The influences of these techniques will be further discussed later in this review. 
2.2.2 Solvent selection. The solvent, in which SAM precursor molecules are dissolved, is also paramount to the successful adsorption of SAMs, as solvent properties, including polarity, solubility, molecular diameter and viscosity, can affect solvent-substrate interactions and solvent-adsorbate interaction during SAM formation. Lee et al. observed with scanning tunnel microscopy (STM) that octylthiocyanate (OTC) SAMs formed on $\mathrm{Au}$ (111) in ethanol had a structure of mixed phases composed of ordered domains and disordered phases, but those formed in dimethylformamide (DMF) and toluene exhibited long-range ordered domains. ${ }^{87}$ Manifar et al. explored the effect of solvent on the formation of OTS SAMs by comparing the contact angle of water on the OTS SAMs using hexane, toluene, ether, dichloromethane (DCM) and tetrahydrofuran (THF) as deposition solvents after immersion in 10 $\mathrm{mM}$ solutions for 6 hours. Toluene outperformed the other solvents by producing a surface with a contact angle of more than $10^{\circ}$ higher. By comparing dipole moments and partial charge distribution of OTS and the used five solvents, the authors postulated that solvent molecules with large dipole moments and being highly charged can help the formation of more uniform SAMs with the capability of hydrogen-bonding and highly localized partial charge which facilitate the attachment of OTS onto the surface. ${ }^{32}$ Rozlosnik et al. compared toluene with heptane and dodecane and found that multilayers were formed using dodecane. ${ }^{53}$ However, it is thought that the multilayers were physisorbed and could be easily removed by a glove dipped in hexane. Heptane also produced a full coverage of OTS SAM similar to other solvents, however toluene was still regarded as the best. ${ }^{53}$ The reason for this is due to different solubility of water in these solvents, which can affect adsorption process of OTS on the hydrophilic silicon oxide surface.

In addition to solvent choice, water content is of a particular concern, especially for silanes, since the presence of water is required for initial hydrolysis of trichlorosilane group. However, too much water present in the solvent leads to polymerization of OTS in bulk solution, which competes with the surface reaction of single alkylsilane molecules for the monolayer formation. ${ }^{\mathbf{8}}$ McGovern et al. stated that the optimum water content in a solvent is $0.15 \mathrm{mg} / 100 \mathrm{~mL} .^{89}$ The authors also found that less moisture can facilitate the formation of well-defined monolayer on the silicon substrate but slower adsorption kinetics, sometimes resulting in an incomplete monolayer. In anhydrous conditions, even in an argon-filled glove box, water is still present as a layer adsorbed on the silicon oxide surface, which can assist hydrolysis of chlorosilanes but confine the reaction to the oxide layer. ${ }^{\mathbf{3 9}, 90}$

2.2.3 SAM precursor concentration. From points of view of kinetics and equilibrium of the SAM formation, the concentration of the precursors in the deposition solution would not only influence the SAM growth rate but also the structure of the SAMs. A comprise between the deposition time and the SAM quality is usually needed in order to achieve the desired surface properties in a reasonable time. For example, thiol based SAMs can be formed on gold in concentrations of $1 \mu \mathrm{M}$, however this can take up to a week to form a densely packed monolayer. ${ }^{91}$ In practice, a considerably higher concentration may be used to reduce the time required. It has been found that incomplete but notable monolayers can form under a minute at a concentration of $1 \mathrm{mM}$, see growth rate for more details. ${ }^{29}$ Kulkarni et al. investigated the influence of SAM precursor solution concentration on the rate of OTS adsorption on silicon. ${ }^{31}$ They tested a concentration range of OTS in toluene from $0.05 \mathrm{mM}$ to $1 \mathrm{mM}$ and found that the higher the concentration the faster the monolayer was formed. However, a full coverage was achieved by all concentrations after extended immersion durations. For example, a full SAM film was formed after $16 \mathrm{~h}$ of immersion in the $0.05 \mathrm{mM}$ solution. ${ }^{31}$ Wang et al. studied formation of OTS SAMs on silicon nitride and found that $2.5 \mathrm{mM}$ was an optimum concentration for a good coverage over the range of $0.1-50 \mathrm{mM}$ tested. ${ }^{17}$ However, high SAM solution concentrations may lead to multilayer formation instead of the desired SAMs. Rozlosnik et al. found that high concentrations of OTS in poor solvent led to multilayers that were irregular or poorly distinguished..$^{53}$ It is generally considered that concentration and immersion time are inversely related but high concentrations and short immersion times promote monolayer growth. ${ }^{91}$

2.2.4 Summary. There are a large number of factors that influence SAM formation. Apart from the ones discussed above, it is also important to understand the substrate because it dictates the selection of head groups. For example, $-\mathrm{SH}$ is suitable for $\mathrm{Au} / \mathrm{Ag} / \mathrm{Pt}$ etc. and silanes are more appropriate for oxide surfaces. It becomes more complicated if by-products from the reactions are also taken into account. For example choosing trimethoxysilanes over trichlorosilanes can avoid the production of corrosive hydrochloric acid. Silanes generally require extensively oxidized surfaces for stability hence plasma or piranha treatment can significantly improve the SAM quality. SAM growth is affected by both the solution concentration and immersion time. In general, a concentration between 1-5 $\mathrm{mM}$ is sufficient to form a good quality monolayer without wastage. Apart from the factors reviewed here, other factors such as humidity, age of SAM solution and substrate orientation, will also play a part in the fabrication of a perfect SAM.

\subsection{Characterisation techniques}

Being an extremely thin layer of a few nanometers on a surface, it is difficult to characterise and quantify SAMs. Over the years, a range of techniques have been used to confirm the formation of SAMs and characterise SAM properties, summarised in Table 1. Among them contact angle measurements, atomic force microscopy (AFM), X-ray photoelectron spectroscopy (XPS) and scanning tunnelling microscopy (STM) are most commonly used.

2.3.1 Contact angle measurements. Contact angles are used to check the 'wettability' of surfaces, which is known to influence the friction and wear in tribological contacts of surfaces, and the effectiveness of SAMs in changing the surface's functionality. ${ }^{67,92-96}$ In addition, contact angle can give a representation of the coverage and quality of a SAM. Normally a deionised water droplet of a few microliters is pipetted onto the substrate of interest and the contact angle is measured 
Table 1 A summary of the properties of SAM that can be collected through a selection of techniques ${ }^{a}$

\begin{tabular}{|c|c|c|c|c|c|c|c|c|c|c|c|c|c|c|}
\hline Properties of SAM & & XPS & $\mathrm{AFM} / \mathrm{STM}$ & $\mathrm{XRD}$ & Ellipsometry & IR & Raman & SFG & UPS & LEED & SIMS & SEM & RBS & ISS \\
\hline \multirow[t]{2}{*}{ Coverage } & Overall coverage/thickness & $x$ & $x$ & $x$ & $x$ & $x$ & & & & & & & & \\
\hline & Pinholes & $x$ & $x$ & & & & & & & $x$ & $x$ & $x$ & $x$ & $x$ \\
\hline & Surface & $x$ & & & & & & & & & & & & $x$ \\
\hline & Conc. profile & $x$ & & & & & & & & & & & $x$ & $x$ \\
\hline \multirow[t]{4}{*}{ Functional groups } & Bonding & $x$ & & & & $x$ & $x$ & $x$ & $x$ & & $x$ & & & \\
\hline & Conformation & & & & & $x$ & $x$ & $x$ & $x$ & & & & & \\
\hline & Average composition & $x$ & & & & $x$ & $x$ & $x$ & $x$ & & $x$ & & & \\
\hline & Depth profile & $x$ & & & $x$ & & & $x$ & $x$ & & $x$ & & $x$ & $x$ \\
\hline \multirow[t]{3}{*}{ Ordering } & Pinholes & $x$ & & & & & & & & & $x$ & $x$ & $x$ & $x$ \\
\hline & Long-range dislocation & & $x$ & & & $x$ & & & $x$ & & & $x$ & & $x$ \\
\hline & Orientation & & $x$ & & $x$ & $x$ & & & $x$ & & & $x$ & & \\
\hline
\end{tabular}

${ }^{a}$ Note: SFG, sum frequency generation spectroscopy; UPS, ultraviolet photoelectron spectroscopy; LEED, low energy electron diffraction; SIMS, secondary ion mass spectroscopy; RBS, Rutherford backscattering spectroscopy; ISS, ion scattering spectroscopy.

based on the shape of the water droplet formed on the surface as illustrated in Fig. 5, where angle $\theta$ is defined as the contact angle. Hydrophilic surfaces typically are those with contact angles in the range of $0 \leq \theta \leq 90^{\circ}$ whilst hydrophobic ones are $\theta>90^{\circ} .{ }^{97}$ When $\theta$ is over $150^{\circ}$, a surface is considered superhydrophobic. ${ }^{73,97}$

Contact angle measurements have been widely used in SAM characterisation due to its easy access, simplicity and low cost. Comparing the angles before and after surface modification provides an indication of whether and how well a modification has taken place. Some authors have made their own instruments due to the simplicity of the technique as well as carry out unique experimentation. ${ }^{\mathbf{9 8 9 9}}$ Using a homemade instrument, Bormashenko et al. compressed the droplet with a precise moveable stage to study the Cassie-Wenzel transition. Contact angle calculations have also been utilised in surface energy calculations. ${ }^{93}$

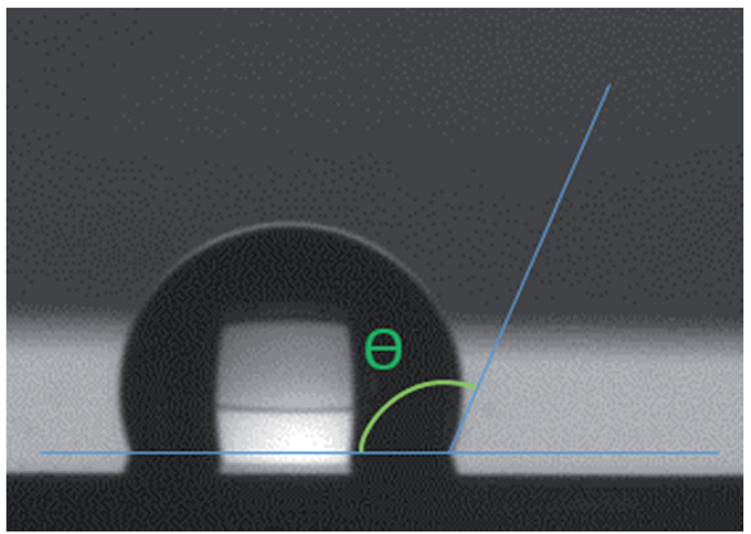

Fig. 5 A sessile drop image detailing how contact angle measurements are made.
For ultra-hydrophilic surfaces $\left(<5^{\circ}\right)$, water droplets may deform on the surface and it is difficult to obtain the contact angles. ${ }^{\mathbf{8 1 , 1 0 0 , 1 0 1}}$ Therefore, other liquids may be selected, such as diiodomethane, 1-bromonaphthalene, formamide, glycerol, or ethylene glycol, ${ }^{\mathbf{9 3 , 1 0 2}}$ to overcome this issue. However, different liquids will result in different contact angles; this is due to the difference in solid-liquid and liquid-liquid interfaces. Strong solid-liquid forces will result in the liquid spreading across a surface and therefore a low contact angle. Contrary, strong liquid-liquid interactions will cause liquids to stick together and therefore reduce contact angle. The surface tension at the solid-liquid interface is due to different intermolecular forces such as hydrogen bonding, polar interactions and acid/base interactions, for this reason changing probe liquid can alter the shape of the sessile drop. Janssen et al. studied 21 different probe liquids on 11 different SAMs in addition to oxidised silicon wafers, where the different interactions and subsequent contact angles were observed. ${ }^{103}$ For example, the contact angle for water and dichloromethane on a silicon wafer was found to be $<10^{\circ}$ and $14.4^{\circ}$ respectively. It is worth noting that the authors also could not consistently fit contact angles below $10^{\circ}$.

2.3.2 AFM. AFM is an instrument that has been used to image SAMs on surfaces, providing topographic information such as surface profile and roughness. In addition, AFM has been used to study the growth of SAM by taking AFM scanning images at different stages, see Fig. 3. 3D AFM images can provide details on the surface including nano-scale defects. ${ }^{\mathbf{1 0 4}}$

For nano-tribological studies of SAMs contact mode AFM is routinely used. Contact AFM investigates the friction between the AFM tip and the SAM surface. It measures single asperity contacts with an ultra-sharp cantilever without being influenced by the effect of surface roughness. ${ }^{23,67,105}$ AFM is able to view both hard and soft surfaces in liquid as well as air, hence images of polymer brushes have been taken without 
collapsing: ${ }^{62,106}$ Fig. 6A shows a 3D AFM image where dodecane residues are clearly seen within a fully covered OTS SAM on a silicon substrate. Although created intentionally Checco et al. show that many solvents can leave residues on surfaces as shown in Fig. $6 .^{107}$ Therefore, for high quality images to be taken these residues must be considered. Fig. $6 \mathrm{~B}$ is an example of an OTS SAM formed on a silicon wafer with a uniform film approximately the known height of OTS. ${ }^{31}$ However, as this substrate was only immersed for 60 seconds it is unlikely that this is the smoothest SAM possible, see SAM formation kinetics. AFM has also been used to determine the thickness of SAM films by line profiles. ${ }^{31,108,109}$ Fig. $6 \mathrm{C}$ and D show an example of line profiling. Fig. 6C is multiply alkylated cyclopentane (MAC) deposited on top of a decyltrichlorosilane monolayer, the authors then concluded that the MAC layer was not uniform. MAC is thought to be a good lubricant due to a selection of desirable properties such as viscosity, volatility, pour point and thermal stability. ${ }^{\mathbf{1 1 0}}$ The droplets measured by AFM sectioning can be seen in $\mathrm{D}$, and the ellipsometry height measurements were found to coincided with AFM vertical difference measurements. ${ }^{108}$

2.3.3 STM. Scanning tunnelling microscopy (STM), similar to AFM, has been used to image surface topography down to the atomic scale. ${ }^{111}$ STM images are created by bringing a small metal tip close to the surface of interest, applying a voltage bias and scanning across the surface. ${ }^{\mathbf{1 1 2 , 1 1 3}}$ The small gap between sample and probe, typically in the order of angstroms, is maintained by the tunnelling effect across the vacuum. ${ }^{114}$ The resultant image is essentially a map of topography. STM has been used to image thiols on gold showing the surface coverage of the SAM as shown in Fig. 7. ${ }^{\mathbf{2 9 , 1 1 5 , 1 1 6}}$ STM is however, limited to characterise molecules with relatively short carbon chains since substantial tunnelling currents are difficult to obtain for carbon chains longer than $12 .{ }^{33}$

2.3.4 XPS. X-ray photoelectron spectroscopy (XPS) is primarily used to characterise the nature of the surface
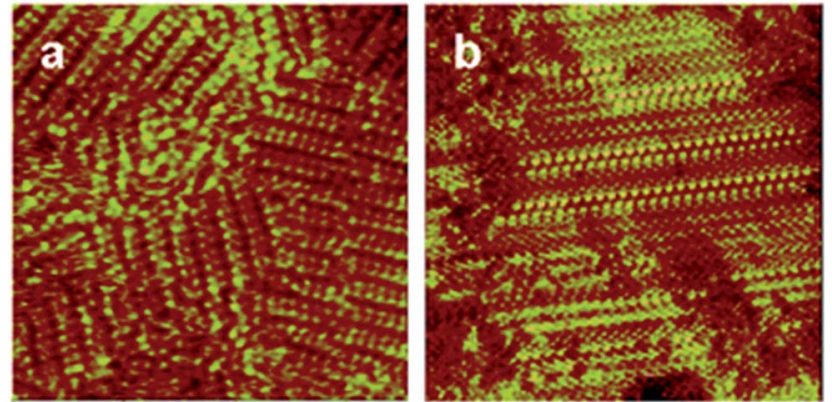

Fig. 7 An STM image of two different phases of hexanethiol on gold. (a) Is a rectangular lattice structure and (b) is striped. Reprinted with permission from ref. 21.
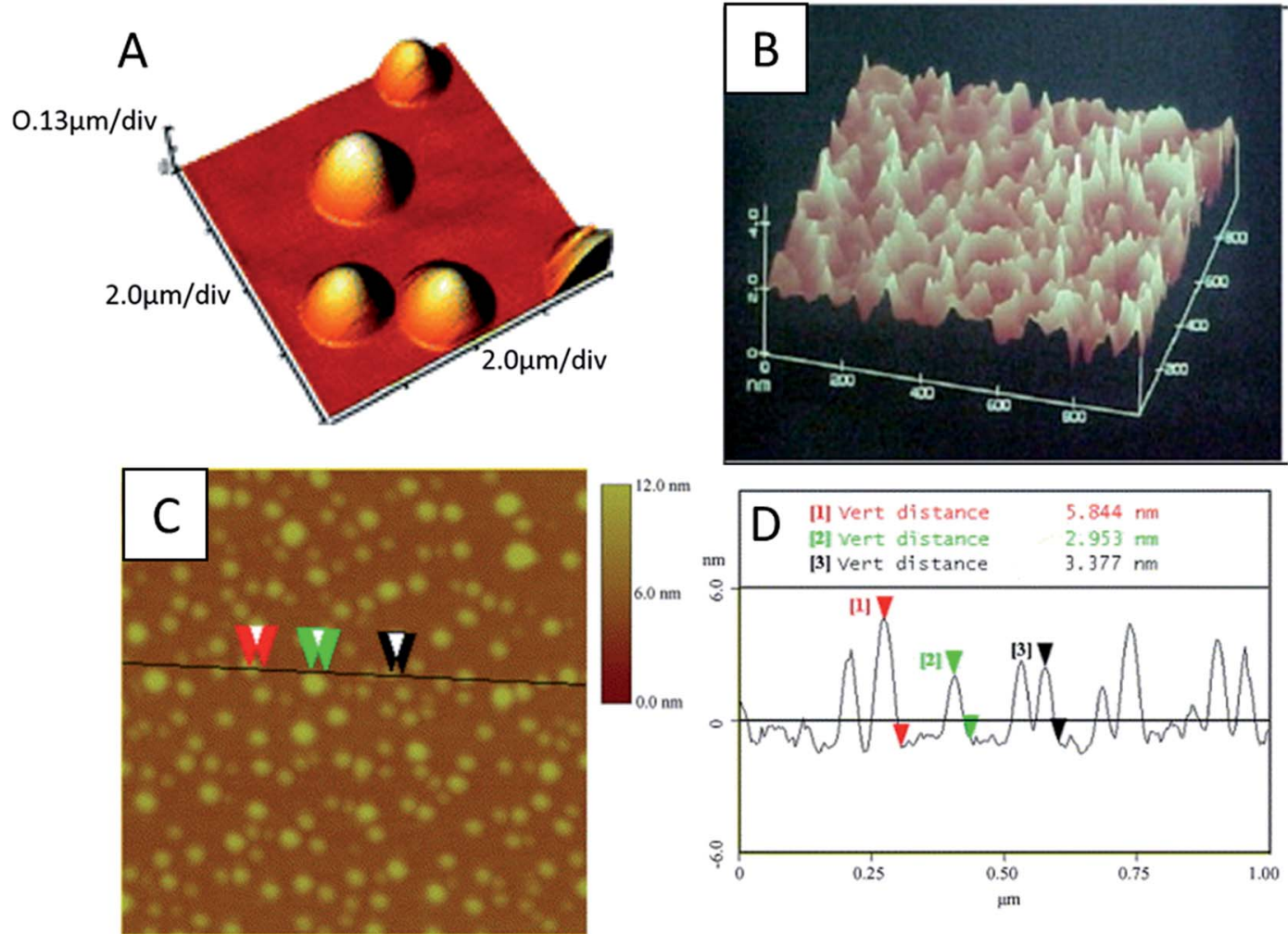

Fig. 6 (A) Is an AFM image showing dodecane residues on an OTS monolayer. Reprinted with permission from ref. 107 Copyright 2006 American Chemical Society. (B) Is a fully formed OTS monolayer on a silicon wafer. Reprinted with permission from ref. 31. (C) Is a 2D image of multiply alkylated cyclopentane on top of a decyltrichlorosilane monolayer. Reprinted with permission from ref. 108. (D) Is the corresponding section analysis with the line profile and markers indicated on (C). 
bonds. ${ }^{21,117}$ XPS is a frequently used technique due to its ability to identify and quantify elemental composition as well as chemical states, see Table $1 .^{118}$ By varying the take-off angle, i.e. the angle that the analyser is in relation to the sample surface, the chemistry of the top most layers of a solid structure can be analysed. ${ }^{119,120}$ These properties make XPS a very useful instrument in the characterisation of SAMs. Fig. 8 shows an example of an XPS spectrum of OTS SAM compared with that of a clean wafer. The lack of a $\mathrm{Cl}$ peak in the monolayer spectra showed the complete hydrolysis of OTS during adsorption. ${ }^{39}$ The large $\mathrm{O}_{1 \mathrm{~s}}$ peak in the wafer spectrum indicated a good level of oxidation on the surface and the significantly larger $\mathrm{C}_{1 \mathrm{~s}}$ peak observed in OTS SAM confirmed the OTS SAM formation.

2.3.5 Ellipsometry. Ellipsometry has been used to identify the thickness of a thin film, for example in measuring the thickness of an oxide layer. It is an optical technique that relies on the variance of polarised light created by the reflection and refraction of a known light source. This technique has shown to be effective to characterize film thickness for single layer or complex multilayer stacks ranging from tenths of a nanometer to several micrometer. Hence, in addition to SAMs, it is also a useful technique in measuring polymer brushes, see details later. ${ }^{121,122,144}$ Since ellipsometry needs a model to fit the raw data and normally does not take into account of surface roughness, small errors may be included in the thickness measurements. Ellipsometry is also used for investigating oxide thicknesses on silicon substrates. ${ }^{\mathbf{1 2 3 , 1 2 4}}$

2.3.6 Summary. A wide range of techniques are available for characterisation of SAMs however each technique has its advantages and limitations. It is important to consider using more than one techniques in SAM characterisation. Contact angle has been the most widely used method due to the low cost associated with the technique but may become impractical for ultra-superhydrophilic surfaces. AFM images have mostly been used to understand the growth of monolayers; it is also routinely used for detailed analysis of surface structure and tribological testing. STM is rarely used due to its limit in SAM chain length. Although XPS is an expensive technique, the wide range of data that can be collected reduces the need for multiple different techniques. Ellipsometry is more useful for polymers

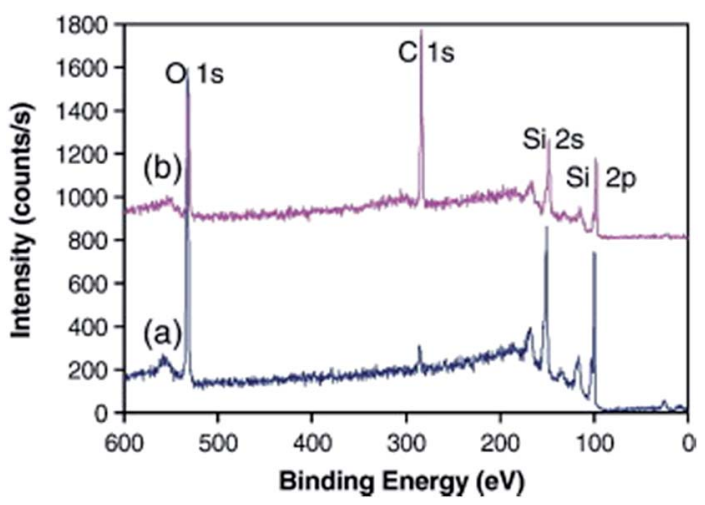

Fig. 8 XPS spectra of (a) a clean silicon wafer and (b) OTS SAM. Reprinted with permission from ref. 39. and can analyse partially formed films height which can reinforce data collected by AFM.

\subsection{Achievements and applications of SAMs}

SAM has shown growth in a number of applications especially in sensors such as $\mathrm{pH}$ sensors, organic and inorganic species detectors ${ }^{125,126}$ and biosensors. ${ }^{127-129}$ As mentioned above, SAMs have also been used as a lubrication solution in MEMS/NMEMs. Many SAM studies have been focused on their formation on silicon wafers, due to their well-defined surface morphology and extremely low surface roughness $\left(R_{\mathrm{a}}<0.001 \mu \mathrm{m}\right)$, which maximises the functions of SAMs and helps SAM characterisation. This is a particular interest of this review due to the authors' interests in silicon nitride surfaces.

The type of SAM molecule limits the contact angle that is achievable. For example, with $\mathrm{CH}_{3}$-terminated SAMs on silicon, the reported water contact angles do not exceed $110-112^{\circ}$ for hydrocarbon tails with more than 10 carbons. This is irrespective of the amount of chlorine atoms attached to the silicon head group. ${ }^{36,130}$ However, greater contact angles can be achieved by introducing fluorine groups and/or structuring surfaces. A facile method of increasing contact angles has been demonstrated by using (tridecafluoro-1,1,2,2-tetrahydrooctyl)-1trichlorosilane and $1 H, 1 H, 2 H, 2 H$-perfluorooctyltriethoxysilane to form SAMs on silicon and nanofibrils with contact angles achieved at $120^{\circ}$ and $130^{\circ}$ respectively. ${ }^{131,132}$ This is because the $\mathrm{CF}_{3}$ groups in SAMs can reduce surface free energy, thereby increasing surface hydrophobicity. Song et al. demonstrated another approach to increase contact angles to the hydrophobic range by coupling SAMs with micro-roughened surfaces. ${ }^{\mathbf{1 3 3}}$

Apart from single molecule type SAM, mixed SAMs have been developed to incorporate two types of molecules in one SAM where one type of species would preclude the other by steric hindrance. ${ }^{91}$ Mixed monolayers have great potential in many different applications including microelectrodes, ${ }^{\mathbf{1 3 4}}$ separation of biomolecules, ${ }^{135}$ environmental monitoring, ${ }^{136}$ biosensors ${ }^{137}$ and tribology. ${ }^{63}$ Feng et al. developed a mixed SAM containing both OTS and octyltriethoxysilane, see a schematic of the mixed SAM in Fig. 9D. ${ }^{65}$ Two methods were used to produce the mixed SAM: co-adsorption and stepwise as illustrated in Fig. 9A-C. Coadsorption is considered the easier of the two methods, where a clean substrate was simply immersed in a solution containing two precursor molecules, Fig. 9A. Due to the different affinities of the molecule headgroups, typically the 'trial and error' approach is required to tailor the concentration and ratio between the two types of molecules to achieve the desired mixed SAM. ${ }^{65}$ It was found not possible to form a mixed SAM using OTS and octyltriethoxysilane but possible for OTS and dodecyltrichlorosilane. ${ }^{138}$ This was due to the steric hindrance of the three ethoxy groups in addition to the quick binding of OTS to the substrate rendering octyltriethoxysilane unable to form structures. ${ }^{65}$

The stepwise method is illustrated in Fig. 9B and C, this method was developed to overcome the problems of mixed coadsorption. Firstly, a partial monolayer was produced by immersing a substrate in a solution of the first SAM forming 


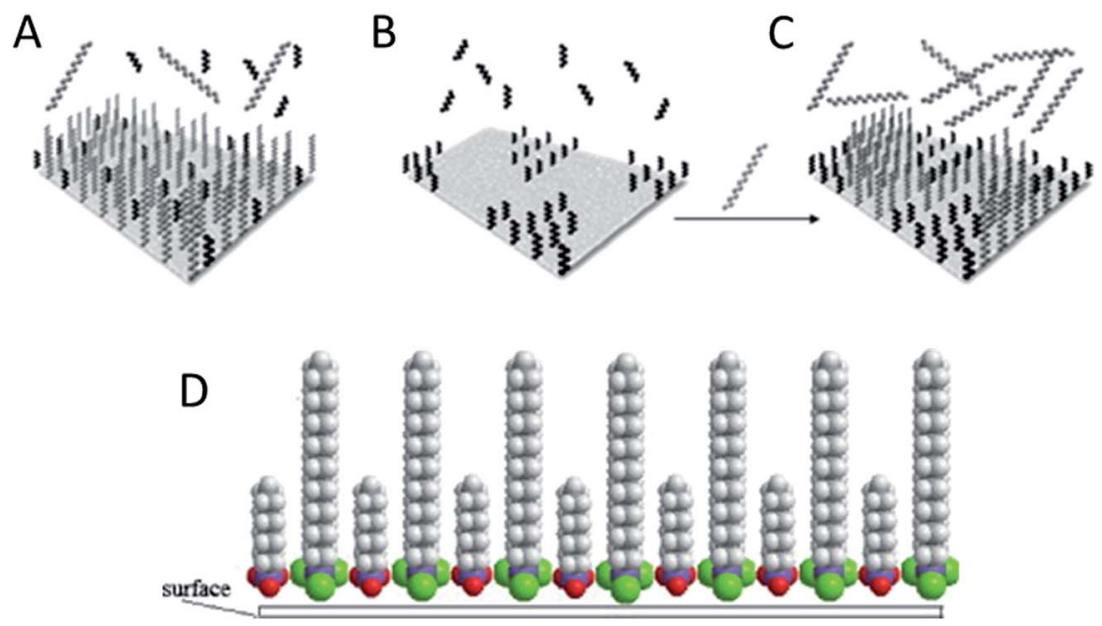

Fig. 9 Showing an idealistic structure for the mixed SAM of OTS and octyltriethoxysilane. Reprinted with permission from ref. 65.

molecules for a known length of time. Then the substrate was immersed in a secondary solution of a different molecule. ${ }^{48}$ Gaps in the first monolayer were filled in by the second SAMforming molecule. ${ }^{\mathbf{4 8 , 6 5}}$ It was recommended that the SAM with a larger head group should be formed first as the smaller steric hindrance of the secondary SAM will allow it to fit in the gaps left by the larger SAM. ${ }^{65}$ Gradient mixed SAM can be created by pumping toluene at a constant rate into a solution of OTS with a partially immersed substrate. ${ }^{63}$ With the addition of more solvent the solution is more dilute meaning longer adsorption times for a full monolayer. ${ }^{31,63}$ This is facilitated by a partially immersed substrate where the addition of more solvent increases solvent level and therefore immerses more substrate. After removal of the partially formed monolayer from one solution, simple immersion in another solution containing (1trichlorosilyl undecyl)trichloroacetate in toluene creates the full monolayer. ${ }^{63}$ However, it has been found that in some cases phase separated islands of monolayer have been formed rather than well mixed monolayers. As previously discussed, the formation of mixed SAMs can be inhibited by molecules selected and therefore multistep adsorption may be required. This could limit applications.

Bilayers and multi-layered SAMs are increasingly seen in research with self-assembling multilayers incorporating other layers such as gold nanoparticles. ${ }^{139}$ Extensive research of bilayers has included self-assembling supported lipid layers, e.g. phospholipid bilayers are found to be useful in studying interactions with cell membranes. ${ }^{\mathbf{1 4 0 , 1 4 1}}$ Supported bilayers of lipids are commonly produced by spreading lipid vesicles on hydrophilic solid supports. ${ }^{\mathbf{1 4 2}}$ To create tethered bilayers authors have formed SAM of thiolipids on gold followed by the lipid bilayer below the critical micellar concentration, i.e. below the point at which micelles forms in solution. ${ }^{\mathbf{1 4 3}}$ Bilayers of thiols and silanes can be formed depending on end group, thiols such as 11-mercapto-1-undecanol will allow silane SAMs to form a bilayer on top although. ${ }^{\mathbf{1 4 4}}$ It is worth noting that $-\mathrm{OH}$ functional groups on the terminal end of SAMs are not as efficient as substrate groups at creating additional monolayers. ${ }^{144}$ OTS has shown the ability to form multilayers in poor solvent on both steel and silicon, however, it is reported that the layers were found to be extremely rough indicating a consistent multilayer has not been formed..$^{\mathbf{5 3 1 4 5}}$ In addition, authors that have used MAC, ${ }^{\mathbf{7 0 , 1 0 8 , 1 1 0}}$ apply the mobile lubricant through spin coating possibly limiting applications in some areas.

\subsection{SAMs for tribological applications}

2.5.1 Overview. SAMs have been implemented into NEMS and MEMs devices with research in OTS lubricated micromotors since the 1990s. ${ }^{146}$ Although SAMs are only in the order of a few nanometres they have shown to act as a boundary lubrication system on both micro and nano scales. ${ }^{105}$ SAMs have also shown to significantly reduce stiction between two substrates which has led to applications in storage devices. ${ }^{\mathbf{6 8 , 1 4 7}}$ As discussed above, a variety of SAM head groups are available for different substrates. For example OTS SAM can be formed on alumina surfaces of components in MEMS/NEMS for lubrication. ${ }^{148}$ SAMs have also been considered for lubrication of aluminium and magnesium engine components via matching metal oxide to head group selection. ${ }^{94}$ Fatty acids have been additives in lubricants for steel components for many years, where carboxylic acids are known to form monolayers on steel surfaces to reduce friction. ${ }^{6}$ Silanes are an attractive alternative to some of the existing friction modifiers that contain sulphur and phosphorus, which is one of the reasons that research in SAMs has grown significantly over the years. ${ }^{17}$

2.5.2 Tribotesters and tribometers for SAM evaluation. A variety of tribotesters can be used to measure the tribological qualities of two surfaces in contact, including wear and friction, such as pin-on-disc and ball-on-disc tribometers. ${ }^{\mathbf{1 4 9}}$ It is, however, very important that tribological testing replicates relevant service conditions. ${ }^{\mathbf{1 5 0}}$ Pin-on-disk has been used in SAM evaluation for replicating linear velocities in one direction for an unlimited amount of time also known as kinetic friction, see Fig. 10B. Depending on the application, different contact geometries can be deployed such as balls or pins. However, some uncertainly during pin-on-disk testing for SAMs has been 
A

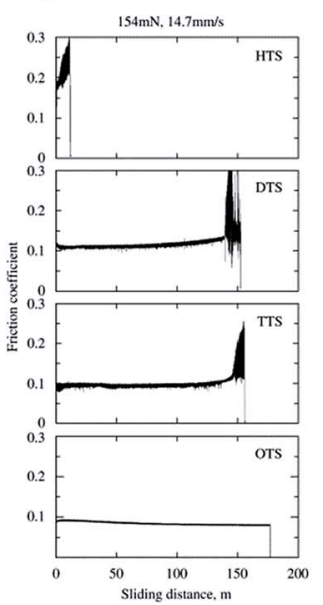

B

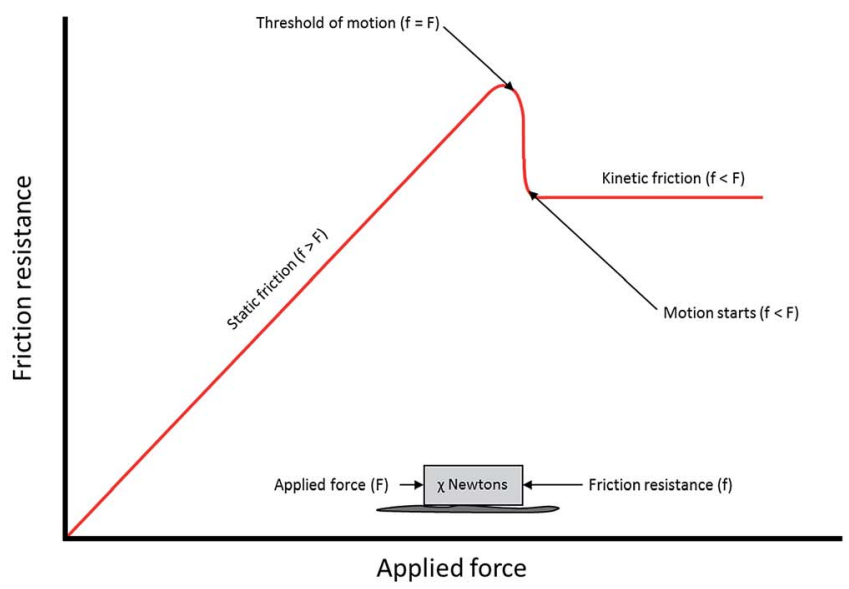

Fig. 10 (A) An illustration of four friction traces detailing the COF of different SAMs obtained from pin on disk experimentation. (B) Is a simplified standard model of friction $(A)$ is reprinted with permission from ref. 56.

reported. Novak et al. found that misalignment of pin geometries increase error along with other pin on disk measurement uncertainties, others including Burris et al. and Schmitz et al. have also studied uncertainty using reciprocating tribometers. ${ }^{151-153}$ Fig. 10 shows an example of friction traces for 4 different SAMs, where a sudden increase in the COF is seen when the lubricating properties of these SAMs do not exist anymore. ${ }^{56}$ Linear reciprocating tribometers are used to replicate many engineering contacts such as piston ring on liner in automotive engines. ${ }^{154}$ These tribometers generally have a pin of a known geometry sliding across a plate under load over a known distance, reciprocation allows friction to be recorded in both the forward and backward motions. ${ }^{\mathbf{1 5 5}}$ Measurements of static COF are also possible to be measured, see Fig. 10B. ${ }^{156}$ This can be measured where the contact is momentarily stationary. Microtribometers have also been used in SAM tribological testing especially for applications where small forces are used, such as MEMS devices. ${ }^{157,158}$ AFM nanotribological studies are frequently being used to investigate contacts at atomic level. ${ }^{67,159-161} \mathrm{AFM}$ is useful to gain understanding of asperity contact in sliding contacts. ${ }^{159}$

\subsection{SAMs on silicon for COF reduction}

\subsubsection{Single component SAMs on silicon}

OTS SAM. OTS SAMs have been extensively investigated for tribological performance improvement of silicon wafers in a wide range of load and speed. ${ }^{63,71,75,162-164}$ Using a steel ball and forming OTS SAMs on silicon wafer disks, Cha et al. recorded COF values in the range of 0.1 to 0.2 over a dry sliding distance of $75 \mathrm{~m}$ under a load of $50 \mathrm{mN}$ for immersions in excess of one hour. No damage was found on the wafer discs for longer immersion compared with the wear tracks under $10 \mathrm{~s}$ and $1 \mathrm{~h}$ immersion. The authors found that immersion times of longer than 5 hours had little effect on the COF recorded, however relative humidity proved to influence COF. A higher humidity resulted in a higher $\mathrm{COF}$. Booth et al. found that the tribological properties of single-component OTS monolayer were dependent upon the surface coverage and surface energy of the gradient monolayer. ${ }^{63}$ They also demonstrated that the COF can be further reduced to under 0.1 on the OTS SAM fully covered silicon wafer under $98 \mathrm{mN}$ loading, similar to the studies by DePalma et al. ${ }^{163}$ Satyanarayana et al. also demonstrated that with a stainless steel ball, COF of OTS SAM modified silicon disc can be reduced to 0.06 under $0.5 \mathrm{~N}$ compared to unmodified silicon (0.2-0.3). ${ }^{75} \mathrm{Ma}$ et al. also performed similar tests under a load of $0.5 \mathrm{~N}$, but found the COF stabilised at 0.13 on the OTS SAM modified silicon. ${ }^{70}$ However, the authors found the OTS SAMs instantly breakdown and failed under a higher load of 1 N. Garcia-Parajo et al. studied OTS SAMs on silicon with force distance curves and compressive forces, and proposed that rearrangement of OTS under compression and the subsequent reformation after load was removed contributes to the lubricity of OTS SAMs. ${ }^{165}$ Flater et al. demonstrated that OTS SAMs can reduce adhesion of silicon wafers through pull off measurements using AFM, and also noticed that friction is reduced further when OTS SAMs are created on both the cantilever and substrate. ${ }^{\mathbf{1 6 4}}$

Influence of chain length and head group. A number of studies have been conducted to investigate the influence of chain length and head group type on their tribological properties. Singh et al. studied trichlorosilanes with 6,10 and 18 carbons chain lengths using a reciprocating ball on plate configuration with a silicon nitride ball under a load of $4 \mathrm{mN} .{ }^{166}$ At a $1 \mathrm{~mm} \mathrm{~s}^{-1}$ sliding speed over a $3 \mathrm{~mm}$ track OTS SAMs produced a COF of less than 0.1, whereas DTS and HTS produced COF values of approximately 0.15 and 0.25 respectively. The performance of the three SAMs was also compared using a contact AFM under $40 \mathrm{nN}$ and $2 \mu \mathrm{m} \mathrm{s}^{-1}$ scan rate. Their performance in terms of friction was ranked as OTS > decyltrichlorosilane (DTS) > hexyltrichlorosilane (HTS), where OTS had the lowest COF. Masuko et al. performed similar investigations using 6, 10, 14 and 18 carbon chain lengths and found a COF of under 0.1 for OTS SAMs using ball on disk under $52.1 \mathrm{mN}$ load at a sliding speed 
of $3.53 \mathrm{~mm} \mathrm{~s}^{-1}$. Longer chain SAMs have been shown to have significant freedom of swing allowing rearrangement to the direction of shear stress, thus lowering COF. ${ }^{56,70,166}$

By replacing one or two $\mathrm{Cl}$ atoms with $\mathrm{CH}_{3}$ group, Masuko et al. also investigated effect of head groups on tribological performance of trichlorosilane SAMs on silicon. ${ }^{56}$ When a $\mathrm{Cl}$ atom was replaced an increase of COF was observed. Further increase of COF was shown when two $\mathrm{Cl}$ atoms were replaced. The authors concluded that replacing $\mathrm{Cl}$ atom of trichlorosilane with $\mathrm{CH}_{3}$ group increased steric hindrance, thereby making the formation of a dense monolayer difficult as well as the loss of cross linking between silicon atoms, which have an influential effect on stability and wear life of SAMs.

Other SAMs. DePalma also studied undecyltrichlorosilane (UTS) and (tridecafluoro-1,1,2,2-tetrahydrooct-1-yl) trichlorosilane (FHOTS) SAMs on silicon wafers, however, found that friction was higher than OTS SAMs in both cases, possibly due to the thicker films of $25 \AA$ produced by OTS SAMs as opposed to $15 \AA$ and $10 \AA$ A of UTS and FHOTS SAMs, respectively. ${ }^{163}$ Satyanarayana et al. also studied 3-aminopropyltrimethoxysilane (APTMS), however, APTMS SAMs did not show any lubricating properties. ${ }^{75}$ The increase in COF compared to a bare wafer is likely to be due to the hydrophilic terminal group producing a higher adhesive force which increases friction. However, Li et al. investigated tribological properties of 3-aminopropyltriethoxysilane (APTES) and phosphorylated APTES SAMs on silicon wafer using contact mode AFM under $20 \mathrm{nN}$ load at $10 \mathrm{~Hz}$ scan rate, and observed that the COF was reduced from approximately 0.08 on bare Si wafer to around 0.03 on both APTES SAM and phosphorylated APTES SAM. ${ }^{68}$ Kang et al. prepared a 6-(3-triethoxysilylpropylamino)1,3,5-triazine-2,4-dithiol monosodium (TES) SAM on silicon, and investigated its tribological performance using ball on disk with a $4 \mathrm{~mm}$ steel ball under a $0.098 \mathrm{~N}$ load. The authors reported a lower COF value of 0.12 compared to bare silicon which kept stable for $130 \mathrm{~s}$ or approximately 300 cycles. The reduction of friction on TES SAMs was claimed to be the van der Waals forces between the terminal groups on the ring structures. ${ }^{52}$

2.6.2 Multicomponent SAMs. Notable problems for monolayers in tribological systems involve high loads that remove the monolayer and therefore the lubricating qualities. Therefore, efforts have been made to improve the wear life of SAMs without losing their friction reducing capabilities. Two approaches have been implemented to achieve this, dualcomponent and multilayers. Dual-component SAMs use two different precursor molecules to form mixed monolayers where multilayers are produced through sequential steps to achieve the desired effect.

Booth et al. used OTS and (1-trichlorosilyl undecyl)trichloroacetate to create a dual-component gradient monolayer film on a silicon substrate. ${ }^{63}$ Dry sliding tests with a ball-onplate microtribometer demonstrated that these gradient SAM films exhibit excellent lubricating performance with significant reduction in friction in comparison to bare $\mathrm{Si}$ substrate. By creating dual-component monolayers with methyl and hydroxyl terminals, greater durability was achieved on the mixed SAM films with constant lubricating performance over $5 \mathrm{~h}$ at a sliding speed of $0.1 \mathrm{~mm} \mathrm{~s}^{-1}$. The enhancement in tribological performance durability was proposed to stronger intermolecular interactions within dual-component mixed SAMs. Singh et al. observed contrary effect on mixed SAMs of OTS/DTS and OTS/ HTS using microtribological tester with the deteriorated performance for both mixed SAMs compared to single component OTS SAM. ${ }^{\mathbf{1 6 6}}$ However, under nanotribological testing conditions using AFM, both mixed SAMs outperformed the single component SAMs, which was postulated that the difference in carbon chain length provided less resistance as the AFM tip slides over the outer layer.

Satyanarayana et al. also investigated tribological properties of a composite film of OTS onto APTMS SAM. The authors demonstrated that the composite film exhibits lower COF and longer wear life than APTMS SAM, but nearly the same performance as OTS SAM. This film lasted slightly fewer cycles at equivalent COF to that of the OTS SAM before breaking down at 3000 cycles. $^{75}$ Ma et al. created dual-layer films OTS/multiplyalkylated cyclopentane (MAC) and tested them using a ball on plate tribometer with a steel ball at a sliding speed of $1.5 \mathrm{~mm}$ $\mathrm{s}^{-1}$ under 0.5 and $1 \mathrm{~N}$ loads. OTS/MAC dual-layer films maintained a similar COF to OTS SAM through the $0.5 \mathrm{~N}$ testing but improved wear resistance through higher loaded tests. The improvement was attributed to that the MAC layer is able to reorganise and replenish the lubricant deprived area. ${ }^{\mathbf{7 0}, 108}$ Ren et al. prepared polyethyleneimine (PEI) SAM as well as a PEISTA dual-layer film on silicon substrate by immersing the PEI modified substrate in a solution of stearic acid (STA) and $N, N^{\prime}$ dicyclohexylcarbodiimide (DCCD). ${ }^{72}$ Using AFM and a unidirectional ball-on-plate tribometer, the authors demonstrated that the PEI SAM alone was not a good coating and only lasted a few cycles under low load. However, the PEI-STA dual-layer film shows much better tribological performance with a COF of 0.06 under the same load lasting for approximately 8600 cycles. The PEI SAM was not effective to reduce friction due to its minimal chain length and hydrophilic terminal group, while better performance of the PEI-STA film is possibly due to the long chain length of stearic acid which is able to rearrange the carbon chain in the shear direction and provides protection to wear. Yang et al. used (3-mercaptopropyl)trimethoxysilane (MPTS) to form a SAM on silicon followed by immersion in a silver nanoparticle solution resulting in a MPTS SAM doped with Ag. The doped layer was immersed in an octanethiol solution to form a sandwich-like trilayer film. Nanotribological testing performed using AFM under a load of $20 \mathrm{nN}$ demonstrated that compared with the bare Si wafer, MPTS SAM reduces the frictional force by 3.5 times, Ag-doped MPTS SAM further reduce by more than $40 \%$, and tri-layer film further reduces additional $15 \%$. The authors proposed that the long tails of the octanethiol are able to pivot and therefore rearrange with the sliding direction of the AFM tip reducing the frictional force. Using a reciprocal tribometer with a load of $0.5 \mathrm{~N}$ at a sliding rate of $2.5 \mathrm{~mm} \mathrm{~s}^{-1}$, it is observed that under the selected macro-sliding conditions, the MPTS SAM is not suitable for surface protection of Si substrate with high COF as bare Si and a short film life of 200 s. However, improvement was evident after the SAM was doped with $\mathrm{Ag}$ nanoparticles and 
additional layer. The silver doped MPTS SAM exhibited an extended antiwear life of $13000 \mathrm{~s}$ with a COF of 0.19 , while the trilayer film showed over doubled lifetime of $29500 \mathrm{~s}$ with a further reduction in COF of $0.16 .^{74}$

To summarise, the most successful SAMs have shown to be longer chain SAMs and multilayers. However, multilayers inherently have their own problems with application to tribological contacts apart from NEMS and MEMS. This area appears to be where research is focussed with SAMs proving their worth, notably OTS. The majority of SAMs reviewed in this article, single, mixed or multiple, have been application driven with regards to NEMS/MEMS with exceptions for DePalma's earlier work which was purely interested in the tribological properties.

\subsection{SAMs on silicon nitride}

2.7.1 Silicon nitride for bearings. Silicon nitride rolling element bearings have seen great success as hybrid bearing systems. Applications include automotive, aerospace, renewables and the railway industry. Ball bearings up to approximately $8 \mathrm{~mm}$ in diameter can be formed by cold isostatic pressing, compacting powder granulates by dry pressing or rolling granulation of powder nuclei. The larger ball bearings (diameter $>8 \mathrm{~mm}$ ) are typically formed by cold isostatic pressing. ${ }^{167}$ These silicon nitride bearing materials typically contain bindery additives such as $\mathrm{Fe}_{2} \mathrm{O}_{3}, \mathrm{Y}_{2} \mathrm{O}_{3}$ and $\mathrm{Al}_{2} \mathrm{O}_{3}$ depending on manufacturing procedure. ${ }^{17,149,167}$ All ceramic rolling element bearings can operate at temperatures up to 1000 ${ }^{\circ} \mathrm{C}$ with high chemical resistivity. The main sought after property is the relative lightness of the ball bearing, with a $60 \%$ reduction in weight resulting in an $80 \%$ reduction in friction compared to classic steel bearings. ${ }^{17}$ Compared to steel on steel contacts the COF of hybrid systems is reduced to approximately 0.04-0.09 under oil lubricated conditions and between 0.1-1.0 for dry conditions. ${ }^{149}$ Research shows that centrifugal loading on the outer bearing raceway is reduced by a lighter ball bearing, it has also been identified that ceramic bearings perform better under lubricant starvation and hard particle contamination. ${ }^{\mathbf{1 7}, \mathbf{1 9}, \mathbf{1 6 8 - 1 7 0}}$ Debris created by contact fatigue stresses can be suspended in the lubricant and create secondary wear mechanisms in which the suspended wear particles abrade, scratch and cut the surface creating further damage. ${ }^{149}$

2.7.2 Silicon nitride tribology. With a $2 \mathrm{~N}$ loaded reciprocated tribometer, Dante et al. studied the effect of protective oxide layer of silicon nitride on tribological properties without any surface modification, and found that the hydroxylated oxidised silicon layers $\left(\mathrm{Si}(\mathrm{OH})_{4}\right)$ produced a lower COF than pure silicon oxide $\left(\mathrm{SiO}_{2}\right) \cdot{ }^{171,172}$ The authors also found that at a temperature above $400{ }^{\circ} \mathrm{C}$ the silicon surface is dehydroxylated leading to a sustained high COF. Bal et al. also studied the tribological effect of silicon nitride and the protective oxidative layer and stated that the ability to re-oxidise after the oxidised layer has been removed can limit damage of the surface. The authors state that the two routes of degradation of silicon nitride are mechanical and tribochemical. The mechanical mode occurs under high loads and low speeds with frequent stop-start conditions. The tribochemical wear is when silicon nitride reacts with water initially forming silicon dioxide and ammonia followed by the silicon dioxide reacting with more water to produce silanol groups. ${ }^{171-175}$

2.7.3 SAMs for silicon nitride. Details of the crystal structure of silicon nitride can be found in ref. 176 and 177. A thin oxide layer (2-4 nm) is naturally found on the surface of silicon nitride and allows SAM attachment in the similar way as on silicon wafers. ${ }^{\mathbf{1 7}, \mathbf{1 7 1 - 1 7 3 , 1 7 8 - 1 8 0}}$ The oxide layer on silicon is amorphous silicon oxide, conversely there is an interface layer of silicon oxynitride that is present on silicon nitride as shown in Fig. 11, ${ }^{177}$ which has been confirmed through TEM and XPS. Hydroxylation of silicon nitride can be achieved by plasma and piranha treatment. ${ }^{180,181}$

Sung et al. showed that monolayers of octadecyldimethylchlorosilane (ODS) could be formed on silicon nitride. ${ }^{\mathbf{1 8 2}}$ After treating with $\mathrm{HF}$, the monolayers were formed on silicon nitride from a solution of ODS in mixed solvents of hexadecane :chloroform $(4: 1)$ for one hour. Water contact angle of $110^{\circ}$ are obtained on the ODS SAM modified silicon surfaces, which are similar to those reported for silicon oxide, showing a good monolayer was formed on silicon nitride surface. ${ }^{182,183}$ This study also demonstrated that the monolayers can attach directly to H-terminated silicon nitride as well as the naturally found silicon oxide. With piranha treated silicon nitride good quality SAMs can also be formed on the oxide layer, as Kölbel et al. demonstrated the SAMs of chlorosilanes and ethoxysilanes formed on the piranha-treated silicon nitride exhibited equivalent contact angles to those SAMs formed on silicon wafers. ${ }^{\mathbf{1 8 4}}$ Stability testing by washing with solvent, storing in water, heating or storing in ambient conditions for months did not affect the contact angle. ${ }^{\mathbf{1 8 4}}$ Diao et al. successfully produced monolayers of APTES on silicon nitride with contact angles of $\pm 5^{\circ}$ comparable to that obtained on silicon wafers by Janssen et al. ${ }^{\mathbf{1 0 3 , 1 8 5}}$ Wang et al. investigated SAM formation on silicon nitride using four silanes; OTS, octyltrichlorosilane, chlorodimethyloctadecylsilane and octadecyltrimethoxysilane. ${ }^{\mathbf{1 7}}$ It
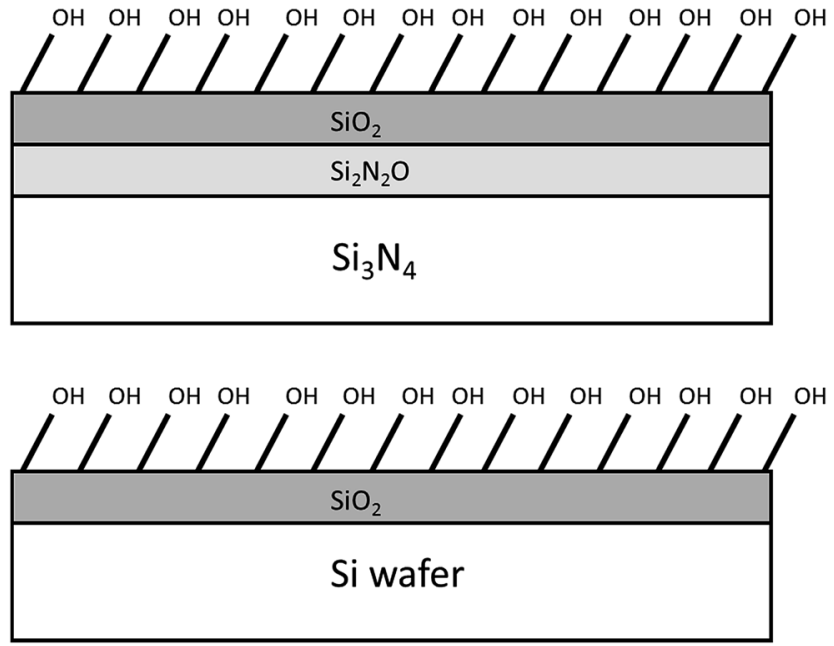

Fig. 11 Detailing the oxide layer present on both Si wafers and silicon nitride. 
was found that OTS and octadecyltrimethoxysilane achieved the highest contact angle of $108^{\circ}$. Like Kulkarni et al., the authors found that the initial formation was very quick and $80-90 \%$ of the monolayer is formed within an order of minutes. ${ }^{31}$

2.7.4 SAM formation on silicon nitride and tribological applications. To further improve the performance of silicon nitride in tribological applications, feasibilities of SAM formation on silicon nitride and their tribological performance has been investigated in recent years. The following section has been divided into macro and nanotribology of SAMs on silicon nitride surfaces, where the nanotribology focuses on the modification of silicon nitride cantilevers in AFM.

Nanotribology. The majority of AFM tips are made of silicon nitride with pyramidal contact. SAMs have been applied to AFM tips to create chemical force microscopy (CFM) ${ }^{\mathbf{1 8 6}}$ which is used to measure frictional response between the cantilever modification and substrate. The modified tip is useful for mapping different chemical functionalities across a substrate through the surface-tip interactions as some SAMs will result in different frictional responses. In some cases the cantilevers are first coated in gold then thiols are adsorbed, silanes have also been reported. ${ }^{\mathbf{1 8 7}}$ The gold layer can be detrimental to friction experiments as the gold is weakly attached to the silicon nitride so for nanotribology direct attachment to silicon nitride is preferable. ${ }^{187}$ Adhesion testing is common with CFM, it can simply be explained as the force needed to retract the cantilever from the surface. Adhesion force measurements are related to the modified tip and surface interactions and can be used to measure stiction and combat adhesion related failures notable in NEMS/MEMS. ${ }^{188-190}$ Direct comparison of adhesive forces can be misleading if different tip radii are present. ${ }^{187}$ This is even more important if colloidal AFM cantilevers are used. ${ }^{191}$ Ito et al. found that adhesion force of CFM probes is in agreement with the increase of contact angle of the same SAM on a planar silicon nitride substrate. ${ }^{192}$ Headrick et al. shows an increase in pull off force when OTS SAM are applied to silicon nitride cantilever on $\mathrm{a}_{3} \mathrm{CH}_{3}$ terminated substrate. ${ }^{193}$ OTS SAM cantilevers on $\mathrm{COOH}$ terminated surfaces show a slight increase in adhesion force in comparison to cleaved mica, this gives the unique ability to identify localised chemical groups on the surface through CFM through differences in pull off forces. ${ }^{193}$ Tsukruk et al. modified both cantilever and substrate and found that larger force displacement curves are recorded with $\mathrm{CH}_{3}$ $\mathrm{CH}_{3}$ terminations rather than $\mathrm{Si}_{3} \mathrm{~N}_{4}-\mathrm{Si}_{3} \mathrm{~N}_{4}$ contacts. ${ }^{187}$ Frisbie et al. modified gold coated silicon nitride cantilevers with both $\mathrm{COOH}$ and $\mathrm{CH}_{3}$ terminating SAMs. ${ }^{194}$ Fig. 12A shows a representation of the lithographically defined SAMs. Fig. 12B and C show the frictional response of a $\mathrm{CH} 3$ terminated cantilever and a $\mathrm{COOH}$ cantilever, respectively. Brighter areas are areas of higher friction.

Macrotribology. An extensive literature search resulted in very little with regards to silicon nitride modified with SAMs. The authors found one publication reporting the results on SAMs on silicon nitride at macro scale. Wang et al. investigated SAM the tribological properties of two SAMs, OTS and octadecyltrimethoxysilane on silicon nitride. ${ }^{17}$ OTS is discussed here as it was a superior friction reducer. However, as the authors also pointed out, although octadecyltrimethoxysilane is not as good in reducing the COF as OTS, it may be more appropriate to use it instead of OTS as an additive for hybrid contacts, as there are fewer corrosive by-products than OTS (see eqn (1) and (2)). Tribological testing was conducted using a ball on disk tribometer, where the COF of a SAM modified disk was compared to that of an unmodified disk under a $10 \mathrm{~N}$ load and at a constant speed of $0.198 \mathrm{~m} \mathrm{~s}^{-1}$. Under dry conditions the OTS SAM produced a COF of 0.020 for $11.6 \mathrm{~m}$ before failure. When lubricated with a base oil the COF is reduced to 0.014. Interestingly the authors discovered that the best COF result comes from using OTS as an additive in a base oil, at concentrations of 2.5, 5 and $10 \mathrm{mM}$ were tested. After $1250 \mathrm{~m}$ sliding the COF has settled to a steady value of 0.008 for all three solutions.

\subsection{SAMs on metallic substrates and tribo-applications}

Apart from on silicon wafer and silicon nitride, SAMs have also been developed on many other substrates such as alumina, iron oxide, steel, zinc, copper, platinum, silver, $\mathrm{Ti}_{6} \mathrm{Al}_{4} \mathrm{~V}$ alloy, as well as gold, palladium, silver, copper and zinc. However their applications in tribology are still limited. ${ }^{91}$ This section reviews the development SAMs on metallic surfaces based on the head groups of the SAMs.

2.8.1 Silane head groups. OTS is the most common head group that has been investigated on various metal surfaces. Zhu et al. developed a novel method of constructing OTS monolayers on stainless steel to reduce the corrosive effects of hydrochloric acid by-products which could also be related to silicon nitride. The extended storage of OTS in solvent increased levels of hydrolysis, this could then be neutralised to reduce the aforementioned corrosive effects (see eqn (1) and (2)). This method did not hinder the ability of the monolayer to form on the substrate and water contact angles of approximately $108^{\circ}$ were regularly achieved, similar to that of silicon and silicon nitride. OTS monolayer formation was facilitated by a highly hydroxylated surface. ${ }^{145}$

OTS has also been attached to amorphous alumina and the kinetics as well as resulting monolayer are shown to be very similar to that of a silicon oxide substrate. Facilitated by hydroxylated surfaces SAM growth occurs with reported contact angles of $100^{\circ}$. However, the authors did not achieve known contact angles with Qin et al. and Wang et al. achieving considerably higher $\approx 120^{\circ} .^{\mathbf{1 9 5}, 196}$

Qin et al. investigated the performance of octadecyltriethoxysilane, $\quad 1 \mathrm{H}, 1 \mathrm{H}, 2 \mathrm{H}, 2 \mathrm{H}$-perfluorodecyltriethoxysilane (PFDS) and APTES monolayers on aluminium alloys ${ }^{117}$ using a reciprocating ball on plate test rig. A steel ball was used as the counter surface. APTES failed instantaneously possibly due to short chain length not preventing contact but also due to the head group. PFDS successfully reduced the COF under loads of $0.6,1$ and $2 \mathrm{~N}$ for sliding times of 600 seconds, but failure occurred at approximately 400 seconds under $3 \mathrm{~N}$ load. Octadecyltriethoxysilane reduced the COF the most and completed all of the tests as well as a $4 \mathrm{~N}$ test without failure, the authors concluded the same mechanism for lubricity exists on aluminium as it does on silicon. The long chain coupled with 

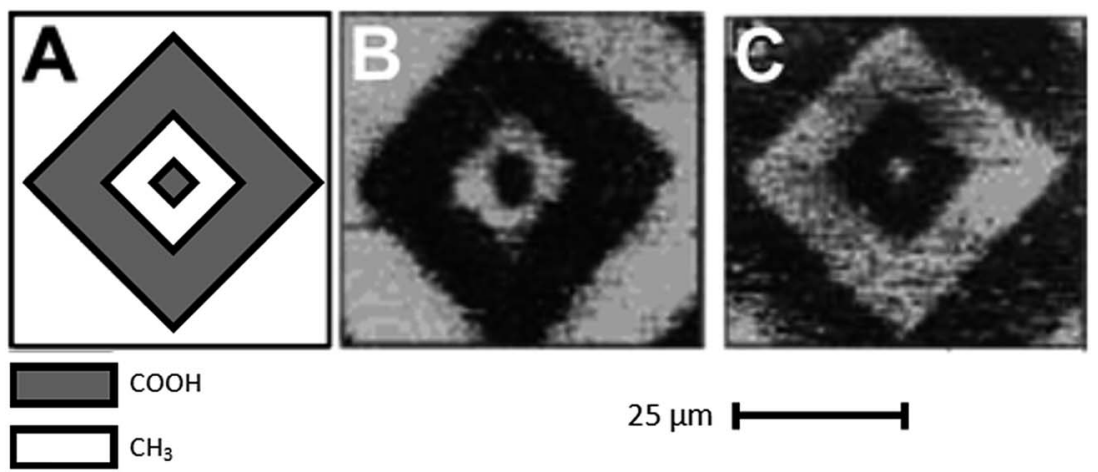

Fig. 12 Representations of frictional force between a modified substrate shown in (A). (B) Is where a $\mathrm{CH}_{3}$ terminated cantilever is used and (C) is with a $\mathrm{COOH}$ terminated tip. Brightness is related to areas of higher friction. Adapted from Frisbie et al. and Barattin et al. Reprinted with permission from ref. 194.

the ability to swing as well as inter-chain interactions all help to reduce COF. ${ }^{117}$ Similar results were achieved by Devaprakasam et al. when $1 H, 1 H, 2 H, 2 H$-perfluorooctadecyltrichlorosilane (FOTS) and OTS were formed on aluminium and tested on both nano-and macrotribometers. ${ }^{94}$

Panjwani et al. formed 3-glycidoxypropyltrimethoxysilane on $\mathrm{Ti}_{6} \mathrm{Al}_{4} \mathrm{~V}$ alloy, and then coated a perfluoropolyether (PFPE) layer on top of the preformed SAM. They tested the treated alloy against a silicon nitride ball on a ball on disk tribometer under $0.2 \mathrm{~N}, 41.9 \mathrm{~mm} \mathrm{~s}^{-1}$ and dry sliding conditions. It was found that the PFPE overcoat lasted over 900 times longer than without the coat due to its self-repairing characteristics. ${ }^{197} \mathrm{Li}$ et al. created an APTES SAM on titanium alloy followed by a self-assembling graphene oxide (GO) layer as illustrated in Fig. $13 .{ }^{198,199}$ Reduced graphene oxide (RGO) sheets are prepared by heating GO sheets after assembly.

The modified Ti surfaces were then tested using a reciprocating pin on plate tribometer under dry conditions against a silicon nitride ball under $100 \mathrm{mN}$ load and $1 \mathrm{~Hz}$ frequency. The APTES SAM showed a poor wear life and was destroyed within 500 seconds. The graphene oxide/APTES film however showed a COF of 0.19 and a life time of over 5000 seconds. This was further improved after heat treatment, where a COF of 0.16 and a life of 12000 seconds were achieved. It was suggested that it was due to less oxygen groups in the heat treated graphene layer. ${ }^{199}$
2.8.2 Other head groups. Other head groups have been researched, notably carboxylic and phosphate. Carboxylic acids have been and still are used as friction modifiers and it is thought that they can form monolayers on surfaces. ${ }^{200,201}$ Carboxylic acids have been found to spontaneously adsorb on to numerous substrates, such as $\mathrm{AgO}, \mathrm{CuO}$ and $\mathrm{Al}_{2} \mathrm{O}_{3}$. However, there is a difference in how the monolayers adsorb onto surfaces, Fig. 14A and B shows the different methods. On AgO, for example, it has been found that the carboxylate binds symmetrically to the substrate unlike $\mathrm{CuO}$ which follows $\mathrm{B}$. Fig. 14C shows the bonding characteristics of alkylphosphonic acid on a substrate, this is dependent on the substrate properties. ${ }^{202}$ Some examples are reviewed below. ${ }^{17,18}$

Wan et al. created SAM of alkylphosphonic acids on copper surfaces, where dodecylphosphonic (C12PA) and octadecylphosphonic (C18PA) acids were chosen. Ball on plate reciprocating tests with $5 \mathrm{~mm}$ steel balls under a load of $0.5 \mathrm{~N}$ and sliding velocity of $10 \mathrm{~mm} \mathrm{~s}^{-1}$ were performed. The C12PA SAM remains stable for approximately 50 seconds before failure and the C18PA SAM lasts more than 500 seconds before slow failure. This follows the same rules as silanes with regards to length of chain providing increased lubricity and wear life. Etching the copper surface using $\mathrm{NaOH}$ before SAM formation greatly improved the wear life of the films, wear life of the C18PA SAM formed on the etched copper substrate exceeded 7200 seconds under a COF of 0.2. Etching also increases the contact angle of

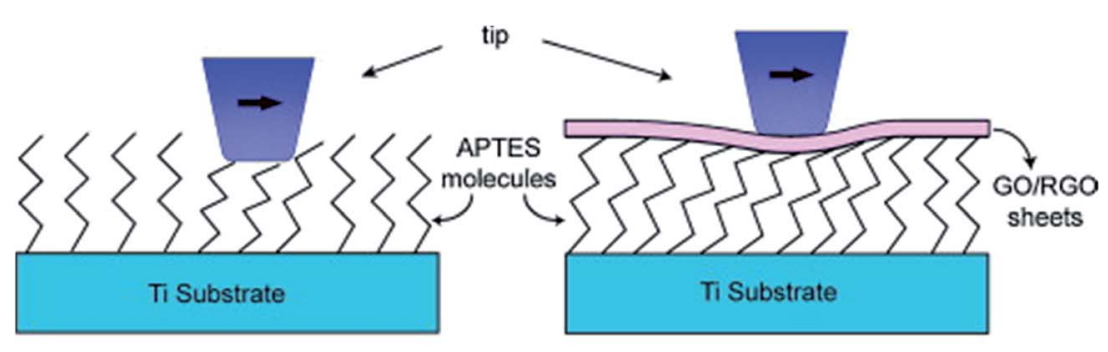

(a)

(b)

Fig. 13 Showing the interaction between the pin and APTES film and the ideal interaction with the GO/RGO sheets. Reprinted with permission from ref. 199. 


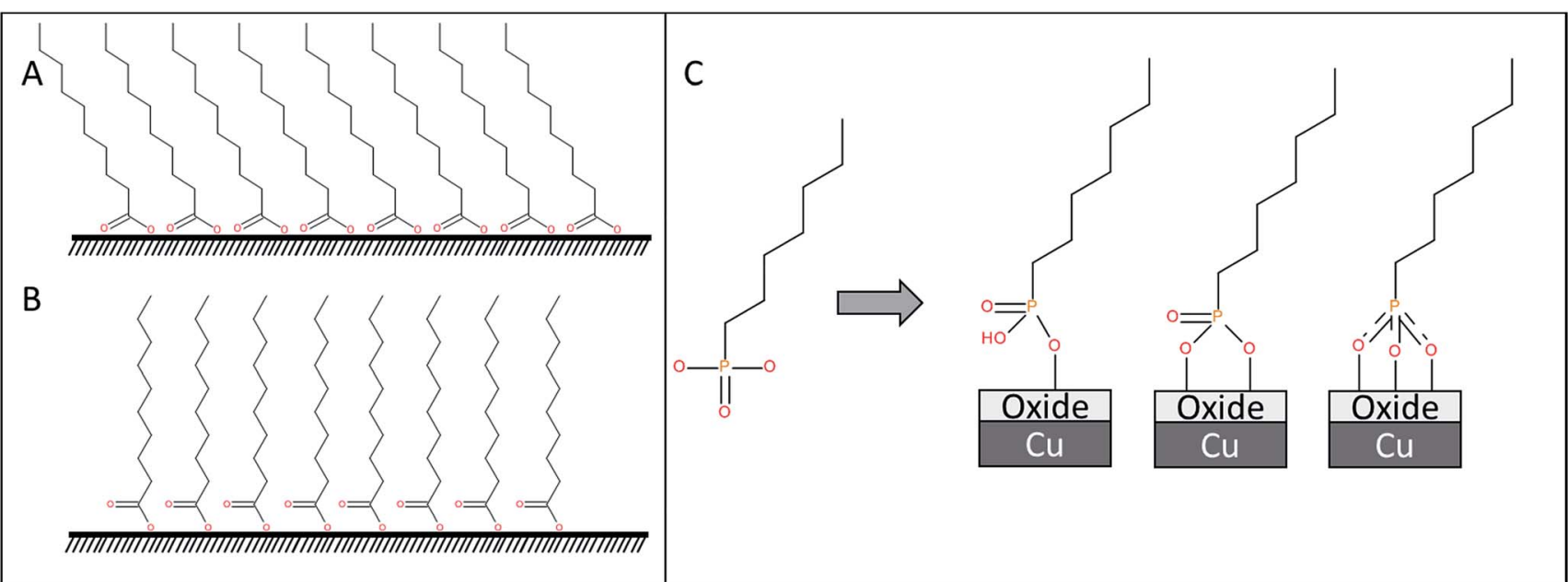

Fig. 14 Illustrations of the formation of carboxylic acid monolayers (A and B) and the bonding characteristics of alkylphosphonic acid on copper oxide. Information from ref. 200 and 202.

both C12PA and C18PA SAMs significantly which may play a part in friction reducing. ${ }^{202}$ The tribological application of both C12Pa and C18PA on alumina was also investigated by using a reciprocating ball-on-flat microtribometer under dry conditions. In addition it is shown that both acids can reduce the COF, however, the longer chain length reduced friction more which is consistent with the literature. ${ }^{148}$

Zhang et al. investigated stearic acid (SA) SAM films on textured aluminium using a ball-on-disk tribometer with steel ball counterparts dry sliding at $10 \mathrm{~mm} \mathrm{~s}^{-1}$ under $0.1 \mathrm{~N}$ load. Surface texturing was completed using $\mathrm{NaOH}$ at $100^{\circ}$ for $1 \mathrm{~h}$, etching alone reduced the COF to below 0.6 from over 0.7 for non-etched Al surface. SA SAM on non-etched Al substrates reduced the COF to below 0.5, while the SAM on the etched substrates the COF was further reduced to under 0.15 . The authors cited pivoting and rearrangement of the monolayer as an explanation for the reduction of $\mathrm{COF}^{203}$ As Wan et al. observed, ${ }^{202}$ Zhang et al. also concluded that SAM modification combined with chemical etching can significantly reduce friction with drastically increased durability, and low friction of the combined modification is due to the reduction in surface adhesion between steel ball and the films as well as the actual contact areas. ${ }^{203}$ Carboxylic acids have been used to lubricate steel and diamond like carbon (DLC), in research completed by Simič et al. palmitic acid was the acid of choice. In the tribological tests palmitic acid was premixed into a poly alpha olefin base oil, testing was performed using a ball on flat reciprocating tribometer with steel on steel contacts or DLC on DLC at $25^{\circ} \mathrm{C}$ and $80^{\circ} \mathrm{C}$ with a load of $10 \mathrm{~N}$ at an average velocity of $0.01 \mathrm{~m} \mathrm{~s}^{-1}$. Steel on steel testing at the lower temperature shows that the COF is reduced from approximately 0.15 to 0.10 for the premixed oils, at higher temperatures the reduction effect is increased attributed to the greater mobility of the additive palmitic acid. However, palmitic acid showed no effect on friction reduction for the DLC-DLC contacts at $25{ }^{\circ} \mathrm{C}$ and a slightly negative effect at $80{ }^{\circ} \mathrm{C} .{ }^{204}$

\subsection{Challenges of SAM for lubrication}

The literature shows that the uniformity and hydrophobicity of SAMs are critical to friction reduction of the SAM coated silicon substrates. This has been shown above by head group selection and limited adsorption before tribological testing. Another factor is chain length, many of the studies mentioned have compared the difference and it is regularly reported that longer i.e. 18 carbons (OTS), performs best under a variety of tribological conditions. This appears to be valid over a range of substrates. This ability to reduce friction is commonly related to the ability to rearrange under shear stress with longer chains able to withstand compressive forces better. However, the durability of the SAMs is still a concern for practical applications as ultra-thin single SAMs easily break down under high loads or exhibit limited wear life. It has also been noted that high pressures and temperatures could remove monolayers. ${ }^{94}$ Fig. 15 shows that OTS can perform well under low loads in comparison to a bare silicon wafer (A and B), however under higher loads the monolayer will fail. That said, there is still a reduction in wear indicating that the OTS SAM did not fail instantaneously (C and D).

Shear stress is a limiting factor of SAM lubrication, therefore research has focussed on forming composite SAM films or SAM multilayers. Multilayers, such as MAC, have shown promising results by improving the wear life of OTS SAM. These methods require different formation techniques such as extended spin coating or thermal annealing, for some applications such as in situ bearing lubrication this may not be feasible. This of course presents its own difficulties not just involving costs and maintenance but condition monitoring of lubricants. However, preformed SAM multilayers formed before use may prove feasible for applications. As there is no set testing standards the nature of investigations is inherently scattered. This obviously means that it is difficult to comprehend all the findings of a wide variety different contact size, geometries, load, speed etc. 


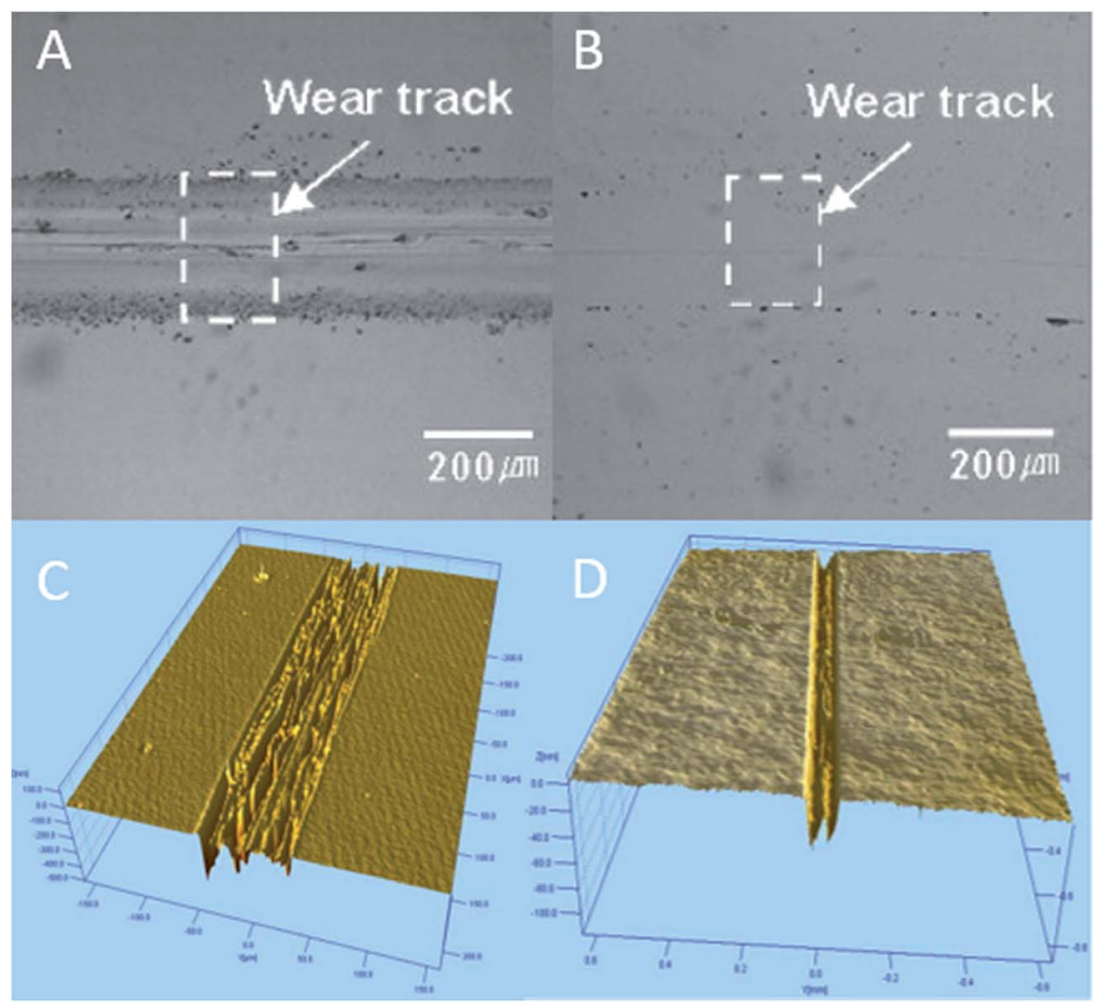

Fig. 15 Comparison of the wear tracks of a silicon wafer (A) compared to an OTS SAM (B) that has not failed. (D) Shows the wear track of an OTS SAM that has failed in comparison to a bare silicon wafer (C). (C and D) Reprinted with permission from ref. 70 . (A and B) Reprinted with permission from ref. 162

\section{Polymer brushes}

Whilst SAMs are advantageous because of their simple formation, e.g. thiols on gold and chlorosilanes on oxides, and the end groups are easily modifiable for specific functions. Their application as a lubricant in tribological contacts is limited as thin film SAMs are susceptible to shearing..$^{25,26}$ In a similar way, polymer brushes are formed by adsorption of prefabricated polymers onto the substrate through their reactive function groups or by subsequent polymerization onto reactive-terminal group containing SAMs. Polymer brushes typically have longer carbon chain and better durability than SAMs, thus better capabilities of polymer brushes to withstand compressive force and shear stress are expected for a better lubrication solution. Polymer brushes have been used to change wettability, reduce friction and increase corrosion resistance by using a variety of polymers. $^{205-210}$ This section reviews the development of polymer brushes and their application in tribology systems.

Polymer brushes are formed onto surfaces through two methods, named 'grafting to' and 'grafting from' processes. The 'grafting to' method uses prefabricated polymers that can be attached to a surface using physisorption or chemisorption much like SAM as seen in the left route of Fig. 16. The film thicknesses of polymers produced through the 'grafting to' method are limited by the molecular weights of the preformed polymer in solution. ${ }^{210,211}$ Although this method is relatively easy to carry out as it works much like a SAM, there is steric hindrance that impedes the density of the final film that is formed..$^{71,212,213}$ In addition to this, the adsorption techniques are reversible so that the layers may be susceptible to high shear forces. $^{210}$

In the 'grafting from' techniques, also known as surface initiated polymerisations, the surface is first modified with a self-assembling initiator layer, which is then exposed to monomeric components with catalyst and, if needed, in an appropriate solvent. ${ }^{\mathbf{2 1 0 , 2 1 4 - 2 1 6}}$ This can be seen in the right route of Fig. 16. It is imperative that the substrate to be modified has successfully had initiator molecules anchored to it, otherwise the solvent would become gelatinous due to polymerisation taking place. This in turn would then affect the density of the polymer film by way of steric hindrance. ${ }^{210}$ This method allows much more control over the final film and the grafting densities can approach 1 chain per $\mathrm{nm}^{2}$ (ref. 208, 211, 217-219) compared to the $0.05-0.1$ chain per $\mathrm{nm}^{2}$ for 'grafting to' strategies. ${ }^{\mathbf{1 2 1 , 2 2 0}}$ The improved density is due to the less steric hindrance from long chains to the substrate. In comparison to the limit of the films using 'grafted to' methods (<100 nm thickness), the 'grafting from' method can produce much thicker films. ${ }^{210}$

\subsection{Radical polymerisation}

There are many different polymerisation reactions that can be used to initiate polymer brushes, such as ring opening metathesis polymerisations, ${ }^{\mathbf{1 8 1 , 2 1 0 , 2 1 1 , 2 2 1}}$ nitroxide mediated 


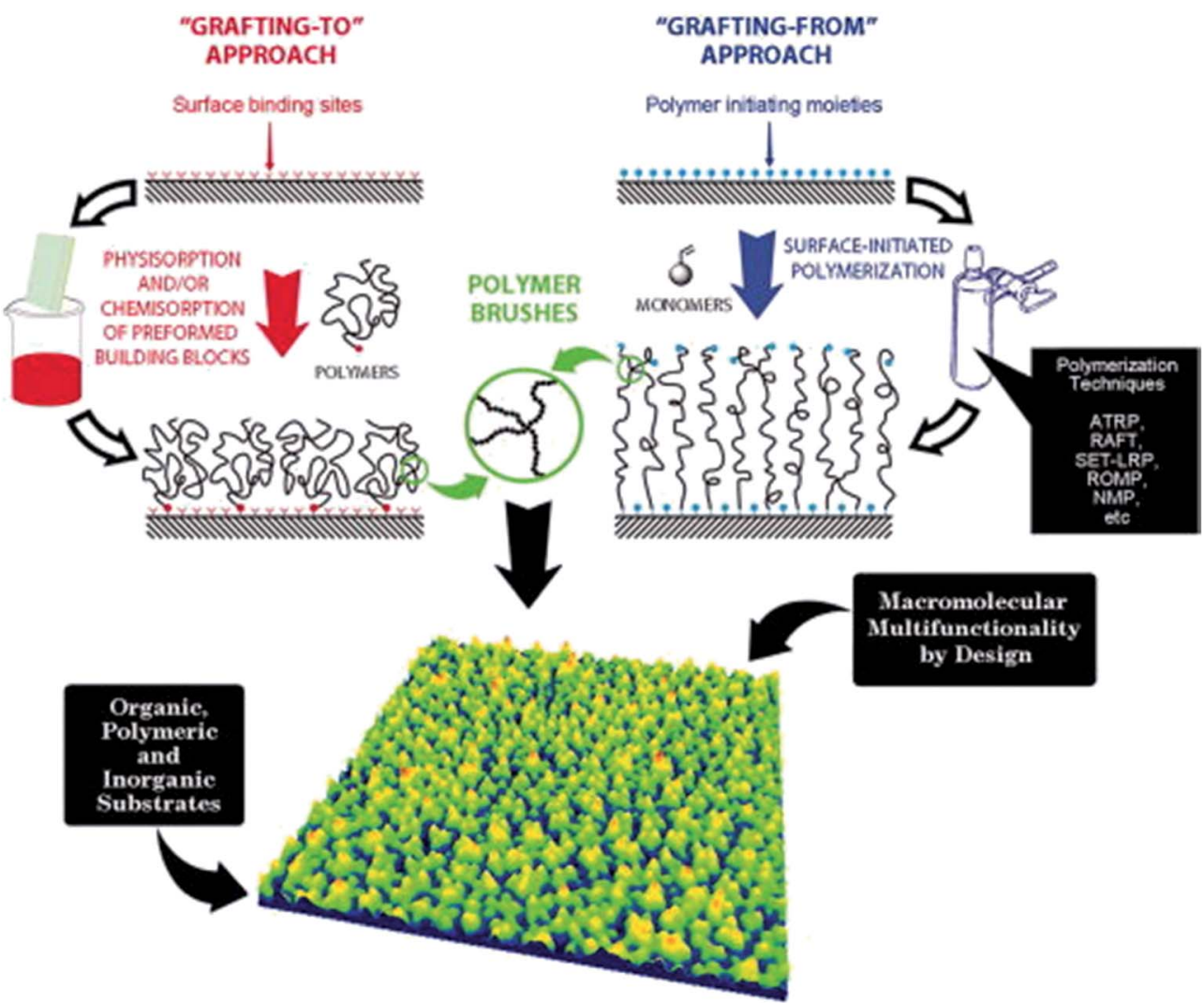

Fig. 16 Schematics of the two different schemes of polymer brush formation and highlighting the density differences. The scheme also includes an AFM image showing $\left(500 \times 500 \mathrm{~nm}^{2}\right)$ of poly (2-(methacryloyloxy)-ethyl-trimethyl-ammonium chloride) brushes grown from a Si wafer via surface initiated ATRP. Reprinted with permission from ref. 205.

polymerisations, ${ }^{210,211,221,222}$ reversible addition fragmentation chain transfer polymerisation, ${ }^{181,210,211,221,222}$ atom transfer radical polymerisation,,$^{3,27,181,210-212,214,221-227}$ and living ring opening polymerisations. ${ }^{181,210,221}$ As ATRP is covered in depth its entirety is discussed in 3.2 Atom transfer radical polymerisation.

3.1.1 Ring opening metathesis polymerisation. Ring opening metathesis polymerisation (ROMP) is a subsidiary of ring opening polymerisations (ROP) which is focussed on the breaking of carbon-carbon double bonds. ${ }^{228}$ ROMP is a form of chain growth polymerisation where cyclic monomers produce straight or fewer ringed additions to the polymer chain. ${ }^{229}$ Notable applications of ROP are the production of nylon 6 from caprolactam avoiding the patented route of polycondensation. ${ }^{230}$ ROP of lactams has been investigated for over 40 years due to the ability to produce a variety of polymers in a controlled manner. ${ }^{221}$ Although surface initiated polymerisations are possible the focus has been on coating grafted polyesters. ${ }^{21}$ Ring opening metathesis polymerisations are a variation of this procedure in which unsaturated monomeric components are polymerised but still contain unsaturated components. $^{229}$ The driving force of ROMP is ring strain enthalpy. ${ }^{228,231}$ Applications include rubber additives and super adsorbent materials. ${ }^{228}$
3.1.2 Nitroxide mediated polymerisation (NMP). Nitroxide mediated polymerisations (NMP) are based upon activation/ deactivation of a chain end radical with a nitroxide leaving group. ${ }^{21,222}$ NMP provides a good route for polymer brush construction without the need for catalysts, but still able to produce controlled polymers in a narrow molecular weight range. ${ }^{222}$ Due to the simplicity of NMP and the ability to use alkoxyamines as initiating and mediating species applications have increased. ${ }^{232}$ Polymerisation opportunities completed in solution have been increased due to the development of a universal alkoxyamines which has also resulted in the ease of use of NMP. ${ }^{233}$

3.1.3 Reverse addition fragmentation. Reversible addition fragmentation chain transfer polymerisation is a different type of controlled radical polymerisation by using chain transfer agents such as thiocarbonylthio compounds. ${ }^{221}$ It is commonly initiated by azobisisobutyronitrile (AIBN) that decomposes to form a radical, reacts with a monomeric unit to start polymerisation. To deactivate the chain, the polymer radical reacts with the chain transfer agent to create a reversible additionfragmentation chain transfer (RAFT) radical adduct. The RAFT radical adduct can then fragment and lose either the radical polymer or the 'R' group. ${ }^{234}$ The success of RAFT polymerisation depends on the ' $R$ ' group, which is a radical. This allows the initiation of another monomer and the start of another active 
chain. RAFT polymerisations are capable of achieving very narrow polydispersities, e.g. lower than 1.1, which is one of its advantages compared to other methods. ${ }^{235}$ Approaching equilibrium between the propagating radicals; one from the original interaction with AIBN or similar and the other from the ' $R$ ' group radical and monomer, and the deactivated chain, means that all the chains have the same probability of growing, resulting in narrow polydispersity index (PDI). ${ }^{236}$ In addition, with regulations to reduce the amount of sulphur in lubricants, RAFT polymerisations may not be a viable option as chain transfer agents for RAFT usually contain sulphur element. ${ }^{17,237}$

\subsection{Atom transfer radical polymerisation}

ATRP is the most popular type of polymerisation for brushes due to the relative robustness of the technique. For example, unlike the other techniques, rigorously dry working conditions are not needed and reactions are very tolerant of a variety of monomers, ligands and catalysts. The commercial success of ATRP has been well reported, in part due to the polymerisations ability to be completed in commercially available equipment. ${ }^{210,213,221,227,238}$ ATRP can form well-defined polymer brushes from easily synthesised initiators and is well known as a successful controllable polymerisation. ${ }^{211}$

ATRP was independently developed by Matyjaszewski and Sawamoto initially for controlled radical polymerisations ${ }^{239,240}$ and has been used to efficiently produce many different polymers $^{223,224,241-243}$ due to its effective control in the composition and architecture of polymers. Surface initiated ATRP (SI-ATRP) was first reported by Huang et al. who used benzyl chloride attached to a trichlorosilane head to form a monolayer on silicon surface then poly acrylamide brushes using copper(I) chloride and bipyridine with acrylamide to create new stationary phases for chromatography. ${ }^{\mathbf{2 2 2 , 2 4 4}}$ Copper is usually the transition metal choice for ATRP. 27,121,222,241-243,245-247 Through ATRP, Ejaz et al. synthesised methyl methacrylate brushes on silicon wafers using a $\mathrm{Cu}$ based catalyst after a trimethoxysilane initiator was immobilised on the surface using LangmuirBlodgett techniques. ${ }^{\text {222,224,245 }}$ Since then the technique has been implemented onto gold, inorganic particles, organic latexes, formed dendrimers, highly functionalised linear brushes, and varied compositions and sizes. ${ }^{224}$

The basic structure for ATRP synthesis of polymer brushes is to have an initiator or monomer, and a catalyst made of a transition metal for successful grafting of chains. If the polymer brushes do not all grow at the same rate or time, the shorter chains can be inhibited from growing any further due to steric hindrance. This can be overcome by ensuring there are copious amounts of initiator sites on the substrate. There are two major steps in this polymerisation reaction, namely activation and deactivation. During the activation step, the metal complex breaks the alkyl halogen bond in the initiator, resulting in the formation of radicals. The radicals then propagate with the excess monomer and higher oxidation state metal complex. In the deactivation step, the radicals react with deactivators (e.g. polymer chain or activators) resulting in the formation of halide capped chains or reformed metal complex catalysts. For this to be successful, it is necessary to have a reversible reaction shifted to the dormant species, accompanied by fast initiation and deactivation. This is important as it can reduce the amount of termination steps. ${ }^{223,241}$ Due to the importance of ATRP in polymer brush development, it is further reviewed in the next section.

3.2.1 Initiators and synthesis. Initiators are key to ATRP, the ability to attach polymers to surfaces has been discussed with respect to grafting "to" and "from", however, to increase densities tethering the initiator is required. ATRP uses simple initiators that usually contain one or more halides. ${ }^{211}$ The architecture of the polymers can be varied by the initiator, to produce linear chains of polymers that only one halide is present. Using multiple halide systems can form other shapes, such as stars or combs. ${ }^{241,248,249}$ Development of self-assembling initiators for surface initiated polymerisations bridges SAMs into polymer growth. ${ }^{221}$ Much like SAM, for lubricating films, polymer brushes must be strongly attached to the contact surface, therefore, chlorosilane initiators are often chosen to be anchored to substrates in ATRP in the same way that SAM forms as shown in Scheme $1 .^{250}$ Ohno et al. also synthesized triethoxysilane initiators. ${ }^{251}$ Initiators do not have to be anchored to a substrate. In some cases free initiator is used to control the ATRP reaction where gel permeation chromatography (GPC) can be used to calculate polymer conversions. Details are given in the next section.

Liu and co-workers used the ATRP silane initiator (11'-trichlorosilylundecyl)-2-bromo-2-methylpropionate in their polymer brush formation. ${ }^{242}$ The silane initiator was synthesised using the esterification route detailed by Matyjaszewski, ${ }^{252}$ followed by hydrosilylation using trichlorosilane and Karstedt's catalyst as shown in Scheme 2. The authors also refer to the work of Husseman et al. for this synthesis. Husseman however, used Speier's catalyst instead. ${ }^{253}$ Karstedt's catalyst is more reactive requiring much lower amounts of Pt, Speier's catalyst is viewed as economically unattractive due to reactivity and that large amount of catalyst are rendered useless and unrecoverable. ${ }^{254}$ Bielecki et al. followed a very similar route except using

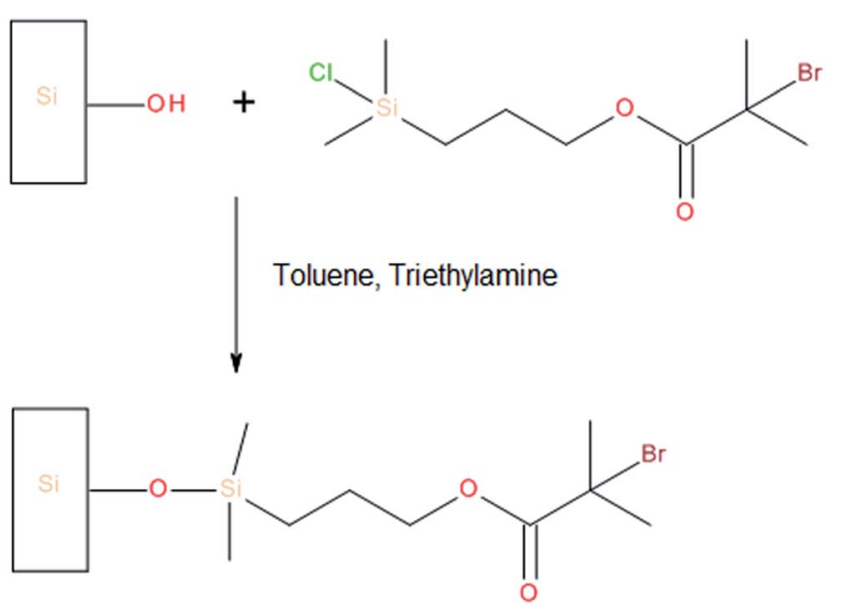

Scheme 1 Shows SI-ATRP, the attachment of the ATRP initiator (3-(2bromoisobutyryl)propyl)dimethylchlorosilane to a silicon wafer. 


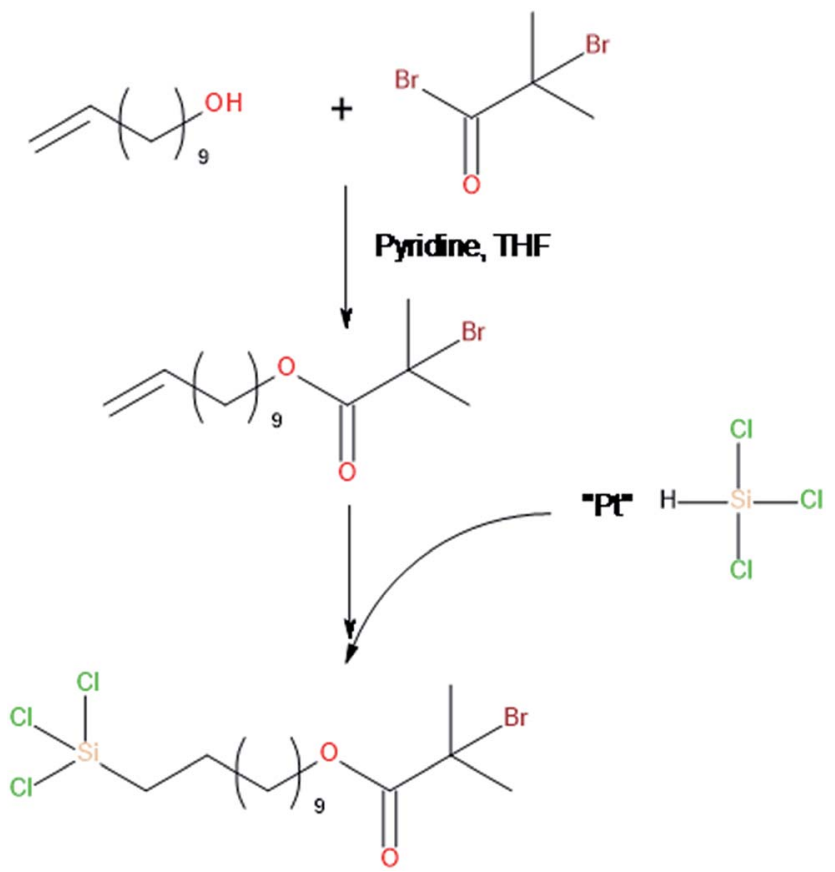

Scheme 2 Shows the synthesis of the ATRP initiator $\left(11^{\prime}\right.$-trichlorosilylundecyl)-2-bromo-2-methylpropanoate via esterification and hydrosilylation.

chlorosilane which produced a less reactive head group as shown in Scheme 3.

Zhou et al. constructed an initiator monolayer on silica particles, then APTES self-assembled over the following 9 hours to produce an amide functionalised end group. This was then

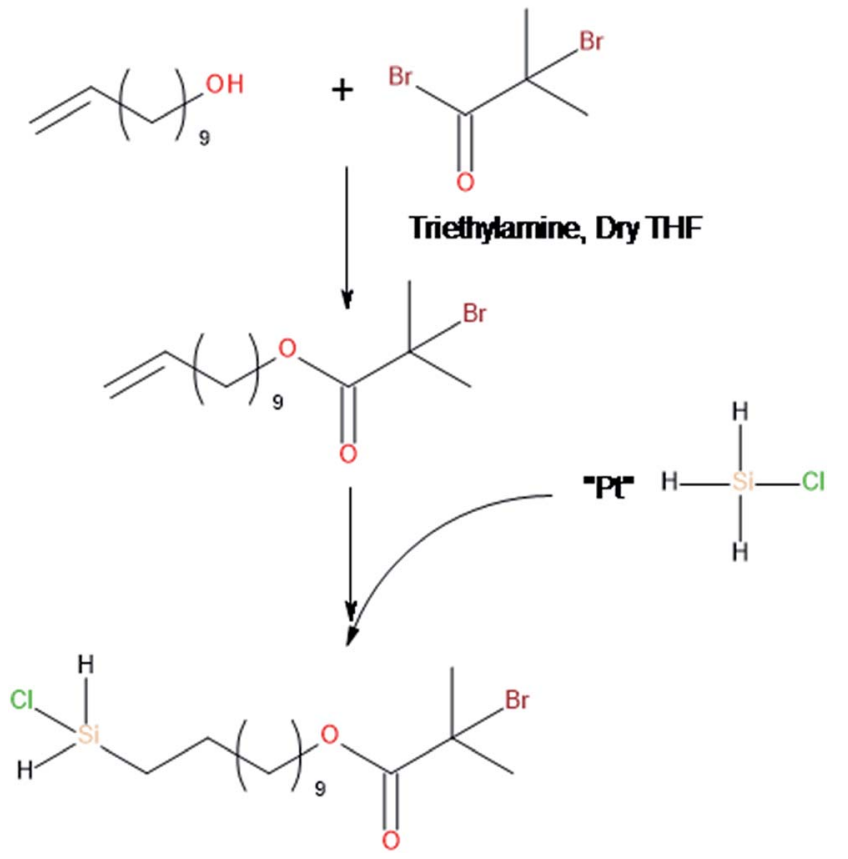

Scheme 3 Detailing the ATRP initiator (11'-chlorosilylundecyl)-2bromo-2-methylpropanoate via esterification and hydrosilylation. reacted with 2-bromoisobutyryl bromide (BIBB) and pyridine for $12 \mathrm{~h}$ to form the anchored initiator. ${ }^{255}$ Other initiators include 2bromopropionyl bromide which reacts with the oxide layer and triethylamine (TEA) at $0{ }^{\circ} \mathrm{C}$ during gentle agitation.

3.2.2 Sacrificial initiators. It is common that an additional free "sacrificial" initiator was added to form polymers in solution. Free initiators are added for two reasons, i.e. to help control polymerisations ${ }^{210,211,222,256,257}$ and allow other characterisation techniques as discussed below. ${ }^{242,258}$ A sacrificial initiator has a similar structure to a surface attached initiator, for example, Fig. 17A shows an ATRP initiator except it has no silane head group meaning that it will not form a monolayer and stay in solution. ${ }^{242,255,259}$ Polymers formed in solution can be analysed by gel permeation chromatography (GPC), or size exclusion chromatography ${ }^{\mathbf{1 2 4 , 2 4 6 , 2 5 5 , 2 6 0 , 2 6 1}}$ to calculate the degree of polymerisation. The polymers formed via free initiators are shown to correlate well with surface attached initiators. ${ }^{262,263}$ Average molecular weights, molecular weight spread and number average molecular weight can be determined from chromatograms, see examples in Fig. 17B and C, where (B) shows a very narrow polydispersity indicating a well-controlled polymerisation and (C) a wider polydispersity. Interestingly, due to the way that size exclusion chromatography works, larger polymers elute first which is unlike other types of chromatography, e.g. gas chromatography. As sacrificial initiators produce free polymer in solution, care must be taken to remove physisorbed free polymers for certain characterisation techniques of substrates, such as AFM.

3.2.3 Catalyst systems. The catalyst system is responsible for the creation of the radical by cleavage of the alkyl halide bond. As shown in Scheme 4, the equilibrium of the reaction is shifted to the left indicating that only a relatively small amount of chains have active species. This reduces the possibility of irreversible radical-radical termination. ${ }^{238,264}$ The persistent radical effect is thought to reduce $\mathrm{P}-\mathrm{P}$ termination as well as increasing the controllability whilst accelerating polymerisation. ${ }^{28,106,247,252}$ In addition to this, the Matyjaszewski and the Sheiko Groups showed that using additional quantities of the deactivator could improve the polymer brush by controlling the anticipated persistent radical effect. ${ }^{223,247,265}$ The radicals can reversibly deactivate back to dormant polymer chains, which also means that the metal is reduced to the lower oxidation state. Table 2 shows a selection of the catalyst systems used in rest of this review. A more in depth review of copper complexes can be found in ref. 266 .

3.2.4 ARGET and polymer brushes. Activators ReGenerated by Electron Transfer (ARGET) is a development of ATRP and can be used to reduce the concentration of metal catalysts up to 1000 times to ppm levels. ${ }^{241,249,257,270-272}$ In addition, polymerisations can be completed in limited amount of air so reactions do not have to be deoxygenated..$^{241,258,271}$ In ordinary ATRP, the effect of oxygen is that $\mathrm{Cu}(\mathrm{I})$ is oxidised without being halogenated as illustrated in Scheme 5, showing the transition back to $\mathrm{Cu}(\mathrm{I})$ due to the reducing agent. In ATRP, a small amount of oxygen can result in a large drop in the rate of polymerisation. ARGET ATRP overcomes this problem by having a readily available source of a reducing agent. Therefore 
A<smiles>CCOC(=O)C(C)(C)Br</smiles>
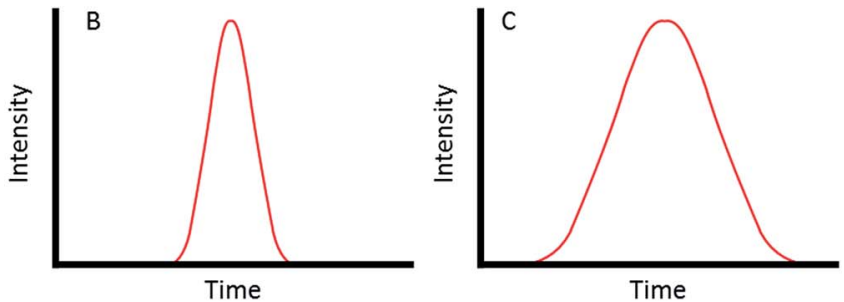

Fig. 17 (A) Is the structure of a frequently used sacrificial initiator, ethyl $\boldsymbol{\alpha}$-bromoisobutyrate. (B and $\mathrm{C}$ ) are examples of molecular weight distributions.

any $\mathrm{Cu}$ (II) generated is reduced back to the useful $\mathrm{Cu}(\mathrm{I})$. Zhu et al. used polydopamine as an ATRP initiator, methyl methacrylate as a monomer and (+)-sodium L-ascorbate as an ARGET. ${ }^{267}$ After 24 hours of polymerisation characterisation shows that $72 \mathrm{~nm}$ of methyl methacrylate brush was formed on top of the $58 \mathrm{~nm}$ initiator. In addition, over 72 hours $239 \mathrm{~nm}$ of methyl methacrylate was formed.

\subsection{Polymer brushes synthesis}

One of the main advantages of polymer brushes formed by ATRP is the controllability of the polymerisations. ${ }^{275}$ Similar to SAMs, polymer brushes are influenced by a range of factors such as temperature, solvent selection, presence of oxygen and free initiators. Unless otherwise mentioned, in this section polymer brushes are synthesised through selfassembling initiators and subsequent polymerisation from the active sites. An example is given in Scheme 6, showing a simplistic view of the polymerisation procedure from surface attached initiator monolayers to polymer growth. This was used by Munirasu et al. in forming polymer brushes of benzyl methacrylate with a copper catalyst, PMDETA ligand and free sacrificial initiator. ${ }^{259}$
3.3.1 Free initiators. It has been reported that the addition of deactivators or free initiators are needed to control polymerisations to create uniform polymer films. ${ }^{276}$ Öztürk et al. formed lauryl acrylate polymer brushes in the pursuit of an ultrahydrophobic surface. ${ }^{261}$ With the addition of ethyl- $\alpha$-bromoisobutirate (EBIB) as a free initiator the authors reported a linear trend between thickness and time. Characterized by AFM, it was found that when the brushes collapsed in some places as seen in Fig. 18. However, the relative roughness of the surface was found to be $0.8-1.6 \mathrm{~nm}$. They successfully achieved the maximum contact angle of $163^{\circ} \pm 2.8$. They also found that the water contact angle increased linearly with the immersion time and suggested that the thickness increased linearly with the time, hence concluded that the thickness was a tuneable parameter that could be accurately reproduced. ${ }^{261}$

Turan et al. studied the formation of $\operatorname{poly}(N$-isopropylacrylamide) with the addition of a chain transfer agent 2mercaptoethanol. Using varied amounts of 2-mercaptoethanol polymer brushes were formed at $90{ }^{\circ} \mathrm{C}$ over $18 \mathrm{~h}$. The concentration of 2-mercaptoethanol was shown to control the chain length of the polymer brush with smaller concentrations dictating longer chain lengths. However, higher concentrations showed lower roughness although with reduced chain
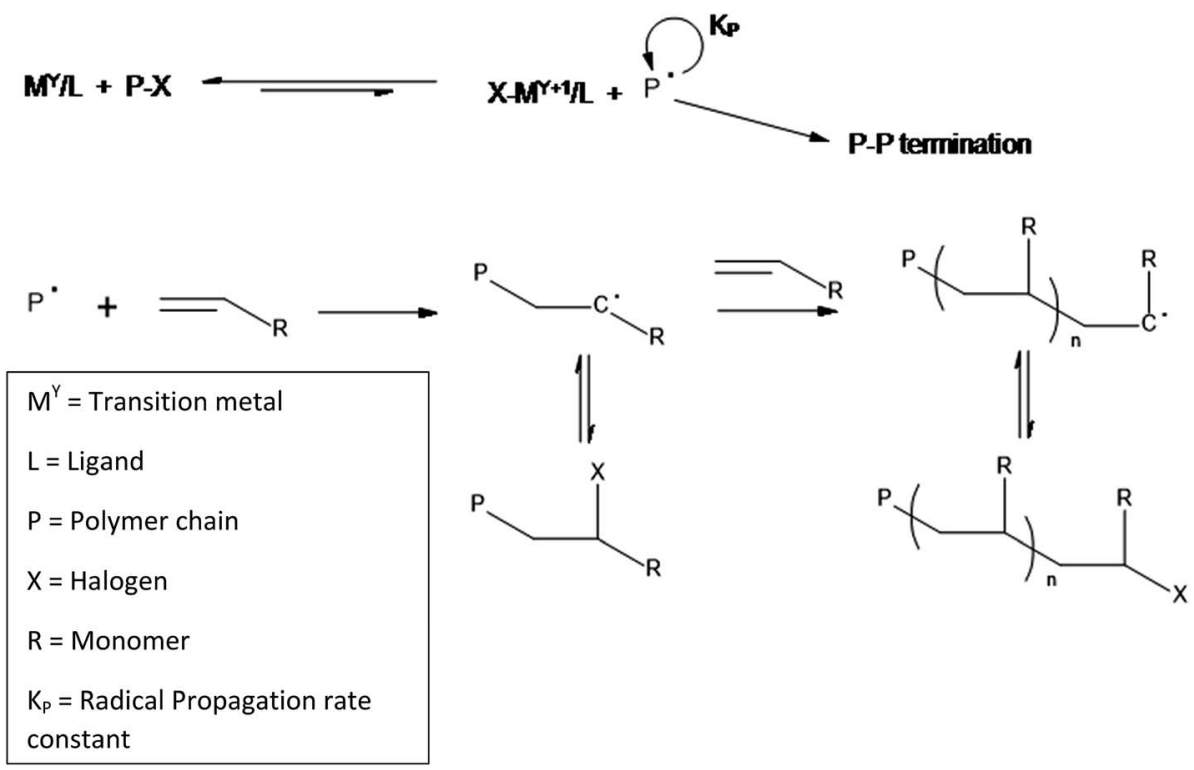

Scheme 4 Showing the mechanism of ATRP with metal complex. 
Table 2 A non exhaustive selection of catalyst systems in this review

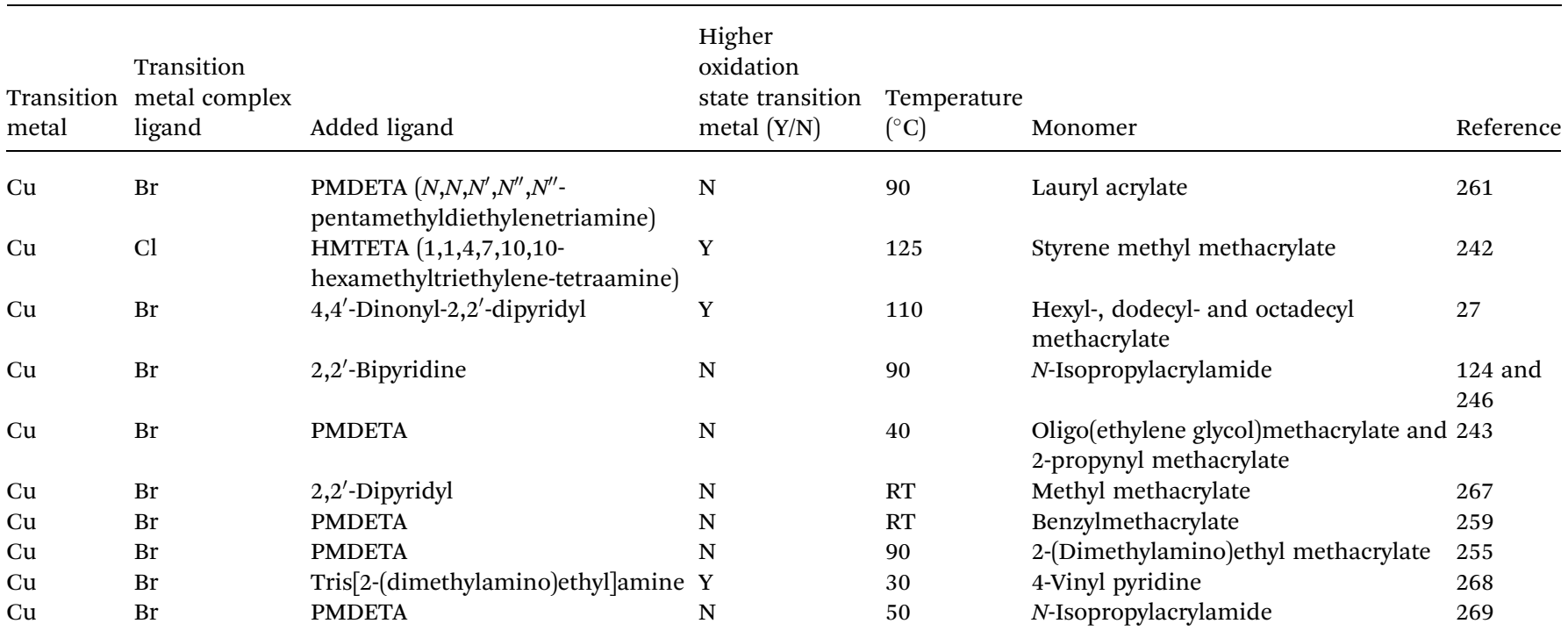

\section{Reducing Agent}

Such as ascorbic acid, sodium ascorbate, Tin (II) 2-ethylhexanoate, hydrazine, glucose or phenols

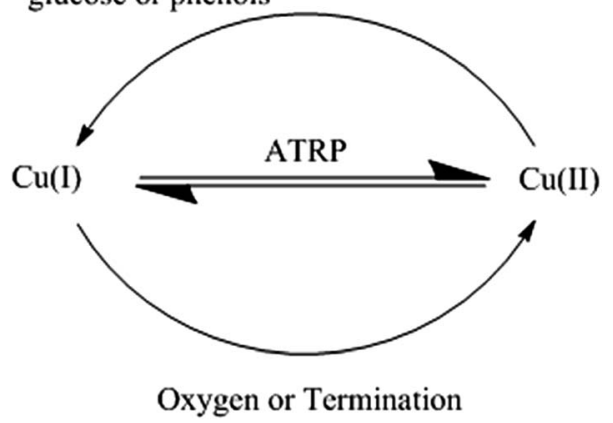

Scheme 5 Illustrating how the reducing agent can regenerate lost $\mathrm{Cu}(\mathrm{I})$ through oxidation or termination. Using information from ref. 211, $241,258,267,270,273$ and 274.

density. ${ }^{124}$ Turan et al. conducted another study on poly $(\mathrm{N}$-isopropylacrylamide) polymer chains although without using an extra chemical additive to control polymerisation. Ellipsometry measurements showed a dry thickness of $66 \mathrm{~nm}$. Munirasu et al. synthesised benzylmethacrylate polymer brushes as well as diblock copolymers with styrene on silicon wafer. ${ }^{259}$ Benzylmethacrylate polymer brushes followed a linear trend with time showing that brush length can be controlled with precision due to the additional EBIB. Their GPC results from the free polymer showed very low polydispersity indicating that the surface attached polymer grew at a uniform rate. Styrene performed similarly in respect to the EBIB-thickness trends. Liu and coworkers studied methyl methacrylate polymers, at $90{ }^{\circ} \mathrm{C}$ they varied the amount of a free sacrificial initiator. The thickest film produced had the lowest amount of EBIB added which is in agreement with Öztürk et al. Table 3 shows how the concentration of EBIB affects thickness, which is inherently correlated to $M_{\mathrm{n}}$ (number average molecular weight). Also notable is the slight decrease in polydispersity with minimal effect on contact angle.

The same experiments were done with solely styrene except at an elevated temperature by Liu et al. ${ }^{242}$ The data shows an irregular trend with $50 \mathrm{mM}$ showing twice the thickness of the next highest thickness. The authors also created a random copolymer, using a ratio of methyl methacrylate to styrene of $2: 1$ and a range of EBIB concentrations the best compromise for conversion of monomers and thickness of film was found to be $50 \mathrm{mM}$ however the thickest film was created by using 25 $\mathrm{mMol} \mathrm{L}^{-1}$. When the ratios of monomers was inverted, the thickness was less predictable much like what was shown when the styrene was the only monomer limiting the influence of EBIB as a controlling sacrificial initiator. Zhou et al. constructed poly(2-(dimethylamino)ethylmethacrylate) brushes on silica particles, where a native oxide layer was found similar to that on silicon wafers and other silicon based substrates. Free polymer that was produced through the addition of EBIB was precipitated, this allowed gel permeation chromatography to take place. GPC results allow the calculation of $M_{\mathrm{n}}$ and molecular weight average $\left(M_{\mathrm{w}}\right)$ in addition to increasing the control of the reaction. ${ }^{255}$

3.3.2 Polymerisation time and temperature. In addition to the effects of free initiators, polymerisation time and temperature can affect the final polymer that is formed. It is still worth considering that both temperature and time are inherently linked to a plethora of other variables. Temperature can have either positive or negative effects to polymerisations at elevated temperatures. For example, better control over the polymerisations may occur but this can lead to catalyst degradation or side reactions increasing. ${ }^{28}$ In addition, the increase in temperature positively influences the activation rate constants especially for less reactive initiators. ${ }^{277}$ 


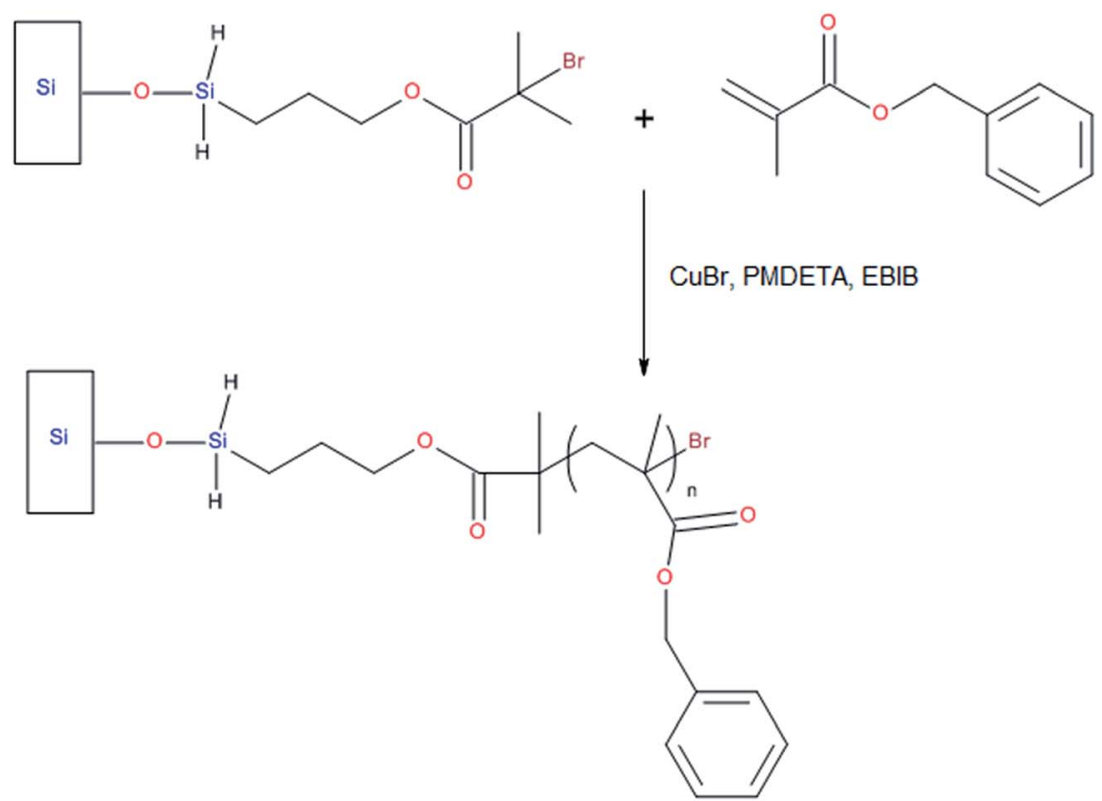

Scheme 6 A schematic of the simplified ATRP process.
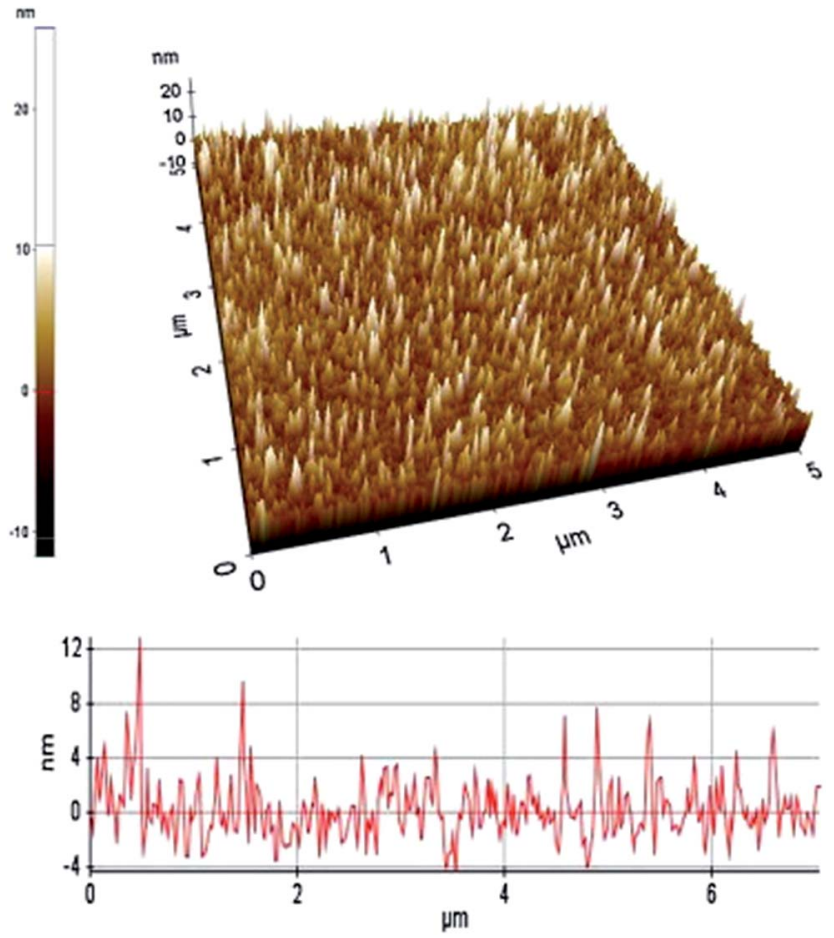

Fig. 18 Showing lauryl acrylate polymer brushes, some of which are partially collapsed and below a cross section. Reprinted with permission from ref. 261.

When considering polymerisation time it is worth noting that at high levels of monomer conversion the rate of propagation will slow dramatically and end group functionality may be lost. ${ }^{28}$ Munirasu et al. synthesised benzylmethacrylate polymer brushes and achieved a thickness of above $300 \mathrm{~nm}$ after polymerisation for 37 hours as seen in Fig. $19 .{ }^{259}$ Fig. 19 also clearly shows a linear trend with time. Diblock polymers were synthesised to show that the end groups are still intact therefore polymerisation was not complete and end group functionality still remained.

Liu and co-workers synthesized polymer brushes from methyl methacrylate, styrene and a random copolymer consisting of both. ${ }^{242}$ When the authors studied methyl methacrylate as a standalone monomer, the conversions reached $99 \%$ at $90{ }^{\circ} \mathrm{C}$ with a small amount of EBIB. However, for styrene on its own, the conversion changed from $10 \%$ at $90{ }^{\circ} \mathrm{C}$ to $95 \%$ at 125 ${ }^{\circ} \mathrm{C}$ as can be seen in Fig. 20. ${ }^{242}$

Song et al. produced a copolymer of oligo(ethylene glycol) methacrylate and 2-propynyl methacrylate. ${ }^{243}$ This was introduced in a $60: 40$ ratio to form the polymer brush. After $12 \mathrm{~h}$ at $40{ }^{\circ} \mathrm{C}$ the thickness of the brushes was found to be $26.8 \mathrm{~nm}$. Interestingly, after formation of these films they were used for click chemistry, specifically click glycosylation. ${ }^{243}$ Zhou et al. formed poly(2-(dimethylamino)ethylmethacrylate) brushes over $12 \mathrm{~h}$ at $90{ }^{\circ} \mathrm{C}$. Free polymer that was produced through the addition of free sacrificial initiator was precipitated, allowing gel permeation chromatography to take place and number average $\left(M_{\mathrm{n}}\right)$ was 6940 and molecular weight average $\left(M_{\mathrm{w}}\right)$ 11 870. Although polymerisation time was 4 hours less there is a considerable gap to the results produced by Liu et al. as can be seen in Table $3 .^{255}$

3.3.3 Summary. Much like SAMs, there are a large amount of variables to consider before embarking on polymer brush synthesis. With respect to surface attached initiators, the principles of SAMs must not be forgotten due to the essential monolayer. Also highlighted here is the role of free sacrificial initiators and their role in controlling the polymerisation. This is something that should be considered as the free polymer can then be analysed giving a detailed representation of the polymerisation success as well as the state of the attached polymer. 
Table 3 Results of ATRP of methyl methacrlate with EBIB as a free initiator ${ }^{a}$

\begin{tabular}{|c|c|c|c|c|c|}
\hline $\begin{array}{l}\text { Concentration of } \\
\text { EBIB }\left[\mathrm{mMol} \mathrm{L}^{-1}\right]\end{array}$ & Conversion of MMA & $M_{\mathrm{n}}\left[\mathrm{g} \mathrm{mol}^{-1}\right]$ & PDI & Thickness [nm] & Contact angle $\left[{ }^{\circ}\right]$ \\
\hline 20 & $77 \%$ & 46800 & 1.4 & 30.5 & 72 \\
\hline 60 & $93 \%$ & 28300 & 1.3 & 26.4 & 72 \\
\hline 80 & $99 \%$ & 22400 & 1.1 & 23.7 & 73 \\
\hline 100 & $99 \%$ & 17400 & 1.1 & 17.1 & 73 \\
\hline
\end{tabular}

${ }^{a}$ Reprinted with permission from ref. 242.

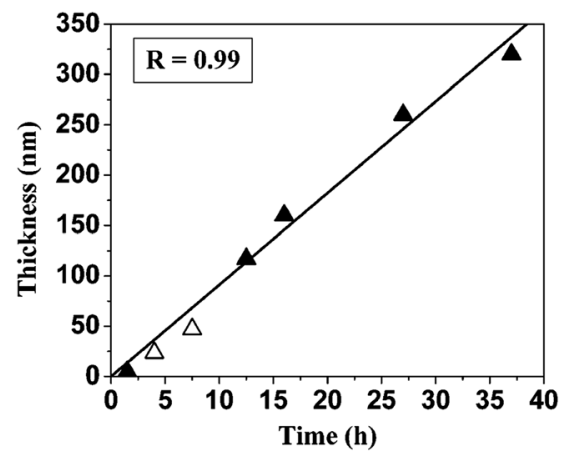

Fig. 19 Showing the linear relationship between time and thickness of benzylmethacrylate polymer brushes. Reprinted with permission from ref. 259.

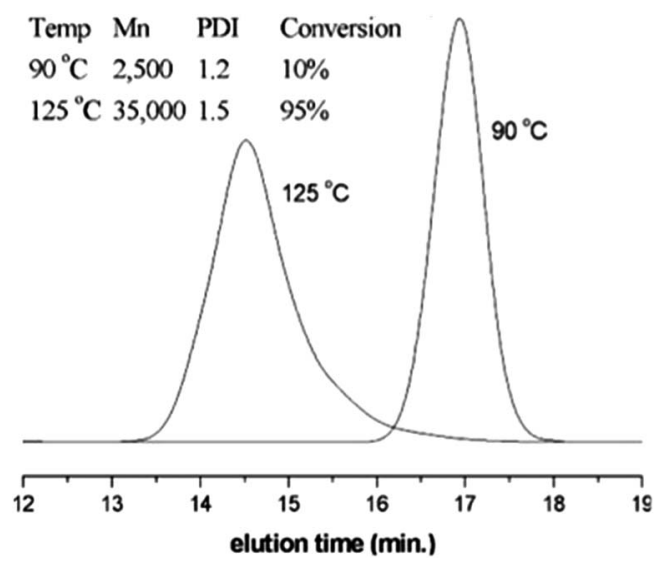

Fig. 20 GPC results indicating low conversion of styrene at lower temperatures with increased polydispersity. Reprinted with permission from ref. 242

The temperatures covered range from $40-90{ }^{\circ} \mathrm{C}$, although the lower temperature obtained a much thinner film it is possible to conduct polymerisation at lower temperatures. As previously stated excessive temperatures can result in catalyst quality reduction and an increase in side reactions. The catalyst complex must take into account the kinetics of polymerisation, included in this is also the unique monomeric atom transfer equilibrium constant. If the constant is too small polymerisation will be very slow. Solvent selection broadly has the same considerations as in SAM. In addition, the solubility of the catalyst must be considered although this is linked to ligand selection and temperature. Adequate control over polymer synthesis will produce the best films in terms of density and uniformity as well as reproducibility. Following this, there have been various developments of ATRP that facilitate the desirable end polymers, such as the previously mentioned ARGET and sacrificial initiators.

\subsection{Polymer brushes on silicon wafers for tribological applications}

The tribological properties of polymer brushes are dependent on the surface attachment, the swelling behaviour of brushes if a solvent is present, the type of stress encountered as well as contact pressures. ${ }^{121}$ The pioneers of polymer brushes for tribology, Klein et al., also realised the importance of a good solvent to facilitate swelling and therefore better sliding performance. ${ }^{278}$ The immersion of polymer brushes in good solvent allows brush swelling, known to help lubricate as illustrated in Fig. 21..$^{279-281}$ The mechanism in which solvated polymer brushes lubricate is thought to be as follows. ${ }^{121,282}$

(A) The resistance to rearrangement of the grafted chains with the repulsive nature of the brushes.

(B) Lubricant entrapment in the polymer brushes.

(C) High concentrations of lubricant in the outer polymer brush creates a low shear area protecting the brush system.

3.4.1 Brush thickness and COF. Thickness of polymer brushes inherently has an effect on the lubrication system $^{283}$ Kang et al. and Bielecki et al. amongst others have been able to correlate polymer brush growth to polymerisation time to confirm expected trends as can be seen in ref. 121 and 227 . Bielecki et al. synthesised methacrylates with different side chain lengths via SI-ATRP using hexyl-(P6MA), dodecyl-(P12MA) and octadecyl methacrylate (P18MA) monomers. ${ }^{27}$ Tribological studies were conducted using a ball on disk NTR2 tribometer in a reciprocating motion over $120^{\circ}$ with the speed change being controlled sinusoidally. Ellipsometry measurements of the dry thickness of the P6MA, P12MA and P18MA polymer brushes showed thicknesses of 90, 250 and $230 \mathrm{~nm}$ respectively (Fig. 22).

In a 20 cycle test with toluene all the brushes showed a low COF in the range of 0.01-0.02. It is worth noting that the much lower thickness in the P6MA polymer did not rule out a low COF in this case. The same experiment was completed using hexadecane, ethanol and PF350 oil as shown in Fig. 23. The improved reduction in friction when lubricated with non-polar 

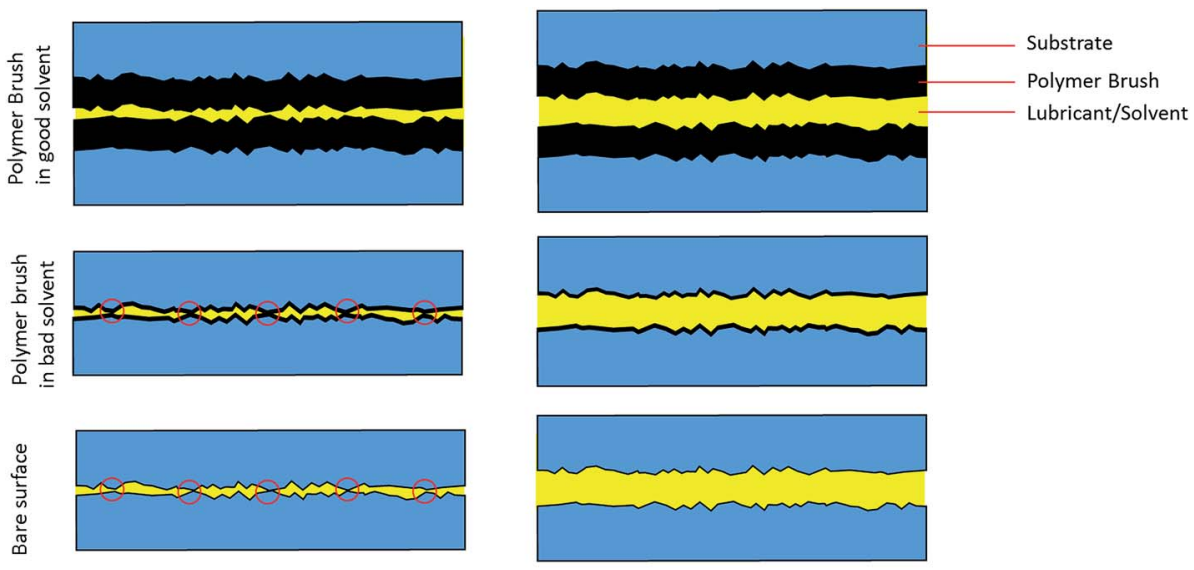

Fig. 21 An illustration of swollen polymer brushes lubricating a contact in comparison to poor solvent and a bare surface. Note the possible asperity contacts in poor solvent.

liquids may be due to brush swelling improving the lubricating qualities as mentioned above. In this experiment the two longer chain and thicker polymer films faired considerably better than P6MA which increased the COF of friction. In all cases the lubricating qualities of the PF350 were higher than that of hexadecane. When the authors selected P12MA and created three different thicknesses of polymer, 70, 140 and $250 \mathrm{~nm}$ thick, the two lower coatings lost their friction reducing characteristics within a few meters. The $250 \mathrm{~nm}$ polymer film maintained a COF of 0.012 over more than $100 \mathrm{~m}$.

The authors put this down to the fact that a thicker polymer film can be compressed more and reduce the likelihood of two hard counterparts coming into contact and the destructive high pressures destroying coatings. The application of a more viscous lubricant on the two thinner polymer coatings reduced the COF although the chemical instability may have been changed as there was a rapid failure of the $70 \mathrm{~nm}$ thick polymer in EO500 oil. The authors concluded that a greater initial brush

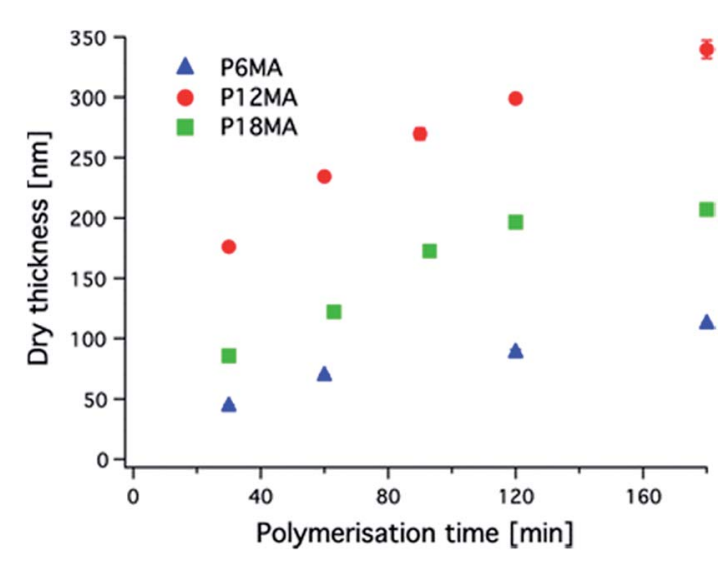

Fig. 22 Hexyl-, dodecyl- and octadecyl methacrylate polymer brushes formed from ATRP. Dry thicknesses measured by ellipsometry. Reprinted with permission from ref. 121. thickness was more effective in separating the surfaces. ${ }^{27}$ Bhairamadgi et al. compared the adhesion and frictional characteristics of a polymer brush that was synthesised using fluorinated monomers to non-fluorinated monomers. ${ }^{284}$ The fluorinated polymer brush showed a strong correlation between thickness and adhesive pull off force in the tested range of 5-40 N. At every loading test brushes between 65-140 nm, thicker brushes resulted in a lower pull off force, between 9-29 nm there was some discrepancy but within error bars. Non fluorinated polymer brushes proved too high to measure. Lateral friction loading showed good correlation with other studies on polymer brush length $v s$. COF. Fluorinated polymer brushes of between 10-140 nm were analysed and the COF was reduced in an almost linear fashion throughout to a lowest friction of 0.0057. Similar to adhesion testing fluorinated polymers proved to be more successful at reducing friction than their nonfluorinated counterparts. The length of polymer brushes is also investigated by Sakata et al. who found that shorter chains of methyl methacrylate had a much larger margin of error. ${ }^{281}$ The authors also concluded a good quality solvent allows the

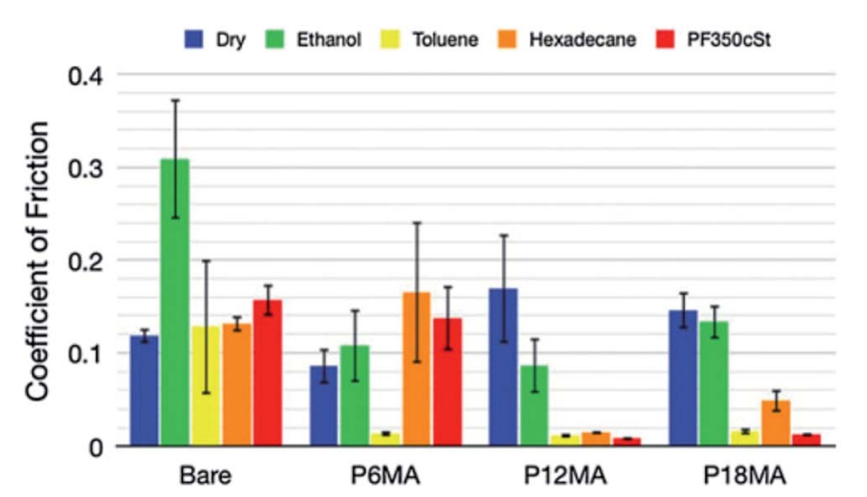

Fig. 23 COF values for the polymer brushes in different lubricants or dry conditions. Reprinted with permission from ref. 27. 
extension of the brushes and in poor solvent they can be seen to be compressed. This was confirmed by tribological testing in good solvents, toluene and acetone, and bad solvents, hexane and cyclohexane. Similar behaviour has been observed with poly(2-methacryloyloxyethylphosphorylcholine) brushes and the swelling effects in poly(methylmethacrylate). ${ }^{285,286}$ However, these brushes are hydrophilic so water can provide a suitable aqueous lubricant. Nomura et al. varied the composition of solvent to subsequently effect the swelling of a polystyrene brush and therefore the lubricating qualities of the modification. ${ }^{262}$ Although not created by ATRP, Limpoco et al. produced $117.6 \mathrm{~nm}$ polystyrene brushes. ${ }^{287}$ The authors then varied solvent and Fig. 24 shows the effect. This data was collected through AFM measurements and shows that brush swelling not only effects macrotribology.

Much like SAMs, friction is affected by the length of preformed adsorbed polymers. This is shown by Lin et al. who used the grafting-to approach. ${ }^{123}$ Three different copolymer brushes, poly(propylene oxide)-poly(ethylene oxide)-poly(propylene oxide), (PPO-PEO-PPO), were used as lubricants. Namely a PPO-PEO-PPOs with a molecular weights of 2700 with $60 \%$ PPO, 2150 with $80 \%$ PPO, 3100 with $80 \%$ PPO, designated as $17 \mathrm{R} 4,17 \mathrm{R} 2$ and $25 \mathrm{R} 2$ respectively. Tribological testing was carried out using a pin on disk tribometer. Ellipsometry measurements show that the thickest film produced was from the 25R4 polymer and reached around $6 \mathrm{~nm}$, the thinnest film was $17 \mathrm{R} 4$ which reached nearly $1 \mathrm{~nm}$. This again highlights the differences between grafting to and grafting from procedures. The better tribological performances were attributed to the longer chains and thicker films that had been formed.

3.4.2 Sliding speed and load vs. COF. Bielecki et al. constructed Stribeck curves for the ATRP synthesised polymer brush of P12MA monomers. ${ }^{27}$ Under a constant load the brushes showed a good correlation with the shape of a Stribeck curve when lubricated with hexadecane and a range of oils with viscosities from 36-1300 cSt. In addition, the brushes all showed an improvement in friction than the bare lubricated substrate. Bhairamadgi et al. synthesized fluorinated polymer brushes, as expected, in both AFM adhesion and lateral friction

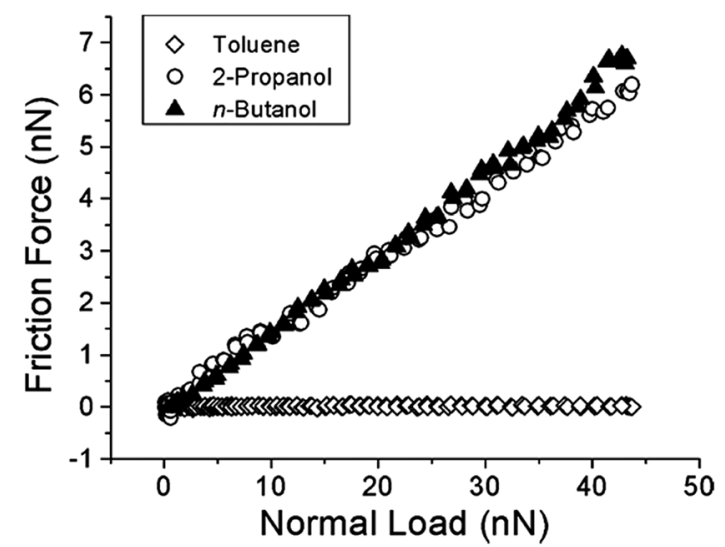

Fig. 24 AFM friction force vs. normal load in good and bad solvents. Reprinted with permission from ref. 287. testing the increase of load increased the pull off force and friction force. ${ }^{284}$ In a range of loads from 10-90 $\mathrm{nN}$ the friction force increased in a linear manner across all thicknesses. Liu et al. studied poly( $N$-isopropylacrylamide) brushes formed via ATRP. ${ }^{269}$ After ATRP the samples were tested using a ball on disk tribometer using deionised water as a lubricant. Ellipsometry measurements gave a dry thickness of the brush to be approximately $76.2 \mathrm{~nm}$. The grafted brush achieved the lowest friction which stabilised at 0.03 for $1062 \mathrm{~s}$ when it was reduced to 0.01 under a load of $0.78 \mathrm{~N}$ and sliding speed of $41.66 \mathrm{~mm} \mathrm{~s}^{-1}$. In the same conditions the polymer lubricant reduced the COF to 0.1, at a higher sliding speed of $55.54 \mathrm{~mm} \mathrm{~s}^{-1}$ the grafted polymer did not change significantly whilst the lubricant approached a COF of 0.125 . With an increased load of $0.98 \mathrm{~N}$ the grafted reduced the COF less and at a higher sliding speed this was reduced once again. The polymer lubricant followed the same trend. The authors presented Fig. 25 showing how the polymer brushes may interact with the deionised water as a lubricant with and without compressive forces. Heeb et al. also used water as a base for a buffer solution to lubricate hydrophilic brushes. ${ }^{\mathbf{2 8 0}}$ Tribological testing of poly(methacrylic)acid brushes under similar but slower conditions (pin on disk, $1 \mathrm{~N}$, sliding speeds of $0.25-10 \mathrm{~mm} \mathrm{~s}^{-1}$ ) and achieved COF under 0.005 for each test.

The polymer brushes that Lin et al. previously introduced consistently reduce friction over the range of $0.01-0.1 \mathrm{~m} \mathrm{~s}^{-1} .{ }^{123}$ The effect of applied load was investigated by changing the load from 4-8 $\mathrm{N}$ under a constant sliding speed of $0.01 \mathrm{~m} \mathrm{~s}^{-1}$ and constant concentration. The notable changes in the COF are from 17R4 which increased between 4 and $5 \mathrm{~N}$ then levelled off,

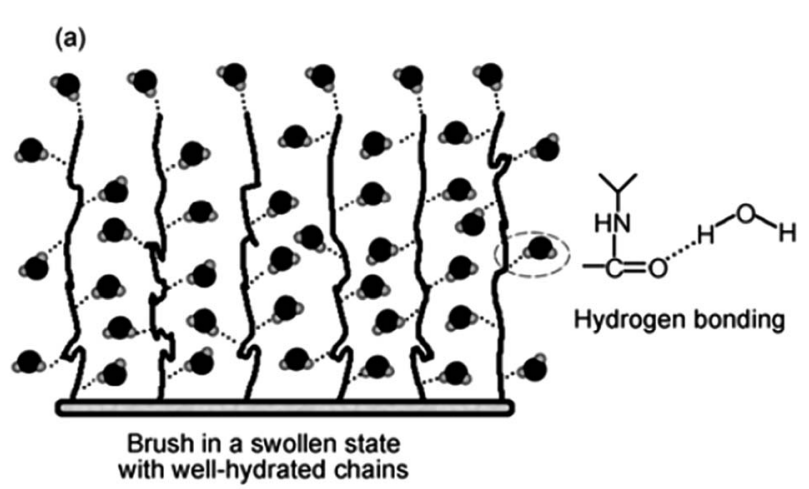

(b)

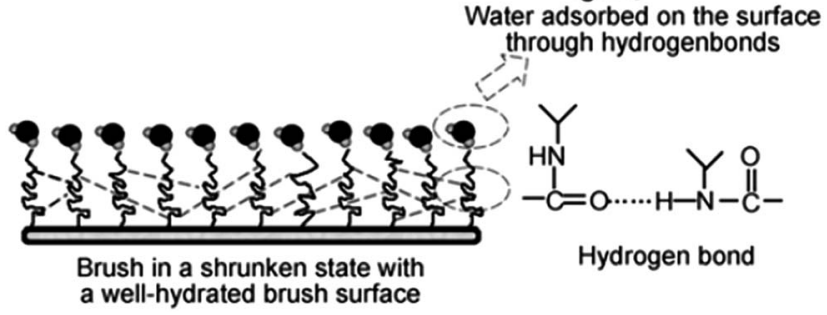

Fig. 25 A representation of how polymer brushes can react with and without the presence of load. Reprinted with permission from ref. 269. 
25R2 shows a slight increase in COF throughout the entire test but until approximately $7.5 \mathrm{~N}$ surpassed the other polymers in friction reduction. 17R2 consistently showed COF values below 0.15 from $4-7 \mathrm{~N}$ where it sharply reduced. The COF fell below that of $15 \mathrm{R} 2$ after $7.5 \mathrm{~N}$. Sun et al. sought to improve the tribological resistance of a polyamic acid polyimide film (PI). ${ }^{276}$ They achieved this by synthesizing polyglycidyl methacrylate brushes that are epoxy ended as an adhesive layer before application of the PI film. This both increased the lifetime of the film from 6000 sliding cycles to in excess of 25000 under a $0.5 \mathrm{~N}$ load at $20 \mathrm{~mm} \mathrm{~s}^{-1}$ with a consistently low COF of 0.08 .

3.4.3 Summary. As previously discussed there are numerous variables to be considered before embarking on polymer brush synthesis. However, polymer brushes have proven that low COF is possible. For NEMS and MEMS devices it should be noted that in an absence of liquid lubricant there will be no brush swelling, however, fluorinated polymer brushes have shown to reduce adhesion and pull off force creating opportunities for lubrication solutions in this field. In liquid lubricated systems it has been shown repeatedly that swollen brushes have better tribological properties in good solvent, for future applications this is key. With thicker swollen brushes a key issue, synthesizing thicker polymer brushes, has shown to resist the effects of load by coping with the compressive forces better than thinner polymer films. The thicker brushes have proven to reduce contact between surface asperities and therefore reduce friction as well as the shearing of the polymer. Polymer brushes could be used to reduce wear, however as soon as the polymer brushes are removed wear will occur. The attachment of the polymer brush to the silicon wafer is key to this.

\subsection{Polymer brushes on silicon nitride}

Polymer brushes have been formed by ATRP on surfaces from silicon nitride cantilevers to wafers. However, very few tests have been done regarding tribology. de Groot et al. used a silicon nitride nanoporous substrate to form poly(methacrylic acid) brushes using ATRP. ${ }^{288}$ The ATRP was deposited via vapour phase deposition over $16 \mathrm{~h}$. Sodium methacrylate was polymerised and in $1 \mathrm{~h}$ ellipsometry show that the film thickness was around $60 \mathrm{~nm}$ thick. The swell and collapse of the polymer brushes were also investigated using AFM. Nguyen et al. constructed a zwitterionic polymer brush on silicon nitride using ATRP. ${ }^{226}$ The silicon nitride was deposited upon silicon wafers using low pressure chemical vapour deposition, after etching with HF, 1,2-epoxy-9-decene was attached with UV light. After immersion in 1,2-ethlyamine the ATRP bromoisobutyryl bromide was attached using aforementioned techniques. The polymer brush of [3-(methacryloylamino)propyl]dimethyl(3sulfopropyl)ammonium hydroxide inner salt monomers formed over $20 \mathrm{~nm}$ of growth in the first hour and $70 \mathrm{~nm}$ in $8 \mathrm{~h}$. A later paper by Nguyen and co-workers detailed the same procedure for attachment of initiator and polymerisation upon a silicon nitride surface. ${ }^{289}$ The purpose of both polymer brushes created by Nguyen was protein repulsion. Gabriel et al. have created brushes on silicon nitride AFM probes, using electro initiated polymerisation techniques the authors successfully formed poly- $N$-succinimidylacrylate brushes. ${ }^{290}$ This was confirmed by approach and retraction curves using the cantilevers and a bare silicon substrate. The force curves could then be used as a form of sensing, much like monomers were used in CFM. Although not created by ATRP, Hartung et al. used a preformed copolymer brush like additive to lubricate $\mathrm{Si}_{3} \mathrm{~N}_{4}$ contacts. ${ }^{180}$ Poly(L-lysine)-graft-poly(ethylene glycol) (PLL- $g$-PEG) was dissolved in a buffer solution of 4-(2-hydroxyethyl) piperazine-1-ethanesulfonic acid in water (adjusted to $\mathrm{pH} 7$ ), and samples were immersed for 30 minutes before testing was carried out. The cationic backbone adsorbs onto a negatively charged surface, hence the need for a buffer. Ellipsometry showed that the dry film thickness was approximately $15.7 \mathrm{~nm}$. Tribological testing was completed using a pin on disk tribometer with a silicon nitride pin. Under a load of $5 \mathrm{~N}$ and speed of $120 \mathrm{~mm} \mathrm{~s}^{-1}$ the COF was 0.02 after running in under the buffer solution, after the addition of the buffer and polymer solution the COF dropped to 0.003 . The solution also effectively lubricated under a slower speed of $10 \mathrm{~mm} \mathrm{~s}^{-1}$ under a load of 2 $\mathrm{N}$ which resulted in a COF of 0.04 . However, the polymer inhibits the tribochemical reactions leading to a roughened surface and micro fractures.

\subsection{Challenges of polymer brushes for lubrication}

As with SAM there is no standardised testing regime, subsequently this results in difficulties in comparing all polymer brushes. Previous studies show the importance of controlling polymer brushes, thicknesses, swelling behaviour, initiator attachment and catalyst systems, all of which and more must be considered when attempting polymer brush synthesis. As covered above the technology and ability to produce high quality polymer brushes is in existence. However, this will prove to be much more difficult when considering in situ lubrication, such as an additive like component in a lubricating fluid. This will be a main concern in macro systems, micro systems may well have similar issues. Another problem with in situ lubrication is the time required to produce the thicker brushes that have been shown to be capable of producing low COF. The stability of the $\mathrm{Cu}$ complex catalyst and the effect of the oxidative effects have been thoroughly documented, even with the developments of ARGET this will appear as a point of interest for the application of polymer brushes in years to come. It has also been found that the unstable copper catalyst could be reduced by iron and therefore lose its reactivity. ${ }^{27,291}$ Although this could be reversed by ARGET it becomes another complication of the polymer brush system. Although it has been shown that ATRP can be completed from silicon nitride and SAM attachment to silicon nitride has been proved, the lubrication of silicon nitride from SI-ATRP has not been completed yet.

\section{Outlook}

This review has summarised the basics and the key development in SAMs and polymer brushes with a focus on tribology. Several decades have passed since the original study of self- 
assembling thiols on gold surfaces and SAMs have shown their feasibility for NEMS and MEMS lubrication. Although research has thrived in MEMS/NEMS, research has also been conducted to develop SAMs in macro applications especially on the controlling of interfaces and wettability that are key to the success of using SAMs. A multitude of SAMs have shown significant friction reducing characteristics which opens up possibilities of additive applications in automotive engines, certainly with ease of application in comparison to polymer brushes, however the shearing of such monolayers is still a concern. This growing area of research will continue to thrive due to the diverse disciplines that provide limitless potential applications.

Following extensive research it is now possible to produce surface initiated polymer brushes upon surfaces with controlled polymerisations techniques. These living radical techniques such as ATRP have led to unrivalled control over chain length, architecture and composition. Polymer brushes are respected and established in polymer science and the interesting structures will lead to commercial opportunities. Limited research has been completed on the application of polymer brushes to silicon nitride especially with respect to lubrication. However, the ability to create thin polymer films with specific functions means that this challenge should be feasible. In addition, due to the structure of silicon nitride, lessons learned from silicon wafers and the expanding nanoparticle field should provide a bridging step. That said, the air sensitive nature of ATRP will be difficult to overcome to produce in situ polymerisations although developments such as ARGET could combat these problems. It should be remembered that polymer brushes for tribological applications encompasses a wide range of disciplines and all of the skills from these areas will be required to create the commercially viable solutions.

In summary, the authors think that the synthesis of polymer brushes through ATRP will meet the requirements of many tribological contacts. The wide range of monomers that can be polymerised, the robustness of the technique, coupled with developments such as ARGET are an attractive solution. Although we see polymer brushes as a solution for tribological contacts the very nature of surface initiation means the lessons learnt in SAMs must not be forgotten.

\section{Acknowledgements}

The authors acknowledge the financial support from the Defence Science and Technology Laboratory (DSTL, DSTLX1000093632), which is part of the UK's Ministry of Defence and from EPSRC (Grant EP/M50662X/1) to this project. The authors also thank D. Gropper for proofreading the manuscript.

\section{References}

1 K. Holmberg, P. Andersson and A. Erdemir, Tribol. Int., 2012, 47, 221-234.

2 K. Holmberg, P. Andersson, N.-O. Nylund, K. Mäkelä and A. Erdemir, Tribol. Int., 2014, 78, 94-114.
3 Z. Tang and S. Li, Curr. Opin. Solid State Mater. Sci., 2014, 18, 119-139.

4 M. Ratoi, V. B. Niste, H. Alghawel, Y. F. Suen and K. Nelson, RSC Adv., 2014, 4, 4278-4285.

5 M. Ratoi, V. Anghel, C. Bovington and H. A. Spikes, Tribol. Int., 2000, 33, 241-247.

6 S. Loehlé, C. Matta, C. Minfray, T. L. Mogne, R. Iovine, Y. Obara, A. Miyamoto and J. M. Martin, Tribol. Int., 2015, 82(Part A), 218-227.

7 V. Anghel, C. Bovington and H. A. Spikes, Lubr. Sci., 1999, 11, 313-335.

8 C. J. Reeves, P. L. Menezes, T.-C. Jen and M. R. Lovell, Tribol. Int., 2015, 90, 123-134.

9 H. Zhao, A. Neville, A. Morina, R. Vickerman and J. Durham, Tribol. Int., 2012, 46, 62-72.

10 T. Reddyhoff, I. S. Y. Ku, A. S. Holmes and H. A. Spikes, Tribol. Lett., 2011, 41, 239-246.

11 K. Topolovec Miklozic, J. Graham and H. Spikes, Tribol. Lett., 2001, 11, 71-81.

12 A. Morina, A. Neville, M. Priest and J. H. Green, Tribol. Int., 2006, 39, 1545-1557.

13 S. Jiang, R. Frazier, E. S. Yamaguchi, M. Blanco, S. Dasgupta, Y. Zhou, T. Cagin, Y. Tang and W. A. Goddard, J. Phys. Chem. B, 1997, 101, 7702-7709.

14 W. Yue, C. Liu, Z. Fu, C. Wang, H. Huang and J. Liu, Mater. Des., 2014, 64, 601-607.

15 M. Skjoedt, R. Butts, D. N. Assanis and S. V. Bohac, Tribol. Int., 2008, 41, 556-563.

16 J. A. Williams, Tribol. Lett., 2004, 17, 765-778.

17 L. Wang, M. Nie and J. Rumbol, Tribol.-Mater., Surf. Interfaces, 2012, 6, 75-83.

18 S. Korcek, J. Sorab, M. D. Johnson and R. K. Jensen, Ind. Lubr. Tribol., 2000, 52, 209-220.

19 H. Spikes, Lubr. Sci., 2008, 20, 103-136.

20 C. Haensch, S. Hoeppener and U. S. Schubert, Chem. Soc. Rev., 2010, 39, 2323-2334.

21 C. Vericat, M. E. Vela, G. Benitez, P. Carro and R. C. Salvarezza, Chem. Soc. Rev., 2010, 39, 1805-1834.

22 J. A. Williams and H. R. Le, J. Phys. D: Appl. Phys., 2006, 39, R201.

23 B. W. Ewers and J. D. Batteas, RSC Adv., 2014, 4, 1680316812.

24 R. L. Jones, B. L. Harrod and J. D. Batteas, Langmuir, 2010, 26, 16355-16361.

25 J. E. Raynor, J. R. Capadona, D. M. Collard, T. A. Petrie and A. J. García, Biointerphases, 2009, 4, FA3-FA16.

26 I. Luzinov, D. Julthongpiput, V. Gorbunov and V. V. Tsukruk, Tribol. Int., 2001, 34, 327-333.

27 R. Bielecki, M. Crobu and N. Spencer, Tribol. Lett., 2013, 49, 263-272.

28 K. Matyjaszewski and J. Xia, Chem. Rev., 2001, 101, 29212990.

29 A. Ulman, Chem. Rev., 1996, 96, 1533-1554.

30 R. G. Nuzzo and D. L. Allara, J. Am. Chem. Soc., 1983, 105, 4481-4483.

31 S. A. Kulkarni, S. A. Mirji, A. B. Mandale, R. P. Gupta and K. P. Vijayamohanan, Mater. Lett., 2005, 59, 3890-3895. 
32 T. Manifar, A. Rezaee, M. Sheikhzadeh and S. Mittler, Appl. Surf. Sci., 2008, 254, 4611-4619.

33 F. Schreiber, Prog. Surf. Sci., 2000, 65, 151-257.

34 S. Frank, J. Phys.: Condens. Matter, 2004, 16, R881.

35 K. Bierbaum, M. Grunze, A. A. Baski, L. F. Chi, W. Schrepp and H. Fuchs, Langmuir, 1995, 11, 2143-2150.

36 D. K. Aswal, S. Lenfant, D. Guerin, J. V. Yakhmi and D. Vuillaume, Anal. Chim. Acta, 2006, 568, 84-108.

37 T. Balgar, R. Bautista, N. Hartmann and E. Hasselbrink, Surf. Sci., 2003, 532-535, 963-969.

38 R. Walsh, Acc. Chem. Res., 1981, 14, 246-252.

39 J. Dong, A. Wang, K. Y. S. Ng and G. Mao, Thin Solid Films, 2006, 515, 2116-2122.

40 K. Bierbaum, M. Kinzler, C. Woell, M. Grunze, G. Haehner, S. Heid and F. Effenberger, Langmuir, 1995, 11, 512-518.

41 T. A. Witten and L. M. Sander, Phys. Rev. Lett., 1981, 47, 1400-1403.

42 M. M. Sung, C. Carraro, O. W. Yauw, Y. Kim and R. Maboudian, J. Phys. Chem. B, 2000, 104, 1556-1559.

43 I. Doudevski, W. A. Hayes, J. T. Woodward and D. K. Schwartz, Colloids Surf., A, 2000, 174, 233-243.

44 D. Appelhans, D. Ferse, H. J. P. Adler, W. Plieth, A. Fikus, K. Grundke, F. J. Schmitt, T. Bayer and B. Adolphi, Colloids Surf., A, 2000, 161, 203-212.

45 D. K. Schwartz, S. Steinberg, J. Israelachvili and J. A. N. Zasadzinski, Phys. Rev. Lett., 1992, 69, 3354-3357.

46 Y. Wang and M. Lieberman, Langmuir, 2003, 19, 1159-1167. 47 M. Salmeron, Tribol. Lett., 2001, 10, 69-79.

48 I. Choi, Y. Kim, S. K. Kang, J. Lee and J. Yi, Langmuir, 2006, 22, 4885-4889.

49 I. Doudevski and D. K. Schwartz, J. Am. Chem. Soc., 2001, 123, 6867-6872.

50 V. V. Tsukruk, I. Luzinov and D. Julthongpiput, Langmuir, 1999, 15, 3029-3032.

51 J. J. Benítez, S. Kopta, D. F. Ogletree and M. Salmeron, Langmuir, 2002, 18, 6096-6100.

52 Z. Kang, Q. Liu and Y. Liu, Wear, 2013, 303, 297-301.

53 N. Rozlosnik, M. C. Gerstenberg and N. B. Larsen, Langmuir, 2003, 19, 1182-1188.

54 D. K. Schwartz, Annu. Rev. Phys. Chem., 2001, 52, 107-137. 55 B. Cui, J. Zhang and J. Chen, Tribol. Int., 2013, 62, 149-154.

56 M. Masuko, H. Miyamoto and A. Suzuki, Tribol. Int., 2007, 40, 1587-1596.

57 N. Satyanarayana and K. S. Sujeet, J. Phys. D: Appl. Phys., 2005, 38, 3512.

58 S. Song, L. Liu and J. Zhang, Appl. Surf. Sci., 2011, 257, 10254-10260.

59 K. Wang, D. Xiong and Y. Niu, Appl. Surf. Sci., 2014, 317, 875-883.

60 D. Xiong, Y. Deng, N. Wang and Y. Yang, Appl. Surf. Sci., 2014, 298, 56-61.

61 L. G. Yu, E. S. Yamaguchi, M. Kasrai and G. M. Bancroft, Tribol. Int., 2011, 44, 692-701.

62 J. Zhao, M. Chen, Y. An, J. Liu and F. Yan, Appl. Surf. Sci., 2008, 255, 2295-2302.

63 B. D. Booth, N. J. Martin, E. A. Buehler, C. McCabe and G. K. Jennings, Colloids Surf., A, 2012, 412, 57-63.
64 K. Busuttil, N. Nikogeorgos, Z. Zhang, M. Geoghegan, C. A. Hunter and G. J. Leggett, Faraday Discuss., 2012, 156, 325-341.

65 J. Feng, G. H. Xu, Y. An and X. Zeng, Colloids Surf., A, 2008, 316, 194-201.

66 N. R. Glass, R. Tjeung, P. Chan, L. Y. Yeo and J. R. Friend, Biomicrofluidics, 2011, 5, 36501-36507.

67 D. S. Grierson and R. W. Carpick, Nano Today, 2007, 2, 1221.

68 J. Li and X. Z. Li, Appl. Surf. Sci., 2009, 255, 6159-6163.

69 S. Liu, J. Ou, Z. Li, S. Yang and J. Wang, Appl. Surf. Sci., 2012, 258, 2231-2236.

70 J. Q. Ma, C. J. Pang, Y. F. Mo and M. W. Bai, Wear, 2007, 263, 1000-1007.

71 M.-K. Park, G. Sakellariou, S. Pispas, N. Hadjichristidis and R. Advincula, Colloids Surf., A, 2008, 326, 115-121.

72 S.-L. Ren, S.-R. Yang, J.-Q. Wang, W.-M. Liu and Y.-P. Zhao, Chem. Mater., 2004, 16, 428-434.

73 C. Zhang, S. Zhang, P. Gao, H. Ma and Q. Wei, Thin Solid Films, 2014, 570(Part A), 27-32.

74 G. Yang, C. Zhang, S. Zhang, L. Yu and P. Zhang, Mater. Res. Bull., 2014, 55, 88-94.

75 N. Satyanarayana, S. K. Sinha and M. P. Srinivasan, in Tribology and Interface Engineering Series, ed. M. P. G. D. D. Dowson and A. A. Lubrecht, Elsevier, 2005, vol. 48, pp. 821-826.

76 S. Han, K. Suga, M. Fujihira and B.-E. Park, J. Korean Phys. Soc., 2014, 64, 63-68.

77 K. Raiber, A. Terfort, C. Benndorf, N. Krings and H.-H. Strehblow, Surf. Sci., 2005, 595, 56-63.

78 D. Skácelová, V. Danilov, J. Schäfer, A. Quade, P. Sťahel, M. Černák and J. Meichsner, J. Mater. Sci. Eng. B, 2013, 178, 651-655.

79 D. Skácelová, M. Stupavská, P. Sťahel and M. Černák, Appl. Surf. Sci., 2014, 312, 203-207.

80 L. Wu, L. Cai, A. Liu, W. Wang, Y. Yuan and Z. Li, Appl. Surf. Sci., 2015, 349, 683-694.

81 M. Wiegand, M. Reiche and U. Gösele, J. Electrochem. Soc., 2000, 147, 2734-2740.

82 V. Bhatt and S. Chandra, J. Electron. Mater., 2009, 38, 19791989.

83 R. Maboudian and C. Carraro, Annu. Rev. Phys. Chem., 2004, 55, 35-54.

84 D. Xu, W. H. Yu, E. T. Kang and K. G. Neoh, J. Colloid Interface Sci., 2004, 279, 78-87.

85 H. Sano, M. Zhao, D. Kasahara, K. Murase, T. Ichii and H. Sugimura, J. Colloid Interface Sci., 2011, 361, 259-269.

86 R. Boukherroub, Curr. Opin. Solid State Mater. Sci., 2005, 9, 66-72.

87 S. Y. Lee, Y. Choi, E. Ito, M. Hara, H. Lee and J. Noh, Phys. Chem. Chem. Phys., 2013, 15, 3609-3617.

88 S. Hsieh, W.-J. Chao, P.-Y. Lin and C.-W. Hsieh, Corros. Sci., 2014, 80, 427-433.

89 M. E. McGovern, K. M. R. Kallury and M. Thompson, Langmuir, 1994, 10, 3607-3614.

90 L. T. Zhuravlev, Langmuir, 1987, 3, 316-318. 
91 J. C. Love, L. A. Estroff, J. K. Kriebel, R. G. Nuzzo and G. M. Whitesides, Chem. Rev., 2005, 105, 1103-1170.

92 A. Borruto, G. Crivellone and F. Marani, Wear, 1998, 222, 57-65.

93 B. D. Beake, J. S. G. Ling and G. J. Leggett, J. Mater. Chem., 1998, 8, 2845-2854.

94 D. Devaprakasam, O. P. Khatri, N. Shankar and S. K. Biswas, Tribol. Int., 2005, 38, 1022-1034.

95 Z. Pawlak, W. Urbaniak and A. Oloyede, Wear, 2011, 271, 1745-1749.

96 R. Prioli, L. G. Jacobsohn, M. E. H. Maia da Costa and F. L. Freire Jr, Tribol. Lett., 2003, 15, 177-180.

97 Y. C. Jung and B. Bhushan, Langmuir, 2009, 25, 1416514173.

98 E. Bormashenko, Y. Bormashenko, G. Whyman, R. Pogreb, A. Musin, R. Jager and Z. Barkay, Langmuir, 2008, 24, 40204025.

99 K.-Y. Yeh, L.-J. Chen and J.-Y. Chang, Langmuir, 2008, 24, 245-251.

100 M. Callewaert, C. Grandfils, L. Boulangé-Petermann and P. G. Rouxhet, J. Colloid Interface Sci., 2004, 276, 299-305.

101 D. Song, B. Song, H. Hu, X. Du and Z. Ma, Appl. Therm. Eng., 2015, 85, 356-364.

102 S. Shibuichi, T. Yamamoto, T. Onda and K. Tsujii, J. Colloid Interface Sci., 1998, 208, 287-294.

103 D. Janssen, R. de Palma, S. Verlaak, P. Heremans and W. Dehaen, Thin Solid Films, 2006, 515, 1433-1438.

104 A. Cricenti, G. Longo, M. Luce, R. Generosi, P. Perfetti, D. Vobornik, G. Margaritondo, P. Thielen, J. S. Sanghera, I. D. Aggarwal, J. K. Miller, N. H. Tolk, D. W. Piston, F. Cattaruzza, A. Flamini, T. Prosperi and A. Mezzi, Surf. Sci., 2003, 544, 51-57.

$105 \mathrm{H}$. Cheng and Y. Hu, Adv. Colloid Interface Sci., 2012, 171172, 53-65.

106 H. Fischer, Macromolecules, 1997, 30, 5666-5672.

107 A. Checco, H. Schollmeyer, J. Daillant, P. Guenoun and R. Boukherroub, Langmuir, 2006, 22, 116-126.

108 J. Ma, J. Liu, Y. Mo and M. Bai, Colloids Surf., A, 2007, 301, 481-489.

109 B. Lego, M. François, W. G. Skene and S. Giasson, Langmuir, 2009, 25, 5313-5321.

110 C. Sun, M. Zhang, F. Zhou, P. Gao, Y. Xia and W. Liu, J. Macromol. Sci., Part B: Phys., 2011, 50, 1006-1017.

111 L. B. Picraux, C. D. Zangmeister and J. D. Batteas, Langmuir, 2006, 22, 174-180.

112 J. Tersoff and D. R. Hamann, Phys. Rev. Lett., 1983, 50, 1998-2001.

113 G. Binnig, H. Rohrer, C. Gerber and E. Weibel, Phys. Rev. Lett., 1982, 49, 57-61.

114 G. Binnig and H. Rohrer, Rev. Mod. Phys., 1987, 59, 615625.

115 M. Sprik, E. Delamarche, B. Michel, U. Roethlisberger, M. L. Klein, H. Wolf and H. Ringsdorf, Langmuir, 1994, 10, 4116-4130.

116 K. Edinger, A. Goelzhaeuser, K. Demota, C. Woell and M. Grunze, Langmuir, 1993, 9, 4-8.
117 L. Qin, W. Zhao, H. Hou, Y. Jin, Z. Zeng, X. Wu and Q. Xue, RSC Adv., 2014, 4, 60307-60315.

118 S. V. Merzlikin, N. N. Tolkachev, T. Strunskus, G. Witte, T. Glogowski, C. Wöll and W. Grünert, Surf. Sci., 2008, 602, 755-767.

119 W. A. M. Aarnink, A. Weishaupt and A. van Silfhout, Appl. Surf. Sci., 1990, 45, 37-48.

120 A.-S. Duwez, J. Electron Spectrosc. Relat. Phenom., 2004, 134, 97-138.

121 R. Bielecki, E. Benetti, D. Kumar and N. Spencer, Tribol. Lett., 2012, 45, 477-487.

122 M. Biesalski and J. Rühe, Macromolecules, 2003, 36, 12221227.

123 B. Lin, A. K. Tieu, H. Zhu, B. Kosasih, O. Novareza and G. Triani, Wear, 2013, 302, 1010-1016.

124 E. Turan and T. Caykara,J. Polym. Sci., Part A: Polym. Chem., 2010, 48, 3880-3887.

125 J. J. Gooding, F. Mearns, W. Yang and J. Liu, Electroanalysis, 2003, 15, 81-96.

126 D. Samanta and A. Sarkar, Chem. Soc. Rev., 2011, 40, 25672592.

127 A. Kumar, H. A. Biebuyck and G. M. Whitesides, Langmuir, 1994, 10, 1498-1511.

128 R. K. Smith, P. A. Lewis and P. S. Weiss, Prog. Surf. Sci., 2004, 75, 1-68.

129 M. Mrksich and G. M. Whitesides, Trends Biotechnol., 1995, 13, 228-235.

130 A. Y. Fadeev and T. J. McCarthy, Langmuir, 2000, 16, 72687274.

131 S.-J. Chun, S.-Y. Lee, G.-Y. Jeong and J. H. Kim, J. Ind. Eng. Chem., 2012, 18, 1122-1127.

132 B. D. Booth, S. G. Vilt, J. B. Lewis, J. L. Rivera, E. A. Buehler, C. McCabe and G. K. Jennings, Langmuir, 2011, 27, 59095917.

133 Y. Song, R. Premachandran Nair, M. Zou and Y. A. Wang, Thin Solid Films, 2010, 518, 3801-3807.

134 J. Cancino and S. A. S. Machado, Electrochim. Acta, 2012, 72, 108-113.

135 M. J. Wirth, R. W. P. Fairbank and H. O. Fatunmbi, Science, 1997, 275, 44-47.

136 J. Cancino, C. A. Razzino, V. Zucolotto and S. A. S. Machado, Electrochim. Acta, 2013, 87, 717-723.

137 T. Deng, J.-S. Li, S.-Y. Huan, H.-F. Yang, H. Wang, G.-L. Shen and R.-Q. Yu, Biosens. Bioelectron., 2006, 21, 1545-1552.

138 A. Brechling, M. Sundermann, U. Kleineberg and U. Heinzmann, Thin Solid Films, 2003, 433, 281-286.

139 M. V. Bracamonte, O. E. L. Pérez, M. L. Teijelo, G. A. Rivas and N. F. Ferreyra, Electrochim. Acta, 2014, 146, 178-185.

140 F. Bordi, C. Cametti and A. Gliozzi, Bioelectrochemistry, 2002, 57, 39-46.

141 H. T. Tien and A. L. Ottova, Colloids Surf., A, 1999, 149, 217233.

142 R. P. Richter, R. Bérat and A. R. Brisson, Langmuir, 2006, 22, 3497-3505.

143 S. Terrettaz, M. Mayer and H. Vogel, Langmuir, 2003, 19, 5567-5569. 
144 F. Berger, J. Delhalle and Z. Mekhalif, Appl. Surf. Sci., 2010, 256, 7131-7137.

145 Z. Zhu, G. Xu, Y. An and C. He, Colloids Surf., A, 2014, 457, 408-413.

146 K. Deng, R. J. Collins, M. Mehregany and C. N. Sukenik, J. Electrochem. Soc., 1995, 142, 1278-1285.

147 Y. Liu, P. Liu, Y. Xiao and J. Luo, Appl. Surf. Sci., 2012, 258, 8533-8537.

148 M. Cichomski, K. Kośla, J. Grobelny, W. Kozłowski and W. Szmaja, Appl. Surf. Sci., 2013, 273, 570-577.

149 L. Wang, R. J. K. Wood, T. J. Harvey, S. Morris, H. E. G. Powrie and I. Care, Wear, 2003, 255, 657-668.

150 F. E. Kennedy, Y. Lu and I. Baker, Tribol. Int., 2015, 82(Part B), 534-542.

151 R. Novak and T. Polcar, Tribol. Int., 2014, 74, 154-163.

152 D. L. Burris and W. G. Sawyer, Tribol. Lett., 2009, 35, 17-23.

153 T. L. Schmitz, J. E. Action, J. C. Ziegert and W. G. Sawyer, J. Tribol., 2005, 127, 673-678.

154 T. J. Kamps, J. C. Walker, R. J. Wood, P. M. Lee and A. G. Plint, Wear, 2015, 332-333, 1193-1199.

155 C. B. Mohan, C. Divakar, K. Venkatesh, K. Gopalakrishna, K. S. Mahesh Lohith and T. N. Naveen, Wear, 2009, 267, 1111-1116.

156 D. H. Hwang and K. H. Zum Gahr, Wear, 2003, 255, 365375.

157 M. Kosinskiy, S. I.-U. Ahmed, Y. Liu and J. A. Schaefer, Tribol. Int., 2012, 56, 81-88.

158 E.-S. Yoon, R. A. Singh, H.-J. Oh and H. Kong, Wear, 2005, 259, 1424-1431.

159 B. Bhushan and S. Sundararajan, Acta Mater., 1998, 46, 3793-3804.

160 S. P. Ho, R. W. Carpick, T. Boland and M. LaBerge, Wear, 2002, 253, 1145-1155.

161 B. Bhushan, Tribol. Int., 1995, 28, 85-96.

162 K.-H. Cha and D.-E. Kim, Wear, 2001, 251, 1169-1176.

163 V. DePalma and N. Tillman, Langmuir, 1989, 5, 868-872.

164 E. E. Flater, W. R. Ashurst and R. W. Carpick, Langmuir, 2007, 23, 9242-9252.

165 M. Garcia-Parajo, C. Longo, J. Servat, P. Gorostiza and F. Sanz, Langmuir, 1997, 13, 2333-2339.

166 R. A. Singh, J. Kim, S. W. Yang, J.-E. Oh and E.-S. Yoon, Wear, 2008, 265, 42-48.

167 S. Strobl, T. Lube, P. Supancic, M. Stoiser, O. Schöppl and R. Danzer, J. Eur. Ceram. Soc., 2014, 34, 4167-4176.

168 L. Wang, R. W. Snidle and L. Gu, Wear, 2000, 246, 159-173. 169 K. Thoma, L. Rohr, H. Rehmann, S. Roos and J. Michler, Tribol. Int., 2004, 37, 463-471.

170 S. A. Baker, M. J. Dellavecchia, B. W. Smith and J. D. Winefordner, Anal. Chim. Acta, 1997, 355, 113-119.

171 R. C. Dante and C. K. Kajdas, Wear, 2012, 288, 27-38.

172 B. S. Bal and M. N. Rahaman, Acta Biomater., 2012, 8, 28892898.

173 V. Ferreira, H. N. Yoshimura and A. Sinatora, Wear, 2012, 296, 656-659.

174 Y. Hibi and Y. Enomoto, Wear, 1989, 133, 133-145.

$175 \mathrm{~J} . \mathrm{Xu}$ and K. Kato, Wear, 2000, 245, 61-75.
176 S. Hampshire, Journal of Achievements of Materials and Manufacturing Engineering, 2007, 24, 43-50.

177 F. L. Riley, J. Am. Ceram. Soc., 2000, 83, 245-265.

178 H. Bhaskaran, B. Gotsmann, A. Sebastian, U. Drechsler, M. A. Lantz, M. Despont, P. Jaroenapibal, R. W. Carpick, Y. Chen and K. Sridharan, Nat. Nanotechnol., 2010, 5, 181-185.

179 R. S. Gates and S. M. Hsu, Tribol. Trans., 2000, 43, 269-274.

180 W. Hartung, A. Rossi, S. Lee and N. Spencer, Tribol. Lett., 2009, 34, 201-210.

181 H. K. Hunt and A. M. Armani, IEEE J. Sel. Top. Quantum Electron., 2014, 20, 121-133.

182 M. M. Sung, G. J. Kluth and R. Maboudian, J. Vac. Sci. Technol., A, 1999, 17, 540-544.

183 G. J. Kluth, M. M. Sung and R. Maboudian, Langmuir, 1997, 13, 3775-3780.

184 M. Kölbel, R. W. Tjerkstra, G. Kim, J. Brugger, C. J. M. van Rijn, W. Nijdam, J. Huskens and D. N. Reinhoudt, Adv. Funct. Mater., 2003, 13, 219-224.

185 J. Diao, D. Ren, J. R. Engstrom and K. H. Lee, Anal. Biochem., 2005, 343, 322-328.

186 R. Barattin and N. Voyer, Chem. Commun., 2008, 1513-1532.

187 V. V. Tsukruk and V. N. Bliznyuk, Langmuir, 1998, 14, 446455.

188 B. Daniel and H. Christofer, J. Micromech. Microeng., 2007, 17, 1326.

189 U. Zaghloul, G. Papaioannou, B. Bhushan, F. Coccetti, P. Pons and R. Plana, Microelectron. Reliab., 2011, 51, 1810-1818.

190 Z. Yapu, Acta Mech. Sin., 2003, 19, 1-10.

191 E. Kutnyanszky and G. J. Vancso, Eur. Polym. J., 2012, 48, 815.

192 T. Ito, M. Namba, P. Bühlmann and Y. Umezawa, Langmuir, 1997, 13, 4323-4332.

193 J. E. Headrick and C. L. Berrie, Langmuir, 2004, 20, 41244131.

194 C. D. Frisbie, L. F. Rozsnyai, A. Noy, M. S. Wrighton and C. M. Lieber, Science, 1994, 265, 2071-2074.

195 S. S. Kelkar, D. Chiavetta and C. A. Wolden, Appl. Surf. Sci., 2013, 282, 291-296.

196 D. Wang, Y. Ni, Q. Huo and D. E. Tallman, Thin Solid Films, 2005, 471, 177-185.

197 B. Panjwani and S. K. Sinha, J. Mech. Behav. Biomed. Mater., 2012, 15, 103-111.

198 P. F. Li, Y. Xu and X.-H. Cheng, Surf. Coat. Technol., 2013, 232, 331-339.

199 P. F. Li, H. Zhou and X.-H. Cheng, Appl. Surf. Sci., 2013, 285(Part B), 937-944.

200 S. Jadhav, Cent. Eur. J. Chem., 2011, 9, 369-378.

201 M. Ratoi, C. Bovington and H. Spikes, J. Jpn. Soc. Tribol., 2001, 1281-1286.

202 Y. Wan, Y. Wang, Q. Zhang, Z. Wang, Z. Xu, C. Liu and J. Zhang, Appl. Surf. Sci., 2012, 259, 147-152.

203 Q. Zhang, Y. Wan, Y. Li, S. Yang and W. Yao, Appl. Surf. Sci., 2013, 280, 545-549.

204 R. Simič and M. Kalin, Appl. Surf. Sci., 2013, 283, 460-470. 
205 O. Azzaroni, J. Polym. Sci., Part A: Polym. Chem., 2012, 50, 3225-3258.

206 Q. Wei, M. Cai, F. Zhou and W. Liu, Macromolecules, 2013, 46, 9368-9379.

207 J. Yan, B. Li, F. Zhou and W. Liu, ACS Macro Lett., 2013, 2, 592-596.

208 R. Bielecki, P. Doll and N. Spencer, Tribol. Lett., 2013, 49, 273-280.

209 T. Goren, N. D. Spencer and R. Crockett, RSC Adv., 2014, 4, 21497-21503.

210 S. Edmondson, V. L. Osborne and W. T. S. Huck, Chem. Soc. Rev., 2004, 33, 14-22.

211 B. Li, B. Yu, Q. Ye and F. Zhou, Acc. Chem. Res., 2015, 48, 229-237.

212 S. Edmondson and S. P. Armes, Polym. Int., 2009, 58, 307316.

213 S. Edmondson, C.-D. Vo, S. P. Armes and G.-F. Unali, Macromolecules, 2007, 40, 5271-5278.

214 S. Peng and B. Bhushan, RSC Adv., 2012, 2, 8557-8578.

215 F. Zhou and W. T. S. Huck, Phys. Chem. Chem. Phys., 2006, 8, 3815-3823.

216 C. Xu, T. Wu, J. D. Batteas, C. M. Drain, K. L. Beers and M. J. Fasolka, Appl. Surf. Sci., 2006, 252, 2529-2534.

217 C. Yoshikawa, J. Qiu, C.-F. Huang, Y. Shimizu, J. Suzuki and E. van den Bosch, Colloids Surf., B, 2015, 127, 213-220.

218 Y. Inoue and K. Ishihara, Colloids Surf., B, 2010, 81, 350357.

219 B. F. L. Lai, A. L. Creagh, J. Janzen, C. A. Haynes, D. E. Brooks and J. N. Kizhakkedathu, Biomaterials, 2010, 31, 6710-6718.

220 S. Yamamoto, M. Ejaz, Y. Tsujii and T. Fukuda, Macromolecules, 2000, 33, 5608-5612.

221 A. Olivier, F. Meyer, J.-M. Raquez, P. Damman and P. Dubois, Prog. Polym. Sci., 2012, 37, 157-181.

222 R. Barbey, L. Lavanant, D. Paripovic, N. Schüwer, C. Sugnaux, S. Tugulu and H.-A. Klok, Chem. Rev., 2009, 109, 5437-5527.

223 W. Hadasha and B. Klumperman, Polym. Int., 2014, 63, 824-834.

224 J. Pyun, T. Kowalewski and K. Matyjaszewski, Macromol. Rapid Commun., 2003, 24, 1043-1059.

225 R. Toomey and M. Tirrell, Annu. Rev. Phys. Chem., 2008, 59, 493-517.

226 A. T. Nguyen, J. Baggerman, J. M. J. Paulusse, C. J. M. van Rijn and H. Zuilhof, Langmuir, 2011, 27, 2587-2594.

227 C. Kang, R. M. Crockett and N. D. Spencer, Macromolecules, 2014, 47, 269-275.

228 O. Nuyken and S. Pask, Polymer, 2013, 5, 361.

229 S. Penczek and G. Moad, Pure Appl. Chem., 2008, vol. 80, 2163.

230 K. Udipi, R. S. Davé, R. L. Kruse and L. R. Stebbins, Polymer, 1997, 38, 927-938.

231 C. W. Bielawski and R. H. Grubbs, Prog. Polym. Sci., 2007, 32, 1-29.

232 C. J. Hawker, J. Am. Chem. Soc., 1994, 116, 11185-11186.

233 D. Benoit, V. Chaplinski, R. Braslau and C. J. Hawker, J. Am. Chem. Soc., 1999, 121, 3904-3920.
234 M. Semsarilar and S. Perrier, Nat. Chem., 2010, 2, 811-820. 235 J. Chiefari, Y. K. Chong, F. Ercole, J. Krstina, J. Jeffery, T. P. T. le, R. T. A. Mayadunne, G. F. Meijs, C. L. Moad, G. Moad, E. Rizzardo and S. H. Thang, Macromolecules, 1998, 31, 5559-5562.

236 G. Moad, E. Rizzardo and S. H. Thang, Aust. J. Chem., 2005, 58, 379-410.

237 B. Giechaskiel, M. Maricq, L. Ntziachristos, C. Dardiotis, X. Wang, H. Axmann, A. Bergmann and W. Schindler, J. Aerosol Sci., 2014, 67, 48-86.

238 J. Yan, B. Li, B. Yu, W. T. S. Huck, W. Liu and F. Zhou, Angew. Chem., Int. Ed., 2013, 52, 9125-9129.

239 M. Kato, M. Kamigaito, M. Sawamoto and T. Higashimura, Macromolecules, 1995, 28, 1721-1723.

240 J.-S. Wang and K. Matyjaszewski, J. Am. Chem. Soc., 1995, 117, 5614-5615.

241 P. Król and P. Chmielarz, Prog. Org. Coat., 2014, 77, 913948.

242 H. Liu, C. T. O'Mahony, F. Audouin, C. Ventura, M. Morris and A. Heise, Macromol. Chem. Phys., 2012, 213, 108-115.

243 W. Song, C. Xiao, L. Cui, Z. Tang, X. Zhuang and X. Chen, Colloids Surf., B, 2012, 93, 188-194.

244 X. Huang and M. J. Wirth, Anal. Chem., 1997, 69, 45774580.

245 M. Ejaz, S. Yamamoto, K. Ohno, Y. Tsujii and T. Fukuda, Macromolecules, 1998, 31, 5934-5936.

246 E. Turan, S. Demirci and T. Caykara, Thin Solid Films, 2010, 518, 5950-5954.

247 H. Fischer, Chem. Rev., 2001, 101, 3581-3610.

248 H. Gao and K. Matyjaszewski, Prog. Polym. Sci., 2009, 34, 317-350.

249 K. Matyjaszewski, Macromolecules, 2012, 45, 4015-4039.

250 L. J. T. Landherr, C. Cohen, P. Agarwal and L. A. Archer, Langmuir, 2011, 27, 9387-9395.

251 K. Ohno, T. Morinaga, K. Koh, Y. Tsujii and T. Fukuda, Macromolecules, 2005, 38, 2137-2142.

252 K. Matyjaszewski, P. J. Miller, N. Shukla, B. Immaraporn, A. Gelman, B. B. Luokala, T. M. Siclovan, G. Kickelbick, T. Vallant, H. Hoffmann and T. Pakula, Macromolecules, 1999, 32, 8716-8724.

253 M. Husseman, E. E. Malmström, M. McNamara, M. Mate, D. Mecerreyes, D. G. Benoit, J. L. Hedrick, P. Mansky, E. Huang, T. P. Russell and C. J. Hawker, Macromolecules, 1999, 32, 1424-1431.

254 B. D. Karstedt, US Pat., US3775452, 1971.

255 W. Zhou, H. Liu, H. Ye, H. Cui, R. Wang, J. Li and X. Zhang, Powder Technol., 2013, 249, 1-6.

256 W. Feng, J. L. Brash and S. Zhu, Biomaterials, 2006, 27, 847855.

257 D. J. Siegwart, J. K. Oh and K. Matyjaszewski, Prog. Polym. Sci., 2012, 37, 18-37.

258 K. Matyjaszewski, H. Dong, W. Jakubowski, J. Pietrasik and A. Kusumo, Langmuir, 2007, 23, 4528-4531.

259 S. Munirasu, R. G. Karunakaran, J. Rühe and R. Dhamodharan, Langmuir, 2011, 27, 13284-13292.

260 E. Turan and T. Caykara, React. Funct. Polym., 2011, 71, 1089-1095. 
261 E. Öztürk, E. Turan and T. Caykara, Appl. Surf. Sci., 2010, 257, 1015-1020.

262 A. Nomura, K. Okayasu, K. Ohno, T. Fukuda and Y. Tsujii, Macromolecules, 2011, 44, 5013-5019.

263 S. Yamamoto, M. Ejaz, Y. Tsujii, M. Matsumoto and T. Fukuda, Macromolecules, 2000, 33, 5602-5607.

264 D. A. Shipp and K. Matyjaszewski, Macromolecules, 2000, 33, 1553-1559.

265 K. L. Beers, S. G. Gaynor, K. Matyjaszewski, S. S. Sheiko and M. Möller, Macromolecules, 1998, 31, 9413-9415.

266 T. Pintauer and K. Matyjaszewski, Coord. Chem. Rev., 2005, 249, 1155-1184.

267 B. Zhu and S. Edmondson, Polymer, 2011, 52, 2141-2149.

268 F. Jiang, W. H. Meyer and J. Zhang, Colloids Surf., A, 2013, 436, 302-308.

269 Y. Liu, Y. Xiao and J. Luo, Sci. China: Technol. Sci., 2012, 55, 3352-3358.

270 A. M. Elsen, J. Burdyńska, S. Park and K. Matyjaszewski, ACS Macro Lett., 2013, 2, 822-825.

271 J. Ran, L. Wu, Z. Zhang and T. Xu, Prog. Polym. Sci., 2014, 39, 124-144.

272 N. V. Tsarevsky and K. Matyjaszewski, Chem. Rev., 2007, 107, 2270-2299.

273 K. Min, H. Gao and K. Matyjaszewski, Macromolecules, 2007, 40, 1789-1791.

274 W. Jakubowski and K. Matyjaszewski, Macromolecules, 2005, 38, 4139-4146.

275 T. Pintauer and K. Matyjaszewski, Chem. Soc. Rev., 2008, 37, 1087-1097.

276 C. Sun, F. Zhou, L. Shi, B. Yu, P. Gao, J. Zhang and W. Liu, Appl. Surf. Sci., 2006, 253, 1729-1735.

277 F. Seeliger and K. Matyjaszewski, Macromolecules, 2009, 42, 6050-6055.
278 J. Klein, E. Kumacheva, D. Mahalu, D. Perahia and L. J. Fetters, Nature, 1994, 370, 634-636.

279 G. Fontani, R. Gaspari, N. D. Spencer, D. Passerone and R. Crockett, Langmuir, 2013, 29, 4760-4771.

280 R. Heeb, R. M. Bielecki, S. Lee and N. D. Spencer, Macromolecules, 2009, 42, 9124-9132.

281 H. Sakata, M. Kobayashi, H. Otsuka and A. Takahara, Polym. J., 2005, 37, 767-775.

282 D. Irfachsyad, D. Tildesley and P. Malfreyt, Phys. Chem. Chem. Phys., 2002, 4, 3008-3015.

283 O. Tairy, N. Kampf, M. J. Driver, S. P. Armes and J. Klein, Macromolecules, 2015, 48, 140-151.

284 N. S. Bhairamadgi, S. P. Pujari, F. A. M. Leermakers, C. J. M. van Rijn and H. Zuilhof, Langmuir, 2014, 30, 2068-2076.

285 M. Kobayashi, Y. Terayama, N. Hosaka, M. Kaido, A. Suzuki, N. Yamada, N. Torikai, K. Ishihara and A. Takahara, Soft Matter, 2007, 3, 740-746.

286 L. C. H. Moh, M. D. Losego and P. V. Braun, Langmuir, 2011, 27, 3698-3702.

287 F. T. Limpoco, R. C. Advincula and S. S. Perry, Langmuir, 2007, 23, 12196-12201.

288 G. W. de Groot, M. G. Santonicola, K. Sugihara, T. Zambelli, E. Reimhult, J. Vörös and G. J. Vancso, ACS Appl. Mater. Interfaces, 2013, 5, 1400-1407.

289 A. T. Nguyen, J. Baggerman, J. M. J. Paulusse, H. Zuilhof and C. J. M. van Rijn, Langmuir, 2012, 28, 604-610.

290 S. Gabriel, C. Jérôme, R. Jérôme, C.-A. Fustin, A. Pallandre, J. Plain, A. M. Jonas and A.-S. Duwez, J. Am. Chem. Soc., 2007, 129, 8410-8411.

291 R. Gong, S. Maclaughlin and S. Zhu, Appl. Surf. Sci., 2008, 254, 6802-6809. 